TRANSACTIONS OF THE

AMERICAN MATHEMATICAL SOCIETY

Volume 362, Number 6, June 2010, Pages 3027-3067

S 0002-9947(09)04933-2

Article electronically published on December 22, 2009

\title{
MODULAR INVARIANCE FOR CONFORMAL FULL FIELD ALGEBRAS
}

\author{
YI-ZHI HUANG AND LIANG KONG
}

\begin{abstract}
Let $V^{L}$ and $V^{R}$ be simple vertex operator algebras satisfying certain natural uniqueness-of-vacuum, complete reducibility and cofiniteness conditions and let $F$ be a conformal full field algebra over $V^{L} \otimes V^{R}$. We prove that the $q_{\tau}-\overline{q_{\tau}}$-traces (natural traces involving $q_{\tau}=e^{2 \pi i \tau}$ and $\overline{q_{\tau}}=\overline{e^{2 \pi i \tau}}$ ) of geometrically modified genus-zero correlation functions for $F$ are convergent in suitable regions and can be extended to doubly periodic functions with periods 1 and $\tau$. We obtain necessary and sufficient conditions for these functions to be modular invariant. In the case that $V^{L}=V^{R}$ and $F$ is one of those constructed by the authors in an earlier paper, we prove that all these functions are modular invariant.
\end{abstract}

\section{INTRODUCTION}

In this paper, we construct genus-one full conformal field theories (genus-one conformal field theories with both chiral and antichiral parts) from genus-zero full conformal field theories constructed and studied in [HK]. More precisely, we construct genus-one correlation functions from genus-zero correlation functions for a conformal full field algebra over $V^{L} \otimes V^{R}$ in the sense of [HK], where $V^{L}$ and $V^{R}$ are vertex operator algebras satisfying certain natural uniqueness-of-vacuum, complete reducibility and cofiniteness conditions (see below).

Since Kontsevich and Segal S1, S2, S3 (cf. Kont) gave a geometric definition of (two-dimensional) conformal field theory in 1987 by axiomatizing the properties of path integrals used by physicists, constructing conformal field theories satisfying this definition became an important unsolved mathematical problem. In this definition, just as in its original physical form formulated by Belavin, Polyakov and Zamolodchikov [BPZ], conformal field theories have both chiral and antichiral parts.

Around the same time, E. Verlinde [V] and Moore-Seiberg [MS1] MS2] (see also [MS3]) made a major breakthrough by discovering the relation between fusion rules and modular transformations and the modular tensor category structures associated to rational conformal field theories. These fundamental works are based on the existence of such full conformal field theories with both chiral and antichiral parts.

In mathematics, however, it is mostly chiral conformal field theories that are constructed and studied. To describe chiral conformal field theories, Segal [S2],

Received by the editors March 10, 2008.

2000 Mathematics Subject Classification. Primary 17B69; Secondary 81T40.

(C)2009 American Mathematical Society

Reverts to public domain 28 years from publication 3027 
S3] introduced modular functors and weakly conformal field theories. For a full conformal field theory, its chiral and antichiral parts are both weakly conformal field theories and each of these weakly conformal field theories have modular functors. The problem of constructing full conformal field theories can then be divided into three steps: (i) Construct modular functors. (ii) Construct the weakly conformal field theories over these modular functors. (iii) Construct full conformal field theories from two compatible weakly conformal field theories.

For some of the applications of the ideas coming from conformal field theory (for example, for the construction and study of knot and three-manifold invariants and the proof of the Verlinde formula in algebraic geometry), a construction of modular functors is already enough. See, for example, $[\mathrm{Tu}]$ and $[\mathrm{BK}]$ for the construction of some examples of modular functors. In [H11] (see also [H8 for an announcement and [H9] for an exposition), the first author constructed modular tensor categories from representations of vertex operator algebras satisfying the conditions mentioned above. Combined with the results in [Tu] and $[\mathrm{BK}$, this result of [H11] in fact gives a construction of modular functors from representations of vertex operator algebras satisfying the conditions mentioned above.

However, for some other applications, modular functors are far from enough. One extreme example is the conformal-field-theoretic construction used in FrenkelLepowsky-Meurman's proof of the McKay-Thompson conjecture on the existence of the moonshine module [FLM] and in Borcherds' proof of the Monstrous moonshine conjecture $[\mathrm{B}$. In this example, the modular functor associated to the moonshine module vertex operator algebra is actually trivial and thus does not play any role. On the other hand, we know that the construction of the moonshine module vertex operator algebra by Frenkel-Lepowsky-Meurman and the proof of the Monstrous moonshine conjecture by Borcherds can be interpreted as one of the deepest applications of conformal field theory. See also the recent work [W] of Witten for the possible important role of this vertex operator algebra in the physics of threedimensional gravity.

A program to construct conformal field theories was first initiated by I. Frenkel even before Kontsevich and Segal gave their geometric definition of conformal field theory. Under the direction of I. Frenkel, Tsukada [S constructed genus-zero and genus-one parts of conformal field theories associated to tori. He constructed not only chiral theories but also full theories which contain both chiral and antichiral parts. In [KO], Kapustin and Orlov studied genus-zero full superconformal field theories associated to tori in connection with mirror symmetry and $D$-branes, but the modular invariance of such theories was not discussed in that paper.

In [H1], [H2], [H3], [H4], [H5], [H6] and [H7, the first author constructed chiral genus-zero and genus-one conformal field theories from representations of vertex operator algebras satisfying suitable conditions. In [HK, the authors constructed genus-zero full conformal field theories from representations of vertex operator algebras satisfying the conditions mentioned above and given precisely below and in Section 3 (see also Kong1 and Kong2]). The next natural but crucial step in this program of constructing conformal field theories from representations of vertex operator algebras is to construct the genus-one full conformal field theories.

The purpose of the present paper is to carry out this crucial step. More precisely, let $V^{L}$ and $V^{R}$ be simple vertex operator algebras satisfying the following conditions for a vertex operator algebra $V$ : (i) For $n<0, V_{(n)}=0, V_{(0)}=\mathbb{C} 1$ and $W_{(0)}=0$ 
for an irreducible $V$-module $W$ not isomorphic to $V$. (ii) Every $\mathbb{N}$-gradable weak $V$-module is completely reducible. (iii) $V$ is $C_{2}$-cofinite; that is, $\operatorname{dim} V / C_{2}(V)<\infty$, where $C_{2}(V)$ is the subspace of $V$ spanned by elements of the form $u_{-2} v$ for $u, v \in V$. Let $F$ be a conformal full field algebra over $V^{L} \otimes V^{R}$ (see [HK] or Section 1 for the definition and basic properties). We construct genus-one correlation functions using $q_{\tau}-\overline{q_{\tau}}$-traces (natural traces involving $q_{\tau}=e^{2 \pi i \tau}$ and $\overline{q_{\tau}}=e^{2 \pi i \tau}$ ) of geometrically modified genus-zero correlation functions for $F$. We prove that these functions are doubly periodic and we obtain conditions which are equivalent to the modular invariance of these functions. When $V^{L}=V^{R}=V$ and $F$ is the conformal full field algebra over $V \otimes V$ constructed by the authors in $[\mathrm{HK}$, we prove that these conditions are satisfied and thus all these functions are modular invariant.

We note that based on the existence of the structure of a modular tensor category on the category of modules for a vertex operator algebra, the existence of conformal blocks with monodromies compatible with the modular tensor category and all the necessary convergence properties (in particular, the corresponding conformal field theories must be "rational"), Felder, Fröhlich, Fuchs and Schweigert FFFS, Fuchs, Runkel, Schweigert and Fjelstad [FRS1, FRS2, [FRS3, FRS4, [FRS5, FjFRS1, FjFRS2, and Fröhlich, Fuchs, Runkel and Schweigert FrFRS studied open-closed conformal field theories (in particular full (closed) conformal field theories) using the theory of tensor categories and three-dimensional topological field theories. In particular, they constructed correlation functions as states in some three-dimensional topological field theories. In $[\mathrm{HK}$ and the present paper, what we need in our work are theorems proved by the first author in [H6], [H7], [H10 and H11] when the vertex operator algebras we start with satisfy some natural conditions. Our work in $[\mathrm{HK}]$ and the present paper not only replaced these fundamental but hard-to-verify assumptions (in particular, the assumption that the corresponding conformal field theories are "rational") by natural, purely algebraic and easy-to-verify conditions on vertex operator algebras, but also constructed explicitly genus-zero and genus-one correlation functions from intertwining operators for the vertex operator algebras.

The present paper depends heavily on the results obtained in [H7, even more heavily than the papers [H10 and [H11. In [H10 and [H11, we need only the properties of certain special two-point genus-one chiral correlation functions obtained in [H7], namely, those obtained from iterates of intertwining operators with the intermediate modules being the vertex operator algebra itself. In this paper, we need the full strength of the results obtained in [H7. In particular, using the results of [H7], we prove a symmetry property of the matrix elements of the actions of the modular transformation $\tau \mapsto-\frac{1}{\tau}$ on the space of one-point genus-one correlation functions (Theorem 4.13), which is a generalization of the symmetry property of the matrix elements of the action of the same modular transformation on the space of vacuum characters (Theorem 5.6 in [H10]). This generalization is exactly what we need in Section 4 to prove the modular invariance of the genus-one correlation functions for the conformal full field algebras constructed in [HK]. To prove Theorem 4.13, we prove an explicit formula (4.24) for these matrix elements. In the case that $a=e$, we recover the formula for the matrix elements of the action of the same modular transformation on the space of vacuum characters, obtained first by Moore-Seiberg from the Verlinde formula and proved for vertex operator algebras satisfying the conditions above by the first author in [H7]. Note that in 
the case $a=e$ this formula (4.24) was shown in H11 to be equivalent to the nondegeneracy property of the semisimple ribbon (tensor) category of modules for the vertex operator algebra. In the special case that fusion rules are all equal to 0 or 1 (for example, in the case of discrete series), a formula for these matrix elements was given in MS3.

The present paper is organized as follows: In Section 1, we recall the basic definition and constructions in the theory of conformal full field algebras over $V^{L} \otimes V^{R}$ given first in $\mathrm{HK}$. In Section 2, we recall the chiral genus-one theory constructed from intertwining operator algebras in [H7]. These two sections are given here for the convenience of the reader. The reader is referred to $[\mathrm{HK}]$ and [H7] for more details. In Section 3, we prove the convergence of $q_{\tau}-\overline{q_{\tau}}$-traces of genus-zero correlation functions in suitable regions and show that these can be extended to doubly periodic functions with periods 1 and $\tau$. We also give conditions which are equivalent to the modular invariance of these functions in this section. In Section 4, we study the matrix elements of the action of the modular transformation $S: \tau \mapsto-\frac{1}{\tau}$ on chiral genus-one correlation functions. In particular we obtain an explicit formula for these matrix elements. This formula allows us to derive a symmetry property of these matrix elements. Using these results and the results obtained in Section 3, we prove the modular invariance of the correlation functions for the conformal full field algebras constructed in [HK].

\section{Conformal full field algebras}

In this section, we recall the notion of conformal full field algebra over $V^{L} \otimes V^{R}$ introduced in $[\mathrm{HK}$ and review the construction of conformal full field algebras over $V^{L} \otimes V^{R}$ when $V^{L}=V^{R}$ satisfying suitable conditions given in the same paper. See [HK] for more details and other variants of full field algebras.

Let $\mathbb{F}_{n}(\mathbb{C})=\left\{\left(z_{1}, \ldots, z_{n}\right) \in \mathbb{C}^{n} \mid z_{i} \neq z_{j}\right.$ if $\left.i \neq j\right\}$. For an $\mathbb{R} \times \mathbb{R}$-graded vector space $F=\bigsqcup_{r, s \in \mathbb{R}} F_{(r, s)}$, let $F^{\prime}=\bigsqcup_{r, s \in \mathbb{R}} F_{(r, s)}^{*}$ and $\bar{F}=\prod_{r, s \in \mathbb{R}} F_{(r, s)}$ be the graded dual and algebraic completion of $F$, respectively. For $r, s \in \mathbb{R}$, let $P_{r, s}$ be the projection from $F$ or $\bar{F}$ to $F_{(r, s)}$. A series $\sum f_{n}$ in $\bar{F}$ is said to be absolutely convergent if for any $f^{\prime} \in F^{\prime}, \sum\left|\left\langle f^{\prime}, f_{n}\right\rangle\right|$ is convergent. The sums $\sum\left|\left\langle f^{\prime}, f_{n}\right\rangle\right|$ for $f^{\prime} \in F^{\prime}$ define a linear functional on $F^{\prime}$. We call this linear functional the sum of the series and denote it by the same notation $\sum f_{n}$. If the homogeneous subspaces of $F$ are all finite-dimensional, then $\bar{F}=\left(F^{\prime}\right)^{*}$ and, in this case, the sum of an absolutely convergent series is always in $\bar{F}$. When the sum is in $\bar{F}$, we say that the series is absolutely convergent in $\bar{F}$.

Definition 1.1. Let $\left(V^{L}, Y^{L}, \mathbf{1}^{L}, \omega^{L}\right)$ and $\left(V^{R}, Y^{R}, \mathbf{1}^{R}, \omega^{R}\right)$ be vertex operator algebras. A conformal full field algebra over $V^{L} \otimes V^{R}$ is an $\mathbb{R} \times \mathbb{R}$-graded vector space $F=\bigsqcup_{r, s \in \mathbb{R}} F_{(r, s)}$ (graded by left conformal weight or simply left weight and by right conformal weight or simply right weight), equipped with correlation function maps

$$
\begin{aligned}
m_{n}: \quad F^{\otimes n} \times \mathbb{F}_{n}(\mathbb{C}) & \rightarrow \bar{F} \\
\left(u_{1} \otimes \cdots \otimes u_{n},\left(z_{1}, \ldots, z_{n}\right)\right) & \mapsto m_{n}\left(u_{1}, \ldots, u_{n} ; z_{1}, \bar{z}_{1}, \ldots, z_{n}, \bar{z}_{n}\right),
\end{aligned}
$$

for $n \in \mathbb{Z}_{+}$, an injective grading-preserving linear map $\rho$ from $V^{L} \otimes V^{R}$ to $F$ 
satisfying the following axioms:

(1) There exists $M \in \mathbb{R}$ such that $F_{(r, s)}=0$ if $n<M$ or $m<M$.

(2) $\operatorname{dim} F_{(r, s)}<\infty$ for $m, n \in \mathbb{R}$.

(3) For $n \in \mathbb{Z}_{+}, m_{n}\left(u_{1}, \ldots, u_{n} ; z_{1}, \bar{z}_{1}, \ldots, z_{n}, \bar{z}_{n}\right)$ is linear in $u_{1}, \ldots, u_{n}$ and smooth in the real and imaginary parts of $z_{1}, \ldots, z_{n}$.

(4) For $u \in F, m_{1}(u ; 0,0)=u$.

(5) For $n \in \mathbb{Z}_{+}, u_{1}, \ldots, u_{n} \in F$,

$$
\begin{gathered}
m_{n+1}\left(u_{1}, \ldots, u_{n}, \mathbf{1} ; z_{1}, \bar{z}_{1}, \ldots, z_{n}, \bar{z}_{n}, z_{n+1}, \bar{z}_{n+1}\right) \\
=m_{n}\left(u_{1}, \ldots, u_{n} ; z_{1}, \bar{z}_{1}, \ldots, z_{n}, \bar{z}_{n}\right),
\end{gathered}
$$

where $\mathbf{1}=\rho\left(\mathbf{1}^{L} \otimes \mathbf{1}^{R}\right)$.

(6) The convergence property: For $k, l_{1}, \ldots, l_{k} \in \mathbb{Z}_{+}$and $u_{1}^{(1)}, \ldots, u_{l_{1}}^{(1)}, \ldots, u_{1}^{(k)}$, $\ldots, u_{l_{k}}^{(k)} \in F$, the series

$$
\begin{aligned}
& \sum_{r_{1}, s_{1}, \ldots, r_{k}, s_{k}} m_{k}\left(P_{r_{1}, s_{1}} m_{l_{1}}\left(u_{1}^{(1)}, \ldots, u_{l_{1}}^{(1)} ; z_{1}^{(1)}, \bar{z}_{1}^{(1)}, \ldots, z_{l_{1}}^{(1)}, \bar{z}_{l_{1}}^{(1)}\right), \ldots,\right. \\
& \left.P_{r_{k}, s_{k}} m_{l_{k}}\left(u_{1}^{(k)}, \ldots, u_{l_{k}}^{(k)} ; z_{1}^{(k)}, \bar{z}_{1}^{(k)}, \ldots, z_{l_{k}}^{(k)}, \bar{z}_{l_{k}}^{(k)}\right) ; z_{1}^{(0)}, \bar{z}_{1}^{(0)}, \ldots, z_{k}^{(0)}, \bar{z}_{k}^{(0)}\right)
\end{aligned}
$$

converges absolutely to

$$
\begin{aligned}
& m_{l_{1}+\cdots+l_{k}}\left(u_{1}^{(1)}, \ldots, u_{l_{k}}^{(k)} ; z_{1}^{(1)}+z_{1}^{(0)}, \bar{z}_{1}^{(1)}+\bar{z}_{1}^{(0)}, \ldots, z_{l_{1}}^{(1)}+z_{1}^{(0)}\right. \\
& \left.\bar{z}_{l_{1}}^{(1)}+\bar{z}_{1}^{(0)}, \ldots, z_{1}^{(k)}+z_{k}^{(0)}, \bar{z}_{1}^{(k)}+\bar{z}_{k}^{(0)}, \ldots, z_{l_{k}}^{(k)}+z_{k}^{(0)}, \bar{z}_{l_{k}}^{(k)}+\bar{z}_{k}^{(0)}\right)
\end{aligned}
$$

when $\left|z_{p}^{(i)}\right|+\left|z_{q}^{(j)}\right|<\left|z_{i}^{(0)}-z_{j}^{(0)}\right|$ for $i, j=1, \ldots, k, i \neq j$ and for $p=1, \ldots, l_{i}$ and $q=1, \ldots, l_{j}$.

(7) The permutation property: For any $n \in \mathbb{Z}_{+}$and any $\sigma \in S_{n}$, we have

$$
\begin{aligned}
& m_{n}\left(u_{1}, \ldots, u_{n} ; z_{1}, \bar{z}_{1}, \ldots, z_{n}, \bar{z}_{n}\right) \\
& \quad=m_{n}\left(u_{\sigma(1)}, \ldots, u_{\sigma(n)} ; z_{\sigma(1)}, \bar{z}_{\sigma(1)}, \ldots, z_{\sigma(n)}, \bar{z}_{\sigma(n)}\right)
\end{aligned}
$$

for $u_{1}, \ldots, u_{n} \in F$ and $\left(z_{1}, \ldots, z_{n}\right) \in \mathbb{F}_{n}(\mathbb{C})$.

(8) Let

$$
\mathbb{Y}: \begin{aligned}
F^{\otimes 2} \times \mathbb{C}^{\times} & \rightarrow \bar{F} \\
(u \otimes v, z, \bar{z}) & \mapsto \mathbb{Y}(u ; z, \bar{z}) v
\end{aligned}
$$

be given by

$$
\mathbb{Y}(u ; z, \bar{z}) v=m_{2}(u \otimes v ; z, \bar{z}, 0,0)
$$

for $u, v \in F$. Then

$$
\mathbb{Y}\left(\rho\left(u^{L} \otimes u^{R}\right) ; z, \bar{z}\right) \rho\left(v^{L} \otimes v^{R}\right)=\rho\left(Y^{L}\left(u^{L}, z\right) u^{R} \otimes Y^{R}\left(u^{R}, \bar{z}\right) v^{R}\right)
$$

for $u^{L}, v^{L} \in V^{L}, u^{R}, v^{R} \in V^{R}$, and there exist operators $L^{L}(n)$ and $L^{R}(n)$ for $n \in \mathbb{Z}$ such that

$$
\begin{aligned}
& \mathbb{Y}\left(\rho\left(\omega^{L} \otimes \mathbf{1}^{R}\right) ; z, \bar{z}\right)=\sum_{n \in \mathbb{Z}} L^{L}(n) z^{-n-2}, \\
& \mathbb{Y}\left(\rho\left(\mathbf{1}^{L} \otimes \omega^{R}\right) ; z, \bar{z}\right)=\sum_{n \in \mathbb{Z}} L^{R}(n) \bar{z}^{-n-2} .
\end{aligned}
$$

(9) The single-valuedness property: $e^{2 \pi i\left(L^{L}(0)-L^{R}(0)\right)}=I_{F}$. 
(10) The $L^{L}(0)$ - and $L^{R}(0)$-grading properties: For $r, s \in \mathbb{R}$ and $u \in F_{(r, s)}$,

$$
\begin{aligned}
L^{L}(0) u & =r u, \\
L^{R}(0) u & =s u,
\end{aligned}
$$

(11) The $L^{L}(0)$ - and $L^{R}(0)$-bracket properties: For $u \in F$,

$$
\begin{aligned}
{\left[L^{L}(0), \mathbb{Y}(u ; z, \bar{z})\right] } & =z \frac{\partial}{\partial z} \mathbb{Y}(u ; z, \bar{z})+\mathbb{Y}\left(L^{L}(0) u ; z, \bar{z}\right), \\
{\left[L^{R}(0), \mathbb{Y}(u ; z, \bar{z})\right] } & =\bar{z} \frac{\partial}{\partial \bar{z}} \mathbb{Y}(u ; z, \bar{z})+\mathbb{Y}\left(L^{R}(0) u ; z, \bar{z}\right) .
\end{aligned}
$$

(12) The $L^{L}(-1)$ - and $L^{R}(-1)$-derivative property: For $u \in F$,

$$
\begin{aligned}
& {\left[L^{L}(-1), \mathbb{Y}(u ; z, \bar{z})\right]=\mathbb{Y}\left(L^{L}(-1) u ; z, \bar{z}\right)=\frac{\partial}{\partial z} \mathbb{Y}(u ; z, \bar{z}),} \\
& {\left[L^{R}(-1), \mathbb{Y}(u ; z, \bar{z})\right]=\mathbb{Y}\left(L^{R}(-1) u ; z, \bar{z}\right)=\frac{\partial}{\partial \bar{z}} \mathbb{Y}(u ; z, \bar{z}) .}
\end{aligned}
$$

We denote the conformal full field algebra over $V^{L} \otimes V^{R}$ defined above by $(F, m, \rho)$ or simply by $F$. In the definition above, we use the notation

$$
m_{n}\left(u_{1}, \ldots, u_{n} ; z_{1}, \bar{z}_{1} \ldots, z_{n}, \bar{z}_{n}\right)
$$

instead of

$$
m_{n}\left(u_{1}, \ldots, u_{n} ; z_{1}, \ldots, z_{n}\right)
$$

to emphasize that these are in general not holomorphic in $z_{1}, \ldots, z_{n}$. For $u^{\prime} \in F^{\prime}$, $u_{1}, \ldots, u_{n} \in F$,

$$
\left\langle u^{\prime}, m_{n}\left(u_{1}, \ldots, u_{n} ; z_{1}, \bar{z}_{1} \ldots, z_{n}, \bar{z}_{n}\right)\right\rangle
$$

as a function of $z_{1}, \ldots, z_{n}$ is called a correlation function. The map $\mathbb{Y}$ is called the full vertex operator map and for $u \in F, \mathbb{Y}(u ; z, \bar{z})$ is called the full vertex operator associated to $u$. The element $\rho(\mathbf{1} \otimes \mathbf{1})$ is called the vacuum of $F$. The elements $\rho\left(\omega^{L} \otimes \mathbf{1}^{R}\right)$ and $\rho\left(\mathbf{1}^{L} \otimes \omega^{R}\right)$ are called the left conformal element and the right conformal element, respectively.

Homomorphisms and isomorphisms for conformal full field algebras over $V^{L} \otimes V^{R}$ are defined in the obvious way.

For a conformal full field algebra over $V^{L} \otimes V^{R}$, a formal full vertex operator map

$$
\begin{aligned}
\mathbb{Y}_{f}: F \otimes F & \rightarrow F\{x, \bar{x}\} \\
u \otimes v & \mapsto \mathcal{Y}_{f}(u ; x, \bar{x}) v
\end{aligned}
$$

was obtained in $\mathrm{HK}$ such that

$$
\mathbb{Y}(u ; z, \bar{z})=\left.\mathbb{Y}_{f}(u ; x, \bar{x})\right|_{x^{r}=e^{r \log z}, \bar{x}^{s}=e^{s \overline{\log z}}, r, s \in \mathbb{R}}
$$

for $u \in F$ and $z \in \mathbb{C}^{\times}$. We can also substitute $e^{r \log z}$ and $e^{s \overline{\log \zeta}}$ for $x^{r}$ and $\bar{x}^{s}$ to obtain $\mathbb{Y}(u ; z, \zeta)$ for $u \in F$.

For the operators $L^{L}(n)$ and $L^{R}(n)$ for $n \in \mathbb{Z}$, we have the following bracket formulas: For $m, n \in \mathbb{Z}$,

$$
\begin{aligned}
& {\left[L^{L}(m), L^{L}(n)\right]=(m-n) L^{L}(m+n)+\frac{c^{L}}{12}\left(m^{3}-m\right) \delta_{m+n, 0},} \\
& {\left[L^{R}(m), L^{R}(n)\right]=(m-n) L^{R}(m+n)+\frac{c^{R}}{12}\left(m^{3}-m\right) \delta_{m+n, 0},} \\
& {\left[L^{L}(m), L^{R}(n)\right]=0 .}
\end{aligned}
$$


Let $F$ be a module for the vertex operator algebra $V^{L} \otimes V^{R}$ and $\mathcal{Y}$ an intertwining operator of type $\left(\begin{array}{c}F \\ F F\end{array}\right)$. In [HK], a splitting $\mathbb{Y}^{\mathcal{Y}}:(F \otimes F) \times \mathbb{C}^{\times} \rightarrow \bar{F}$ and a formal splitting $\mathbb{Y}_{f}^{\mathcal{Y}}: F \otimes F \rightarrow \bar{F}\{x, \bar{x}\}$ of $\mathcal{Y}$ are constructed. Substituting $e^{r \log z}$ and $e^{s \overline{\log \zeta}}$ for $x^{r}$ and $\bar{x}^{s}$ in the images of $F \otimes F$ under $\mathbb{Y}_{f}^{\mathcal{Y}}$, we obtain an analytic splitting $\mathbb{Y}_{\text {an }}^{\mathcal{Y}}:(F \otimes F) \times\left(\mathbb{C}^{\times} \times \mathbb{C}^{\times}\right) \rightarrow \bar{F}$.

One of the main results of $[\mathrm{HK}]$ is the following theorem:

Theorem 1.2. Let $V^{L}$ and $V^{R}$ be vertex operator algebras satisfying the following conditions for a vertex operator algebra $V$ : (i) Every $\mathbb{C}$-graded $L(0)$-semisimple generalized $V$-module is a direct sum of $\mathbb{C}$-graded irreducible $V$-modules. (ii) There are only finitely many inequivalent $\mathbb{C}$-graded irreducible $V$-modules and they are all $\mathbb{R}$-graded. (iii) Every $\mathbb{R}$-graded irreducible $V$-module $W$ satisfies the $C_{1}$-cofiniteness condition, that is, $\operatorname{dim} W / C_{1}(W)<\infty$, where $C_{1}(V)$ is the subspace of $V$ spanned by elements of the form $u_{1} w$ for $u \in V_{+}=\coprod_{n>0} V_{(n)}$ and $w \in W$. Then a conformal full field algebra over $V^{L} \otimes V^{R}$ is equivalent to a module $F$ for the vertex operator algebra $V^{L} \otimes V^{R}$ equipped with an intertwining operator $\mathcal{Y}$ of type $\left(\begin{array}{c}F \\ F F\end{array}\right)$ and an injective module map $\rho: V^{L} \otimes V^{R} \rightarrow F$, satisfying the following conditions:

(1) The identity property: $\mathcal{Y}\left(\rho\left(\mathbf{1}^{L} \otimes \mathbf{1}^{R}\right), x\right)=I_{F}$.

(2) The creation property: For $u \in F, \lim _{x \rightarrow 0} \mathcal{Y}(u, x) \rho\left(\mathbf{1}^{L} \otimes \mathbf{1}^{R}\right)=u$.

(3) The associativity: For $u, v, w \in F$ and $w^{\prime} \in F^{\prime}$,

$$
\begin{aligned}
& \left\langle w^{\prime}, \mathbb{Y}_{\text {an }}^{\mathcal{Y}}\left(u ; z_{1}, \zeta_{1}\right) \mathbb{Y}_{\text {an }}^{\mathcal{Y}}\left(v ; z_{2}, \zeta_{2}\right) w\right\rangle \\
& \quad=\left\langle w^{\prime}, \mathbb{Y}_{\text {an }}^{\mathcal{Y}}\left(\mathbb{Y}_{\text {an }}^{\mathcal{Y}}\left(u ; z_{1}-z_{2}, \zeta_{1}-\zeta_{2}\right) v ; z_{2}, \zeta_{2}\right) w\right\rangle
\end{aligned}
$$

holds when $\left|z_{1}\right|>\left|z_{2}\right|>0$ and $\left|\zeta_{1}\right|>\left|\zeta_{2}\right|>0$.

(4) The single-valuedness property:

$$
e^{2 \pi i\left(L^{L}(0)-L^{R}(0)\right)}=I_{F} .
$$

(5) The skew symmetry:

$$
\mathbb{Y}^{\mathcal{Y}}(u ; 1,1) v=e^{L^{L}(-1)+L^{R}(-1)} \mathbb{Y}^{\mathcal{Y}}\left(v ; e^{\pi i}, e^{-\pi i}\right) u .
$$

Let $V$ be a simple vertex operator algebra satisfying the following:

Condition 1.3 (uniqueness of vacuum). For $n<0, V_{(n)}=0, V_{(0)}=\mathbb{C} 1$ and for an irreducible $V$-module $W$ not isomorphic to $V, W_{(0)}=0$.

Condition 1.4 (complete reducibility). Every $\mathbb{N}$-gradable weak $V$-module is completely reducible.

Condition $1.5\left(C_{2}\right.$-cofiniteness). $V$ is $C_{2}$-cofinite, that is, $\operatorname{dim} V / C_{2}(V)<\infty$, where $C_{2}(V)$ is the subspace of $V$ spanned by elements of the form $u_{-2} v$ for $u, v \in V$.

We now recall the construction of conformal full field algebras over $V \otimes V$ in [HK.

Let $\mathcal{A}$ be the set of equivalence classes of irreducible $V$-modules. For any $a \in \mathcal{A}$, we choose a representative $W^{a}$ from $a$. Then there exists $h_{a} \in \mathbb{R}$ such that $W^{a}=$ $\bigsqcup_{n \in \mathbb{Z}} W_{\left(n+h_{a}\right)}^{a}$.

For a single-valued branch $f_{1}\left(z_{1}, z_{2}\right)$ of a multivalued analytic function in a region $A$, we use $E\left(f_{1}\left(z_{1}, z_{2}\right)\right)$ to denote the multivalued analytic extension together with 
the preferred branch $f_{1}\left(z_{1}, z_{2}\right)$. Let $w_{1}=w_{1}\left(z_{1}, z_{2}\right)$ and $w_{2}=w_{2}\left(z_{1}, z_{2}\right)$ be a change of variables and let $f_{2}\left(z_{1}, z_{2}\right)$ be a branch of $E\left(f_{1}\left(z_{1}, z_{2}\right)\right)$ in a region $B$ containing $w_{1}\left(z_{1}, z_{2}\right)=0$ and $w_{2}\left(z_{1}, z_{2}\right)=0$ such that $A \cap B \neq \emptyset$ and $f_{1}\left(z_{1}, z_{2}\right)=f\left(z_{1}, z_{2}\right)$ for $\left(z_{1}, z_{2}\right) \in A \cap B$. Then we use

$$
\operatorname{Res}_{w_{1}=0 \mid w_{2}} E\left(f_{1}\left(z_{1}, z_{2}\right)\right)
$$

to denote the coefficient of $w_{1}^{-1}$ in the expansion of $f_{2}\left(z_{1}, z_{2}\right)$ as a series in powers of $w_{1}$ whose coefficients are analytic functions of $w_{2}$.

For $a_{1}, a_{2}, a_{3} \in \mathcal{A}, w_{a_{1}} \in W^{a_{1}}, w_{a_{2}} \in W^{a_{2}}, w_{a_{1}^{\prime}} \in\left(W^{a_{1}}\right)^{\prime}, w_{a_{2}}^{\prime} \in\left(W^{a_{1}}\right)^{\prime}$, $\mathcal{Y}_{1} \in \mathcal{V}_{a_{1} a_{2}}^{a_{3}}$ and $\mathcal{Y}_{2} \in \mathcal{V}_{a_{1}^{\prime} a_{2}^{\prime}}^{a_{3}^{\prime}}$, let $\left\langle\mathcal{Y}_{1}, \mathcal{Y}_{2}\right\rangle_{\mathcal{V}_{1} a_{2}}^{a_{3}} \in \mathbb{C}$ be given by

$$
\begin{gathered}
\operatorname{Res}_{1-z_{1}-z_{2}=0 \mid z_{2}}\left(1-z_{1}-z_{2}\right)^{-1} E\left(\left\langlee^{L(1)} \mathcal{Y}_{2}\left(\left(1-z_{1}-z_{2}\right)^{L(0)} \tilde{w}_{a_{1}}^{\prime}, z_{1}\right) \tilde{w}_{a_{2}}^{\prime},\right.\right. \\
\left.\left.e^{L(1)} \mathcal{Y}_{1}\left(\left(1-z_{1}-z_{2}\right)^{L(0)} \tilde{w}_{a_{1}}, z_{2}\right) \tilde{w}_{a_{2}}\right\rangle\right) \\
=\left\langle w_{a_{1}}^{\prime}, w_{a_{1}}\right\rangle\left\langle w_{a_{2}}^{\prime}, w_{a_{2}}\right\rangle\left\langle\mathcal{Y}_{1}, \mathcal{Y}_{2}\right\rangle_{\mathcal{V}_{1} a_{2}}^{a_{3}}
\end{gathered}
$$

It was shown in $\mathrm{HK}$ that $\left\langle\mathcal{Y}_{1}, \mathcal{Y}_{2}\right\rangle_{\mathcal{V}_{a_{1} a_{2}}^{a_{3}}}$ indeed exists. Clearly, $\left\langle\mathcal{Y}_{1}, \mathcal{Y}_{2}\right\rangle_{\mathcal{V}_{a_{1} a_{2}}^{a_{3}}}$ is bilinear in $\mathcal{Y}_{1}$ and $\mathcal{Y}_{2}$. Thus we have a pairing $\langle\cdot, \cdot\rangle_{\mathcal{V}_{a_{1} a_{2}}^{a_{3}}}: \mathcal{V}_{a_{1} a_{2}}^{a_{3}} \otimes \mathcal{V}_{a_{1}^{\prime} a_{2}^{\prime}}^{a^{\prime}} \rightarrow \mathbb{C}$. The following is another main result of [HK]:

Theorem 1.6. The pairing $\langle\cdot, \cdot\rangle_{\mathcal{V}_{a_{1} a_{2}}^{a_{3}}}: \mathcal{V}_{a_{1} a_{2}}^{a_{3}} \otimes \mathcal{V}_{a_{1}^{\prime} a_{2}^{\prime}}^{a_{3}^{\prime}} \rightarrow \mathbb{C}$ is nondegenerate. In particular, $N_{a_{1}^{\prime} a_{2}^{\prime}}^{a^{\prime}}=N_{a_{1} a_{2}}^{a_{3}}$.

Recall that in [H10, an action of $S_{3}$ on the space of intertwining operators for a vertex operator algebra was given. We choose a canonical basis of $\mathcal{V}_{a_{1} a_{2}}^{a_{3}}$ for $a_{1}, a_{2}, a_{3} \in \mathcal{A}$ when one of $a_{1}, a_{2}, a_{3}$ is $e$. For $a \in \mathcal{A}$, we choose $\mathcal{Y}_{e a ; 1}^{a}$ to be the vertex operator $Y_{W^{a}}$ defining the module structure on $W^{a}$ and we choose $\mathcal{Y}_{a e ; 1}^{a}$ to be the intertwining operator defined using the action of $\sigma_{12}$ on $\mathcal{Y}_{e a ; 1}^{a}$, or equivalently, using the skew-symmetry in this case,

$$
\begin{aligned}
\mathcal{Y}_{a e ; 1}^{a}\left(w_{a}, x\right) u & =\sigma_{12}\left(\mathcal{Y}_{e a ; 1}^{a}\right)\left(w_{a}, x\right) u \\
& =e^{x L(-1)} \mathcal{Y}_{e a ; 1}^{a}(u,-x) w_{a} \\
& =e^{x L(-1)} Y_{W^{a}}(u,-x) w_{a}
\end{aligned}
$$

for $u \in V$ and $w_{a} \in W^{a}$. Since $V^{\prime}$ as the contragredient of the irreducible module $V$ is an irreducible $V$-module (see [FHL]) and has a nonzero homogeneous subspace of weight 0 , as a $V$-module it must be isomorphic to $V$. So we have $e^{\prime}=e$. From [FHL, we know that there is a nondegenerate invariant bilinear form $(\cdot, \cdot)$ on $V$ such that $(\mathbf{1}, \mathbf{1})=1$. We choose $\mathcal{Y}_{a a^{\prime} ; 1}^{e}=\mathcal{Y}_{a a^{\prime} ; 1}^{e^{\prime}}$ to be the intertwining operator defined using the action of $\sigma_{23}$ on

$$
\mathcal{Y}_{a a^{\prime} ; 1}^{e^{\prime}}=\sigma_{23}\left(\mathcal{Y}_{a e ; 1}^{a}\right),
$$

that is,

$$
\left(u, \mathcal{Y}_{a a^{\prime} ; 1}^{e}\left(w_{a}, x\right) w_{a^{\prime}}\right)=e^{\pi i h_{a}}\left\langle\mathcal{Y}_{a e ; 1}^{a}\left(e^{x L(1)}\left(e^{-\pi i} x^{-2}\right)^{L(0)} w_{a}, x^{-1}\right) u, w_{a^{\prime}}\right\rangle
$$

for $u \in V, w_{a} \in W^{a}$ and $w_{a^{\prime}} \in W^{a^{\prime}}$. Since the actions of $\sigma_{12}$ and $\sigma_{23}$ generate the 
action of $S_{3}$ on $\mathcal{V}$, we have

$$
\mathcal{Y}_{a^{\prime} a ; 1}^{e}=\sigma_{12}\left(\mathcal{Y}_{a a^{\prime} ; 1}^{e}\right)
$$

for any $a \in \mathcal{A}$.

As in [HK], for $a \in \mathcal{A}$, we let

$$
F_{a}=F\left(\mathcal{Y}_{a e ; 1}^{a} \otimes \mathcal{Y}_{a^{\prime} a ; 1}^{e} ; \mathcal{Y}_{e a ; 1}^{a} \otimes \mathcal{Y}_{a a^{\prime} ; 1}^{e}\right) \neq 0
$$

and we use $\sqrt{F_{a}}$ to denote the square root $\sqrt{\left|F_{a}\right|} e^{\frac{i \arg F_{a}}{2}}$ of $F_{a}$. For $a_{1}, a_{2}, a_{3} \in \mathcal{A}$, consider the modified pairings

$$
\frac{\sqrt{F_{a_{3}}}}{\sqrt{F_{a_{1}}} \sqrt{F_{a_{2}}}}\langle\cdot, \cdot\rangle_{\mathcal{V}_{a_{1} a_{2}}^{a_{3}}} .
$$

These pairings give a nondegenerate bilinear form $(\cdot, \cdot)_{\mathcal{V}}$ on $\mathcal{V}$. For any basis $\left\{\mathcal{Y}_{a_{1} a_{2} ; i}^{a_{3}} \mid i=\ldots, N_{a_{1} a_{2}}^{a_{3}}\right\}$ of $\mathcal{V}_{a_{1} a_{2}}^{a_{3}}$ and any $\sigma \in S_{3},\left\{\sigma\left(\mathcal{Y}_{a_{1} a_{2} ; i}^{a_{3}}\right) \mid i=\ldots, N_{a_{1} a_{2}}^{a_{3}}\right\}$ is a basis of $\sigma\left(\mathcal{V}_{a_{1} a_{2}}^{a_{3}}\right)$.

We have the following result from $\mathrm{HK}$ :

Proposition 1.7. The nondegenerate bilinear form $(\cdot, \cdot)_{\mathcal{V}}$ is invariant with respect to the action of $S_{3}$ on $\mathcal{V}$; that is, for $a_{1}, a_{2}, a_{3} \in \mathcal{A}, \sigma \in S_{3}, \mathcal{Y}_{1} \in \mathcal{V}_{a_{1} a_{2}}^{a_{3}}$ and $\mathcal{Y}_{2} \in \mathcal{V}_{a_{1}^{\prime} a_{2}^{\prime}}^{a_{3}^{\prime}}$,

$$
\left(\sigma\left(\mathcal{Y}_{1}\right), \sigma\left(\mathcal{Y}_{2}\right)\right)_{\mathcal{V}}=\left(\mathcal{Y}_{1}, \mathcal{Y}_{2}\right)_{\mathcal{V}}
$$

Equivalently, for $a_{1}, a_{2}, a_{3} \in \mathcal{A}$

$$
\left\{\frac{\sqrt{F_{a_{1}}} \sqrt{F_{a_{2}}} \sqrt{F_{a_{\sigma-1}(3)}}}{\sqrt{F_{a_{\sigma^{-1}(1)}}} \sqrt{F_{a_{\sigma^{-1}(2)}}} \sqrt{F_{a_{3}}}} \sigma\left(\mathcal{Y}_{a_{1}^{\prime} a_{2}^{\prime} ; a^{\prime}}^{\prime}\right) \mid i=\ldots, N_{a_{1} a_{2}}^{a_{3}}\right\}
$$

is the dual basis of $\left\{\sigma\left(\mathcal{Y}_{a_{1} a_{2} ; i}^{a_{3}}\right) \mid i=\ldots, N_{a_{1} a_{2}}^{a_{3}}\right\}$, where $\left\{\mathcal{Y}_{a_{1} a_{2} ; i}^{a_{3}} \mid i=\ldots, N_{a_{1} a_{2}}^{a_{3}}\right\}$ is a basis of $\mathcal{V}_{a_{1} a_{2}}^{a_{3}}$ and $\left\{\mathcal{Y}_{a_{1}^{\prime} a_{2}^{\prime} ; i}^{\prime \prime a_{3}^{\prime}} \mid i=\ldots, N_{a_{1} a_{2}}^{a_{3}}\right\}$ is its dual basis with respect to the pairing $\langle\cdot, \cdot\rangle_{\mathcal{V}_{1} a_{2}}^{a_{3}}$.

Let

$$
F=\bigoplus_{a \in \mathcal{A}} W^{a} \otimes W^{a^{\prime}}
$$

For $w_{a_{1}} \in W^{a_{1}}, w_{a_{2}} \in W^{a_{2}}, w_{a_{1}^{\prime}} \in W^{a_{1}^{\prime}}$ and $w_{a_{2}^{\prime}} \in W^{a_{2}^{\prime}}$, we define

$$
\begin{aligned}
& \mathbb{Y}\left(\left(w_{a_{1}} \otimes w_{a_{1}^{\prime}}\right) ; z, \bar{z}\right)\left(w_{a_{2}} \otimes w_{a_{2}^{\prime}}\right) \\
& \quad=\sum_{a_{3} \in \mathcal{A}} \sum_{p=1}^{N_{a_{1} a_{2}}^{a_{3}}} \mathcal{Y}_{a_{1} a_{2} ; p}^{a_{3}}\left(w_{a_{1}}, z\right) w_{a_{2}} \otimes \mathcal{Y}_{a_{1}^{\prime} a_{2}^{\prime} ; p}^{\prime a_{3}^{\prime}}\left(w_{a_{1}^{\prime}}, \bar{z}\right) w_{a_{2}^{\prime}} .
\end{aligned}
$$

In [HK], we proved the following result:

Theorem 1.8. The quadruple $(F, \mathbb{Y}, \mathbf{1} \otimes \mathbf{1}, \omega \otimes \mathbf{1}, \mathbf{1} \otimes \omega)$ is a conformal full field algebra over $V \otimes V$. 


\section{Modular INVARIANCE FOR INTERTWINING OPERATOR ALGEBRAS}

In this section, we review the modular invariance of intertwining operator algebras proved in [H7]. We assume in this section that $V$ is a simple vertex operator algebra satisfying Conditions 1.4 and 1.5 and the condition that for $n<0, V_{(n)}=0$ and $V_{(0)}=\mathbb{C} 1$. Note that the last condition is weaker than Condition 1.3. We shall use $Y$ to denote the vertex operator maps for the algebra $V$ and for $V$-modules.

Let $A_{j}, j \in \mathbb{Z}_{+}$, be the complex numbers defined by

$$
\frac{1}{2 \pi i} \log (1+2 \pi i y)=\left(\exp \left(\sum_{j \in \mathbb{Z}_{+}} A_{j} y^{j+1} \frac{\partial}{\partial y}\right)\right) y .
$$

For any $V$-module $W$, we shall denote the operator $\sum_{j \in \mathbb{Z}_{+}} A_{j} L(j)$ on $W$ by $L_{+}(A)$. Then

$$
\exp \left(-\sum_{j \in \mathbb{Z}_{+}} A_{j} L(j)\right)=e^{-L_{+}(A)}
$$

Let

$$
\mathcal{U}(x)=(2 \pi i)^{L(0)} x^{L(0)} e^{-L^{+}(A)} \in(\text { End } W)\{x\},
$$

where $(2 \pi i)^{L(0)}=e^{\left(\log 2 \pi+i \frac{\pi}{2}\right) L(0)}$. Let $B_{j} \in \mathbb{Q}$ for $j \in \mathbb{Z}_{+}$be defined by

$$
\log (1+y)=\left(\exp \left(\sum_{j \in \mathbb{Z}_{+}} B_{j} y^{j+1} \frac{\partial}{\partial y}\right)\right) y .
$$

Then it is easy to see that

$$
\mathcal{U}(x)=x^{L(0)} e^{-L^{+}(B)}(2 \pi i)^{L(0)} .
$$

For any $z \in \mathbb{C}$, we shall denote $e^{2 \pi i z}$ by $q_{z}$ and we shall also use $\mathcal{U}\left(q_{z}\right)$ to denote the map obtained by substituting $e^{2 \pi i z L(0)}$ for $x^{L(0)}$ in $\mathcal{U}(x)$, that is,

$$
\begin{aligned}
\mathcal{U}\left(q_{z}\right) & =(2 \pi i)^{L(0)} e^{2 \pi i z L(0)} e^{-L^{+}(A)} \\
& =e^{2 \pi i z L(0)} e^{-L^{+}(B)}(2 \pi i)^{L(0)}
\end{aligned}
$$

For $V$-modules $W_{i}$ and $\tilde{W}_{i}, i=1, \ldots, n$, intertwining operators $\mathcal{Y}_{i}, i=1, \ldots, n$, of types $\left(\begin{array}{c}\tilde{W}_{i-1} \\ W_{i} \tilde{W}_{i}\end{array}\right)$, respectively, where we use the convention $\tilde{W}_{0}=\tilde{W}_{n}$, and $w_{i} \in W_{i}$, $i=1, \ldots, n$, we shall consider the element

$$
\begin{aligned}
& F_{\mathcal{Y}_{1}, \ldots, \mathcal{Y}_{n}}\left(w_{1}, \ldots, w_{n} ; z_{1}, \ldots, z_{n} ; q\right) \\
& \quad=\operatorname{Tr}_{\tilde{W}_{n}} \mathcal{Y}_{n}\left(\mathcal{U}\left(q_{z_{1}}\right) w_{1}, q_{z_{1}}\right) \cdots \mathcal{Y}_{1}\left(\mathcal{U}\left(q_{z_{n}}\right) w_{n}, q_{z_{n}}\right) q^{L(0)-\frac{c}{24}}
\end{aligned}
$$

of $\mathbb{G}_{\mid q_{1}}|>\cdots>| q_{z_{n}} \mid>0((q))$, where for complex variables $\xi_{1}, \ldots, \xi_{n}, \mathbb{G}_{\left|\xi_{1}\right|>\cdots>\left|\xi_{n}\right|>0}$ is the space of all multivalued analytic functions in $\xi_{1}, \ldots, \xi_{n}$ defined on the region $\left|\xi_{1}\right|>\cdots>\left|\xi_{n}\right|>0$ with preferred branches in the simply connected region $\left|\xi_{1}\right|>\cdots>\left|\xi_{n}\right|>0,0 \leq \arg \xi_{i}<2 \pi, i=1, \ldots, n$.

In [H7], the following result was proved:

Theorem 2.1. In the region $1>\left|q_{z_{1}}\right|>\cdots>\left|q_{z_{n}}\right|>\left|q_{\tau}\right|>0$, the series

$$
F_{\mathcal{Y}_{1}, \ldots, \mathcal{Y}_{n}}\left(w_{1}, \ldots, w_{n} ; z_{1}, \ldots, z_{n} ; q_{\tau}\right)
$$


is absolutely convergent and can be analytically extended to a (multivalued) analytic function in the region given by $\Im(\tau)>0$ (here $\Im(\tau)$ is the imaginary part of $\tau$ ), $z_{i} \neq z_{j}+k \tau+l$ for $i, j=1, \ldots, n, i \neq j, k, l \in \mathbb{Z}$.

We shall denote the (multivalued) analytic extension given in Theorem 2.1 by

$$
\left(\Phi\left(\mathcal{Y}_{1} \otimes \cdots \otimes \mathcal{Y}_{n}\right)\right)\left(w_{1}, \ldots, w_{n} ; z_{1}, \ldots, z_{n} ; q_{\tau}\right) .
$$

Note that in [H], this function is denoted by

$$
\bar{F}_{\mathcal{Y}_{1}, \ldots, \mathcal{Y}_{n}}\left(w_{1}, \ldots, w_{n} ; z_{1}, \ldots, z_{n} ; q_{\tau}\right) .
$$

Here we change the notation to avoid confusion with the notation related to algebraic extensions or to complex conjugations and also for convenience in later sections and for future use.

In [H7], the following genus-one duality results were proved:

Theorem 2.2 (Genus-one commutativity). Let $W_{i}$ and $\tilde{W}_{i}$ be $V$-modules and $\mathcal{Y}_{i}$ intertwining operators of types $\left(\begin{array}{l}\tilde{W}_{i-1} \\ W_{i} \tilde{W}_{i}\end{array}\right)\left(i=1, \ldots, n, \tilde{W}_{0}=\tilde{W}_{n}\right)$, respectively. Then for any $1 \leq k \leq n-1$, there exist $V$-modules $\check{W}_{k}$ and intertwining operators $\check{\mathcal{Y}}_{k}$ and $\check{\mathcal{Y}}_{k+1}$ of types $\left(\begin{array}{c}\check{W}_{k} \\ W_{k} \tilde{W}_{k+1}\end{array}\right)$ and $\left(\begin{array}{c}\tilde{W}_{k-1} \\ W_{k+1} \breve{W}_{k}\end{array}\right)$, respectively, such that

$$
F_{\mathcal{Y}_{1}, \ldots, \mathcal{Y}_{n}}\left(w_{1}, \ldots, w_{n} ; z_{1}, \ldots, z_{n} ; q_{\tau}\right)
$$

and

$$
\begin{array}{r}
F_{\mathcal{Y}_{1}, \ldots, \mathcal{Y}_{k-1}, \check{\mathcal{Y}}_{k+1}, \check{\mathcal{Y}}_{k}, \mathcal{Y}_{k+2} \ldots, \mathcal{Y}_{n}}\left(w_{1}, \ldots, w_{k-1}, w_{k+1}, w_{k}, w_{k+2}, \ldots, w_{n} ;\right. \\
\left.z_{1}, \ldots, z_{k-1}, z_{k+1}, z_{k}, z_{k+2}, \ldots, z_{n} ; q_{\tau}\right)
\end{array}
$$

are analytic extensions of each other, or equivalently,

$$
\begin{aligned}
& \left(\Phi\left(\mathcal{Y}_{1} \otimes \cdots \otimes \mathcal{Y}_{n}\right)\right)\left(w_{1}, \ldots, w_{n} ; z_{1}, \ldots, z_{n} ; \tau\right) \\
& =\left(\Phi\left(\mathcal{Y}_{1} \otimes \cdots \otimes \mathcal{Y}_{k-1} \otimes \check{\mathcal{Y}}_{k+1} \otimes \check{\mathcal{Y}}_{k} \otimes \mathcal{Y}_{k+2} \otimes \cdots \otimes \mathcal{Y}_{n}\right)\right)\left(w_{1}, \ldots, w_{k-1},\right. \\
& \left.\quad w_{k+1}, w_{k}, w_{k+2}, \ldots, w_{n} ; z_{1}, \ldots, z_{k-1}, z_{k+1}, z_{k}, z_{k+2}, \ldots, z_{n} ; \tau\right) .
\end{aligned}
$$

More generally, for any $\sigma \in S_{n}$, there exist $V$-modules $\check{W}_{i}(i=1, \ldots, n)$ and intertwining operators $\check{\mathcal{Y}}_{i}$ of types $\left(\begin{array}{c}\check{W}_{i-1} \\ W_{\sigma(i)} \breve{W}_{i}\end{array}\right)\left(i=1, \ldots, n, \check{W}_{0}=\check{W}_{n}=\tilde{W}_{n}\right)$, respectively, such that

$$
F_{\mathcal{Y}_{1}, \ldots, \mathcal{Y}_{n}}\left(w_{1}, \ldots, w_{n} ; z_{1}, \ldots, z_{n} ; q_{\tau}\right)
$$

and

$$
F_{\check{\mathcal{Y}}_{1}, \ldots, \check{\mathcal{Y}}_{n}}\left(w_{\sigma(1)}, \ldots, w_{\sigma(n)} ; z_{\sigma(1)}, \ldots, z_{\sigma(n)} ; q_{\tau}\right)
$$

are analytic extensions of each other, or equivalently,

$$
\begin{aligned}
& \left(\Phi\left(\mathcal{Y}_{1} \otimes \cdots \otimes \mathcal{Y}_{n}\right)\right)\left(w_{1}, \ldots, w_{n} ; z_{1}, \ldots, z_{n} ; \tau\right) \\
& \quad=\left(\Phi\left(\check{\mathcal{Y}}_{1} \otimes \cdots \otimes \check{\mathcal{Y}}_{n}\right)\right)\left(w_{\sigma(1)}, \ldots, w_{\sigma(n)} ; z_{\sigma(1)}, \ldots, z_{\sigma(n)} ; \tau\right)
\end{aligned}
$$

Theorem 2.3 (Genus-one associativity). Let $W_{i}$ and $\tilde{W}_{i}$ for $i=1, \ldots, n$ be $V$ modules and $\mathcal{Y}_{i}$ intertwining operators of types $\left(\begin{array}{c}\tilde{W}_{i-1} \\ W_{i} \tilde{W}_{i}\end{array}\right)\left(i=1, \ldots, n, \tilde{W}_{0}=\tilde{W}_{n}\right)$, 
respectively. Then for any $1 \leq k \leq n-1$, there exist a $V$-module $\breve{W}_{k}$ and intertwining operators $\check{\mathcal{Y}}_{k}$ and $\check{\mathcal{Y}}_{k+1}$ of types $\left(\begin{array}{c}\check{W}_{k} \\ W_{k} W_{k+1}\end{array}\right)$ and $\left(\begin{array}{c}\tilde{W}_{k-1} \\ \tilde{W}_{k} \tilde{W}_{k+1}\end{array}\right)$, respectively, such that

$$
\begin{gathered}
\left(\Phi\left(\mathcal{Y}_{1} \otimes \cdots \otimes \mathcal{Y}_{k-1} \otimes \check{\mathcal{Y}}_{k+1} \otimes \mathcal{Y}_{k+2} \otimes \cdots \otimes \mathcal{Y}_{n}\right)\right)\left(w_{1}, \ldots, w_{k-1},\right. \\
\left.\check{\mathcal{Y}}\left(w_{k}, z_{k}-z_{k+1}\right) w_{k+1}, w_{k+2}, \ldots, w_{n} ; z_{1}, \ldots, z_{k-1}, z_{k+1}, \ldots, z_{n} ; \tau\right) \\
=\sum_{r \in \mathbb{R}}\left(\Phi\left(\mathcal{Y}_{1} \otimes \cdots \otimes \mathcal{Y}_{k-1} \otimes \check{\mathcal{Y}}_{k+1} \otimes \mathcal{Y}_{k+2} \otimes \cdots \otimes \mathcal{Y}_{n}\right)\right)\left(w_{1}, \ldots, w_{k-1},\right. \\
\left.\quad P_{r}\left(\check{\mathcal{Y}}\left(w_{k}, z_{k}-z_{k+1}\right) w_{k+1}\right), w_{k+2}, \ldots, w_{n} ; z_{1}, \ldots, z_{k-1}, z_{k+1}, \ldots, z_{n} ; \tau\right)
\end{gathered}
$$

is absolutely convergent when $1>\left|q_{z_{1}}\right|>\cdots>\left|q_{z_{k-1}}\right|>\left|q_{z_{k+1}}\right|>\cdots>\left|q_{z_{n}}\right|>$ $\left|q_{\tau}\right|>0$ and $1>\left|q_{\left(z_{k}-z_{k+1}\right)}-1\right|>0$ and is convergent to

$$
\left(\Phi\left(\mathcal{Y}_{1} \otimes \cdots \otimes \mathcal{Y}_{n}\right)\right)\left(w_{1}, \ldots, w_{n} ; z_{1}, \ldots, z_{n} ; \tau\right)
$$

when $1>\left|q_{z_{1}}\right|>\cdots>\left|q_{z_{n}}\right|>\left|q_{\tau}\right|>0$ and $\left|q_{\left(z_{k}-z_{k+1}\right)}\right|>1>\left|q_{\left(z_{k}-z_{k+1}\right)}-1\right|>0$.

Let $W_{i}$ be $V$-modules and $w_{i} \in W_{i}$ for $i=1, \ldots, n$. For any $V$-modules $\tilde{W}_{i}$ and any intertwining operators $\mathcal{Y}_{i}, i=1, \ldots, n$, of types $\left(\begin{array}{c}\tilde{W}_{i-1} \\ W_{i} \tilde{W}_{i}\end{array}\right)$, respectively, we have a genus-one correlation function

$$
\left(\Phi\left(\mathcal{Y}_{1} \otimes \cdots \otimes \mathcal{Y}_{n}\right)\right)\left(w_{1}, \ldots, w_{n} ; z_{1}, \ldots, z_{n} ; \tau\right) .
$$

Note that these multivalued functions actually have preferred branches in the region $1>\left|q_{z_{1}}\right|>\cdots>\left|q_{z_{n}}\right|>\left|q_{\tau}\right|>0$ given by the intertwining operators $\mathcal{Y}_{1}, \ldots, \mathcal{Y}_{n}$. Thus linear combinations of these functions make sense. For fixed $V$-modules $W_{i}$ and $w_{i} \in W_{i}$ for $i=1, \ldots, n$, denote the vector space spanned by all such functions by $\mathcal{F}_{w_{1}, \ldots, w_{n}}$. For any single-valued analytic function $f(\tau)$ of $\tau$ and any $r \in \mathbb{R}$, we choose a branch of the multivalued analytic function $f(\tau)^{r}$ to be $e^{r \log f(\tau)}$. The following theorem is one of the main results of [H7]:

Theorem 2.4. For any $V$-modules $\tilde{W}_{i}$ and any intertwining operators $\mathcal{Y}_{i}(i=$ $1, \ldots, n)$ of types $\left(\begin{array}{c}\tilde{W}_{i-1} \\ W_{i} \tilde{W}_{i}\end{array}\right)$, respectively, and any

$$
\begin{gathered}
\left(\begin{array}{cc}
\alpha & \beta \\
\gamma & \delta
\end{array}\right) \in S L(2, \mathbb{Z}), \\
\left(\Phi\left(\mathcal{Y}_{1} \otimes \cdots \otimes \mathcal{Y}_{n}\right)\right)\left(\left(\frac{1}{\gamma \tau+\delta}\right)^{L(0)} w_{1}, \ldots,\left(\frac{1}{\gamma \tau+\delta}\right)^{L(0)} w_{n} ;\right. \\
\left.\frac{z_{1}}{\gamma \tau+\delta}, \ldots, \frac{z_{n}}{\gamma \tau+\delta} ; \frac{\alpha \tau+\beta}{\gamma \tau+\delta}\right)
\end{gathered}
$$

is in $\mathcal{F}_{w_{1}, \ldots, w_{n}}$.

\section{Genus-one Correlation functions and modular invariance For CONFORMAL FULL FIELD ALGEBRAS}

Let $V^{L}$ and $V^{R}$ be simple vertex operator algebras satisfying Conditions 1.3, 1.4 and 1.5. Let $F$ be a conformal full field algebra over $V^{L} \otimes V^{R}$. In particular, $F$ is $\mathbb{R} \times \mathbb{R}$-graded; that is, $F=\coprod_{r, s \in \mathbb{R}} F_{(r, s)}$, where $F_{(r, s)}$ for $r, s \in \mathbb{R}$ are eigenspaces 
for the operators $L^{L}(0)$ and $L^{R}(0)$ with eigenvalues $r$ and $s$, respectively. For a linear map $f: F \rightarrow \bar{F}$, we define the $q$ - $\bar{q}$-trace of $f$ to be

$$
\operatorname{Tr}_{F} f q^{L^{L}(0)-\frac{c^{L}}{24}} \bar{q}^{L^{R}(0)-\frac{c^{R}}{24}}=\sum_{r, s \in \mathbb{R}} \operatorname{Tr}_{F_{(m, n)}} f q^{r-\frac{c^{L}}{24}} \bar{q}^{s-\frac{c^{R}}{24}}
$$

As in [HK], we choose the branches of the functions $z^{r}$ and $\bar{z}^{s}$ for $r, s \in \mathbb{R}$ to be $e^{r \log z}$ and $e^{s \overline{\log z}}$, respectively. On the other hand, for the functions $\left(e^{2 \pi i z}\right)^{r}$ and $\left(\overline{e^{2 \pi i z}}\right)^{s}=\left(e^{-2 \pi i \bar{z}}\right)^{s}$ for $r, s \in \mathbb{R}$, we choose their branches to be $e^{2 \pi i r z}$ and $e^{-2 \pi i s \bar{z}}$, respectively. For multivalued analytic functions obtained from these functions using products and sums, we choose their branches to be the ones obtained from the branches we choose above using the same operations.

Now let

$$
\begin{aligned}
\mathcal{U}^{L}\left(e^{2 \pi i z}\right) & =(2 \pi i)^{L^{L}(0)}\left(e^{2 \pi i z}\right)^{L^{L}(0)} e^{-L_{+}^{L}(A)} \\
& =(2 \pi i)^{L^{L}(0)} e^{2 \pi i z L^{L}(0)} e^{-L_{+}^{L}(A)}, \\
\mathcal{U}^{R}\left(e^{2 \pi i z}\right) & =(2 \pi i)^{L^{R}(0)}\left(e^{2 \pi i z}\right)^{L^{R}(0)} e^{-L_{+}^{R}(A)} \\
& =(2 \pi i)^{L^{R}(0)} e^{2 \pi i z L^{R}(0)} e^{-L_{+}^{R}(A)},
\end{aligned}
$$

where $L_{+}^{L}(A)=\sum_{j \in \mathbb{Z}_{+}} A_{j} L^{L}(j)$ and $L_{+}^{R}(A)=\sum_{j \in \mathbb{Z}_{+}} A_{j} L^{R}(j)$. By (2.1) and our choice of the branches of the functions $\left(e^{2 \pi i z}\right)^{r}$ and $\left(\overline{e^{2 \pi i z}}\right)^{s}=\left(e^{-2 \pi i \bar{z}}\right)^{s}$ above, we have

$$
\begin{aligned}
& \overline{\mathcal{U}^{R}\left(e^{2 \pi i z}\right)}=\overline{\left(e^{2 \pi i z}\right)^{L^{R}(0)} e^{-L_{+}^{L}(B)}(2 \pi i)^{L^{R}(0)}} \\
& =\overline{\left(e^{2 \pi i z}\right)^{L^{R}(0)} e^{-L_{+}^{L}(B)}(2 \pi)^{L^{R}(0)} e^{\frac{\pi i}{2} L^{R}(0)}} \\
& \left.=\overline{\left(e^{-2 \pi i z}\right.}\right)^{L^{R}(0)} e^{-L_{+}^{L}(B)}(2 \pi)^{L^{R}(0)} e^{-\frac{\pi i}{2} L^{R}(0)} \\
& =\left(e^{-2 \pi i \bar{z}}\right)^{L^{R}(0)} e^{-L_{+}^{L}(B)}(2 \pi)^{L^{R}(0)} e^{\frac{\pi i}{2} L^{R}(0)} e^{-\pi i L^{R}(0)} \\
& =\mathcal{U}^{R}\left(e^{-2 \pi i \bar{z}}\right) e^{-\pi i L^{R}(0)} \text {. }
\end{aligned}
$$

For any $u \in F$ and $z \in \mathbb{C}^{\times}$, we shall call the operator

$$
\mathbb{Y}\left(\mathcal{U}^{L}\left(e^{2 \pi i z}\right) \overline{\mathcal{U}^{R}\left(e^{2 \pi i z}\right)} u ; e^{2 \pi i z_{1}}, \overline{e^{2 \pi i z}}\right): F \rightarrow \bar{F}
$$

a geometrically-modified full vertex operator. For $u_{1}, \ldots, u_{k} \in F$ and $z_{1}, \ldots, z_{k} \in \mathbb{C}$ satisfying $\left|e^{2 \pi i z_{1}}\right|>\cdots>\left|e^{2 \pi i z_{k}}\right|>0$, the product

$$
\begin{aligned}
\mathbb{Y}\left(\mathcal{U}^{L}\left(e^{2 \pi i z_{1}}\right) \overline{\mathcal{U}^{R}\left(e^{2 \pi i z_{1}}\right)} u_{1} ; e^{2 \pi i z_{1}}, \overline{e^{2 \pi i z_{1}}}\right) \\
\cdots \mathbb{Y}\left(\mathcal{U}^{L}\left(e^{2 \pi i z_{k}}\right) \overline{\mathcal{U}^{R}\left(e^{2 \pi i z_{k}}\right)} u_{1} ; e^{2 \pi i z_{k}}, \overline{e^{2 \pi i z_{k}}}\right) \\
=\mathbb{Y}\left(\mathcal{U}^{L}\left(e^{2 \pi i z_{1}}\right) \mathcal{U}^{R}\left(e^{-2 \pi i \bar{z}_{1}}\right) e^{-\pi i L^{R}(0)} u_{1} ; e^{2 \pi i z_{1}}, e^{-2 \pi i \bar{z}_{1}}\right) \cdot \\
\cdots \mathbb{Y}\left(\mathcal{U}^{L}\left(e^{2 \pi i z_{k}}\right) \mathcal{U}^{R}\left(e^{-2 \pi i \bar{z}_{k}}\right) e^{-\pi i L^{R}(0)} u_{1} ; e^{2 \pi i z_{k}}, e^{-2 \pi i \bar{z}_{k}}\right)
\end{aligned}
$$


of geometrically-modified full vertex operators is a linear map from $F$ to $\bar{F}$. So we have the $q-\bar{q}$-trace

$$
\begin{gathered}
\operatorname{Tr}_{F} \mathbb{Y}\left(\mathcal{U}^{L}\left(e^{2 \pi i z_{1}}\right) \overline{\mathcal{U}^{R}\left(e^{2 \pi i z_{1}}\right)} u_{1} ; e^{2 \pi i z_{1}}, \overline{e^{2 \pi i z_{1}}}\right) \cdot \\
\ldots \mathbb{Y}\left(\mathcal{U}^{L}\left(e^{2 \pi i z_{1}}\right) \overline{\mathcal{U}^{R}\left(e^{2 \pi i z_{k}}\right)} u_{1} ; e^{2 \pi i z_{1}}, \overline{e^{2 \pi i z_{k}}}\right) q^{L^{L}(0)-\frac{c^{L}}{24}} \bar{q}^{L^{R}(0)-\frac{c^{R}}{24}} \\
=\operatorname{Tr}_{F} \mathbb{Y}\left(\mathcal{U}^{L}\left(e^{2 \pi i z_{1}}\right) \mathcal{U}^{R}\left(e^{-2 \pi i \bar{z}_{1}}\right) e^{-\pi i L^{R}(0)} u_{1} ; e^{2 \pi i z_{1}}, e^{-2 \pi i \bar{z}_{1}}\right) \cdot \\
\cdots \mathbb{Y}\left(\mathcal{U}^{L}\left(e^{2 \pi i z_{1}}\right) \mathcal{U}^{R}\left(e^{-2 \pi i \bar{z}_{k}}\right) e^{-\pi i L^{R}(0)} u_{1} ; e^{2 \pi i z_{1}}, e^{-2 \pi i \bar{z}_{k}}\right) \cdot \\
\cdot q^{L^{L}(0)-\frac{c^{L}}{24}} \bar{q}^{L^{R}(0)-\frac{c^{R}}{24}} .
\end{gathered}
$$

As a module for the vertex operator algebra $V^{L} \otimes V^{R}, F$ is a direct sum of irreducible modules for $V^{L} \otimes V^{R}$. Let $\mathcal{A}^{L}\left(\mathcal{A}^{R}\right)$ be the set of equivalence classes of irreducible modules for $V^{L}\left(V^{R}\right)$. For each $a^{L} \in \mathcal{A}^{L}\left(a^{R} \in \mathcal{A}^{R}\right)$, choose a representative $W^{a^{L}}\left(W^{a^{R}}\right)$ of $a^{L}\left(a^{R}\right)$. Then there exist a positive integer $N$ and maps $r^{L}:\{1, \ldots, N\} \rightarrow \mathcal{A}^{L}, r^{R}:\{1, \ldots, N\} \rightarrow \mathcal{A}^{R}$ such that $F$ is isomorphic as a $V^{L} \otimes V^{R}$-module to $\coprod_{n=1}^{N} W^{r^{L}(n)} \otimes W^{r^{R}(n)}$. We now shall identify the vector space $F$ with this $V^{L} \otimes V^{R}$-module. Then the full vertex operator map can be written as

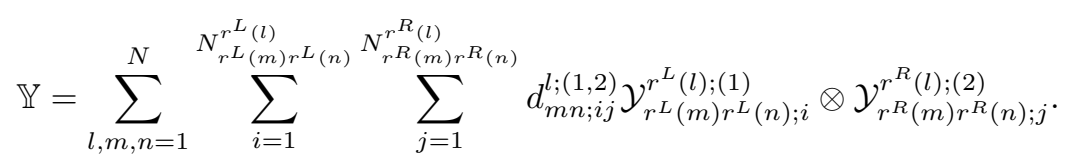

where $d_{m n ; i j}^{l ;(1,2)} \in \mathbb{C}$ for $l, m, n=1, \ldots, N, i=1, \ldots, N_{r^{L}(m) r^{L}(n)}^{r^{L}(l)}, j=1, \ldots$, $N_{r^{R}(m) r^{R}(n)}^{r^{R}(l)}$, and $p, q$ any indices, and

$$
\left\{\mathcal{Y}_{r^{L}(m) r^{L}(n) ; i}^{r^{L}(l) ;(1)} \mid i=1, \ldots, N_{r^{L}(m) r^{L}(n)}^{r^{L}(l)}\right\}
$$

and

$$
\left\{\mathcal{Y}_{r^{R}(m) r^{R}(n) ; j}^{r^{R}(l) ;(2)} \mid i=1, \ldots, N_{r^{R}(m) r^{R}(n)}^{r^{R}(l)}\right\}
$$

for $l, m, n=1, \ldots, N$ are bases of $\mathcal{V}_{r^{L}(m) r^{L}(n)}^{r^{L}(l)}$ and $\mathcal{V}_{r^{R}(m) r^{R}(n)}^{r^{R}(l)}$, respectively.

For $\tau \in \mathbb{H}$, let $q_{\tau}=e^{2 \pi i \tau}$. We have:

Proposition 3.1. For $u_{1}, \ldots, u_{k} \in F$, the $q-\bar{q}$-trace (3.1) with $q=q_{\tau}$ is absolutely convergent when $1>\left|e^{2 \pi i z_{1}}\right|>\cdots>\left|e^{2 \pi i z_{k}}\right|>\left|q_{\tau}\right|>0$. Moreover, the sum of this $q-\bar{q}$-trace can be analytically extended to a multivalued analytic function of $z_{1}, \xi_{1}, \ldots, z_{k}, \xi_{k}, \tau$ and $\sigma$ in the region given by $z_{i} \neq z_{j}+m+n \tau$ for $i \neq j$ and $m, n \in \mathbb{Z}$ and $\xi_{i} \neq \xi_{j}+m+n \sigma$ for $i \neq j$ and $m, n \in \mathbb{Z}$, with the sum of the $q-\bar{q}$-trace (3.1) with $q=q_{\tau}$ as a preferred value at the special points

$$
\left(z_{1}, \xi_{1}=\bar{z}_{1}, \ldots, z_{k}, \xi_{k}=\bar{z}_{k} ; \tau, \sigma=-\bar{\tau}\right)
$$

satisfying $1>\left|e^{2 \pi i z_{1}}\right|>\cdots>\left|e^{2 \pi i z_{k}}\right|>\left|q_{\tau}\right|>0$.

Proof. This result follows immediately from (3.2) and the convergence and analytic extension properties for $q$ - or $\bar{q}$-traces of intertwining operators for the vertex operator algebras $V^{L}$ and $V^{R}$. 
Recall from [HK] the (genus-zero) correlation functions

$$
m_{k}\left(u_{1}, \ldots, u_{k} ; z_{1}, \bar{z}_{1}, \ldots, z_{k}, \bar{z}_{k}\right)
$$

for $k \in \mathbb{Z}_{+}$and $u_{1}, \ldots, u_{k} \in F$ and their analytic extensions

$$
E(m)_{k}\left(u_{1}, \ldots, u_{k} ; z_{1}, \zeta_{1}, \ldots, z_{k}, \zeta_{k}\right)
$$

The functions

$$
\begin{aligned}
& m_{k}\left(\mathcal{U}^{L}\left(e^{2 \pi i z_{1}}\right) \mathcal{U}^{R}\left(e^{-2 \pi i \bar{z}_{1}}\right) e^{-\pi i L^{R}(0)} u_{1},\right. \\
& \left.3.3) \quad \ldots, \mathcal{U}^{L}\left(e^{2 \pi i z_{k}}\right) \mathcal{U}^{R}\left(e^{-2 \pi i \bar{z}_{k}}\right) e^{-\pi i L^{R}(0)} u_{k} ; e^{2 \pi i z_{1}}, e^{-2 \pi i \bar{z}_{1}}, \ldots, e^{2 \pi i z_{k}}, e^{-2 \pi i \bar{z}_{k}}\right)
\end{aligned}
$$

are called geometrically-modified (genus-zero) correlation functions.

Corollary 3.2. For $u_{1}, \ldots, u_{k} \in F$,

$$
\begin{gathered}
\operatorname{Tr}_{F} E(m)_{k}\left(\mathcal{U}^{L}\left(e^{2 \pi i z_{1}}\right) \mathcal{U}^{R}\left(e^{-2 \pi i \xi_{1}}\right) e^{-\pi i L^{R}(0)} u_{1},\right. \\
\ldots, \mathcal{U}^{L}\left(e^{2 \pi i z_{k}}\right) \mathcal{U}^{R}\left(e^{-2 \pi i \xi_{k}}\right) e^{-\pi i L^{R}(0)} u_{k} ; e^{2 \pi i z_{1}}, e^{2 \pi i \xi_{1}}, \\
\left.\ldots, e^{2 \pi i z_{k}}, e^{2 \pi i \xi_{k}}\right) q_{\tau}^{L^{L}(0)-\frac{c^{L}}{24}} q_{\sigma}^{L^{R}(0)-\frac{c^{R}}{24}}
\end{gathered}
$$

is absolutely convergent to a multivalued analytic function of $z_{1}, \xi_{1}, \ldots, z_{k}, \xi_{k}, \tau$ and $\sigma$ in the region given by $1>\left|e^{2 \pi i z_{1}}\right|, \ldots,\left|e^{2 \pi i z_{k}}\right|>\left|q_{\tau}\right|>0,1>\left|e^{2 \pi i \xi_{1}}\right|, \ldots$, $\left|e^{2 \pi i \xi_{k}}\right|>\left|q_{\sigma}\right|>0, z_{i} \neq z_{j}$ and $\xi_{i} \neq \xi_{j}$ for $i \neq j$, and can be analytically extended to a multivalued analytic function of $z_{1}, \xi_{1}, \ldots, z_{k}, \xi_{k}, \tau$ and $\sigma$ in the region given by $z_{i} \neq z_{j}+m+n \tau$ for $i \neq j$ and $m, n \in \mathbb{Z}$ and $\xi_{i} \neq \xi_{j}+m+n \sigma$ for $i \neq j$ and $m, n \in \mathbb{Z}$. In particular, the $q_{\tau}-q_{\bar{\tau}}$-trace

$$
\begin{gathered}
\operatorname{Tr}_{F} m_{k}\left(\mathcal{U}^{L}\left(e^{2 \pi i z_{1}}\right) \mathcal{U}^{R}\left(e^{-2 \pi i \bar{z}_{1}}\right) e^{-\pi i L^{R}(0)} u_{1},\right. \\
\ldots, \mathcal{U}^{L}\left(e^{2 \pi i z_{k}}\right) \mathcal{U}^{R}\left(e^{-2 \pi i \bar{z}_{k}}\right) e^{-\pi i L^{R}(0)} u_{k} ; e^{2 \pi i z_{1}}, e^{-2 \pi i \bar{z}_{1}}, \\
\left.\ldots, e^{2 \pi i z_{k}}, e^{-2 \pi i \bar{z}_{k}}\right) q_{\tau}^{L^{L}(0)-\frac{c^{L}}{24}} \bar{q}_{\tau}^{L^{R}(0)-\frac{c^{R}}{24}}
\end{gathered}
$$

is absolutely convergent when $1>\left|e^{2 \pi i z_{1}}\right|, \ldots,\left|e^{2 \pi i z_{k}}\right|>\left|q_{\tau}\right|>0$ and $z_{i} \neq z_{j}$ for $i \neq j$.

Proof. The terms in the series (3.4) are the analytic extensions of the terms in (3.1). From Proposition 3.1, (3.1) is absolutely convergent in the region given by $1>\left|e^{2 \pi i z_{1}}\right|, \ldots,\left|e^{2 \pi i z_{k}}\right|>\left|q_{\tau}\right|>0$ and can be analytically extended to the region given by $z_{i} \neq z_{j}+m+n \tau$ for $i \neq j$ and $m, n \in \mathbb{Z}$ and $\xi_{i} \neq \xi_{j}+m+n \sigma$ for $i \neq j$ and $m, n \in \mathbb{Z}$. We also know that the resulting analytic extension can be expanded as a series in powers of $q_{\tau}$ and $q_{\sigma}$ in the region given by $1>\left|e^{2 \pi i z_{1}}\right|, \ldots,\left|e^{2 \pi i z_{k}}\right|>$ $\left|q_{\tau}\right|>0,1>\left|e^{2 \pi i \xi_{1}}\right|, \ldots,\left|e^{2 \pi i \xi_{k}}\right|>\left|q_{\sigma}\right|>0, z_{i} \neq z_{j}$ for $i \neq j$ and $\xi_{i} \neq \xi_{j}$ for $i \neq j$ and $m, n \in \mathbb{Z}$. Thus the terms of this expansion must be equal to the terms in the series (3.4). In particular, (3.4) is absolutely convergent.

We shall denote the sum of the series (3.5)), in the region given by $1>\left|e^{2 \pi i z_{1}}\right|, \ldots$, $\left|e^{2 \pi i z_{k}}\right|>\left|q_{\tau}\right|>0$ and $z_{i} \neq z_{j}$ for $i \neq j$, by

$$
m_{k}^{(1)}\left(u_{1}, \ldots, u_{k} ; z_{1}, \bar{z}_{1}, \ldots, z_{k}, \bar{z}_{k} ; \tau, \bar{\tau}\right),
$$

where we use the superscript (1) to indicate that this corresponds to a genus 1 surface. The limits of this function of $\left(z_{1}, \ldots, z_{k} ; \tau\right)$ exist when $\left(z_{1}, \ldots, z_{k} ; \tau\right)$ 
approaches points satisfying $\left|e^{2 \pi i z_{l}}\right|=1$ or $\left|e^{2 \pi i z_{l}}\right|=\left|q_{\tau}\right|$ for some $l$ and $z_{i} \neq$ $z_{j}+m+n \tau$ for $i \neq j$ and $m, n \in \mathbb{Z}$, since (3.6) can be analytically extended to a multivalued analytic function of $z_{1}, \xi_{1}, \ldots, z_{k}, \xi_{k}, \tau$ and $\sigma$ in the region given by $z_{i} \neq z_{j}+m+n \tau$ for $i \neq j$ and $m, n \in \mathbb{Z}$ and $\xi_{i} \neq \xi_{j}+m+n \sigma$ for $i \neq j$ and $m, n \in \mathbb{Z}$. So we can extend the function (3.6) to a function defined also on limit points. We use the same notation to denote the extension. We have:

Proposition 3.3. For any $m, n \in \mathbb{Z}$ and $i=1, \ldots, k$,

$$
\begin{aligned}
& m_{k}^{(1)} \begin{array}{l}
\left(u_{1}, \ldots, u_{k} ; z_{1}, \bar{z}_{1}, \ldots, z_{i-1}, \bar{z}_{i-1}, z_{i}+m+n \tau, \bar{z}_{i}+m+n \bar{\tau}\right. \\
\left.z_{i+1}, \bar{z}_{i+1}, \ldots, z_{k}, \bar{z}_{k} ; \tau, \bar{\tau}\right) \\
3.7)=m_{k}^{(1)}\left(u_{1}, \ldots, u_{k} ; z_{1}, \bar{z}_{1}, \ldots, z_{k}, \bar{z}_{k} ; \tau, \bar{\tau}\right)
\end{array}
\end{aligned}
$$

when both sides are defined.

Proof. Note that (3.3) is a single-valued function of $e^{2 \pi i z_{1}}, \ldots, e^{2 \pi i z_{k}}$. Thus we see that (3.7) holds when $n=0$.

Now from the permutation property for conformal full field algebras over $V^{L} \otimes$ $V^{R}, \mathcal{U}^{L}\left(e^{2 \pi i z_{i}}\right) \mathcal{U}^{R}\left(e^{-2 \pi i \bar{z}_{i}}\right) e^{-\pi i L^{R}(0)} u_{i}$ and $e^{2 \pi i z_{i}}, e^{-2 \pi i \bar{z}_{i}}$ in (3.3) can be moved to the right of $\mathcal{U}^{L}\left(e^{2 \pi i z_{k}}\right) \mathcal{U}^{R}\left(e^{-2 \pi i \bar{z}_{k}}\right) e^{-\pi i L^{R}(0)} u_{k}$ and $e^{2 \pi i z_{k}}, e^{-2 \pi i \bar{z}_{k}}$, respectively. So we can assume that $i=k$. In this case, when $1>\left|e^{2 \pi i z_{1}}\right|>\cdots>\left|e^{2 \pi i z_{k}}\right|>\left|q_{\tau}\right|>0$, we have

$$
\begin{aligned}
& m_{k}^{(1)}\left(u_{1}, \ldots, u_{k} ; z_{1}, \bar{z}_{1}, \ldots, z_{k}, \bar{z}_{k} ; \tau, \bar{\tau}\right) \\
& =\operatorname{Tr}_{F} \mathbb{Y}\left(\mathcal{U}^{L}\left(e^{2 \pi i z_{1}}\right) \overline{\mathcal{U}^{R}\left(e^{2 \pi i z_{1}}\right)} u_{1} ; e^{2 \pi i z_{1}}, \overline{e^{2 \pi i z_{1}}}\right) . \\
& \cdots \mathbb{Y}\left(\mathcal{U}^{L}\left(e^{2 \pi i z_{k}}\right) \overline{\mathcal{U}^{R}\left(e^{2 \pi i z_{k}}\right)} u_{k} ; e^{2 \pi i z_{k}}, \overline{e^{2 \pi i z_{k}}}\right) q_{\tau}^{L^{L}(0)-\frac{c^{L}}{24}} \bar{q}_{\tau}^{L^{R}(0)-\frac{c^{R}}{24}} \\
& =\operatorname{Tr}_{F} \mathbb{Y}\left(\mathcal{U}^{L}\left(e^{2 \pi i z_{1}}\right) \overline{\mathcal{U}^{R}\left(e^{2 \pi i z_{1}}\right)} u_{1} ; e^{2 \pi i z_{1}}, \overline{e^{2 \pi i z_{1}}}\right) \cdots q_{\tau}^{L^{L}(0)-\frac{c^{L}}{24}} \bar{q}_{\tau}^{L^{R}(0)-\frac{c^{R}}{24}} . \\
& \text {. } \mathbb{Y}\left(\mathcal{U}^{L}\left(e^{2 \pi i\left(z_{k}-\tau\right)}\right) \overline{\mathcal{U}^{R}\left(e^{2 \pi i\left(z_{k}-\tau\right)}\right)} u_{k} ; e^{2 \pi i\left(z_{k}-\tau\right)}, \overline{e^{2 \pi i\left(z_{k}-\tau\right)}}\right) \\
& =\operatorname{Tr}_{F} \mathbb{Y}\left(\mathcal{U}^{L}\left(e^{2 \pi i\left(z_{k}-\tau\right)}\right) \overline{\mathcal{U}^{R}\left(e^{2 \pi i\left(z_{k}-\tau\right)}\right)} u_{k} ; e^{2 \pi i\left(z_{k}-\tau\right)}, \overline{e^{2 \pi i\left(z_{k}-\tau\right)}}\right) . \\
& \cdot \mathbb{Y}\left(\mathcal{U}^{L}\left(e^{2 \pi i z_{1}}\right) \overline{\mathcal{U}^{R}\left(e^{2 \pi i z_{1}}\right)} u_{1} ; e^{2 \pi i z_{1}}, \overline{e^{2 \pi i z_{1}}}\right) \cdot \\
& \cdots \mathbb{Y}\left(\mathcal{U}^{L}\left(e^{2 \pi i z_{k-1}}\right) \overline{\mathcal{U}^{R}\left(e^{2 \pi i z_{k-1}}\right)} u_{k-11} ; e^{2 \pi i z_{k-1}}, \overline{e^{2 \pi i z_{k-1}}}\right) . \\
& \cdot q_{\tau}^{L^{L}(0)-\frac{c^{L}}{24}} \bar{q}_{\tau}^{L^{R}(0)-\frac{c}{24}} \text {. }
\end{aligned}
$$

When $1>\left|e^{2 \pi i\left(z_{k}-\tau\right)}\right|>\left|e^{2 \pi i z_{1}}\right|>\cdots>\left|e^{2 \pi i z_{k-1}}\right|>\left|q_{\tau}\right|>0$, the right-hand side of (3.8) is actually equal to

$$
\begin{gathered}
m_{k}^{(1)}\left(u_{k}, u_{1}, \ldots, u_{k-1} ; z_{k}-\tau, \bar{z}_{k}-\bar{\tau}, z_{1}, \bar{z}_{1}, \ldots, z_{k-1}, \bar{z}_{k-1} ; \tau, \bar{\tau}\right) \\
=m_{k}^{(1)}\left(u_{1}, \ldots, u_{k} ; z_{1}, \bar{z}_{1}, \ldots, z_{k}-\tau, \bar{z}_{k}-\bar{\tau} ; \tau, \bar{\tau}\right) .
\end{gathered}
$$

Since the left-hand side of (3.8) and the right-hand side of (3.9) are determined uniquely by their values in the regions $1>\left|e^{2 \pi i z_{1}}\right|>\cdots>\left|e^{2 \pi i z_{k}}\right|>\left|q_{\tau}\right|>0$ and $1>\left|e^{2 \pi i\left(z_{k}-\tau\right)}\right|>\left|e^{2 \pi i z_{1}}\right|>\cdots>\left|e^{2 \pi i z_{k-1}}\right|>\left|q_{\tau}\right|>0$, respectively, we see that the left-hand side of (3.8) must be equal to the right-hand side of (3.9), proving (3.7) in the case of $m=0$.

Combining the cases $m=0$ and $n=0$, the proposition is proved. 
From this result and the $L^{L}(-1)$ - and $L^{R}(-1)$-derivative properties, we immediately obtain the following:

Corollary 3.4. For $u_{1}, \ldots, u_{k} \in F$, there exists a unique smooth function, still denoted by (3.6), of $z_{1}, \ldots, z_{k}$ and $\tau$ in the region $z_{i}-z_{j} \neq m+n \tau$ for $i \neq j$ and $\Im(\tau)>0$ such that (3.7) holds and in the region given by $1>\left|e^{2 \pi i z_{1}}\right|, \ldots,\left|e^{2 \pi i z_{k}}\right|>$ $\left|q_{\tau}\right|>0$ and $z_{i} \neq z_{j}$ for $i \neq j$, this function is equal to (3.6).

Now we discuss the modular invariance of these functions. For any single-valued analytic function $f(\tau)$ of $\tau$ and any $r \in R$, we choose branches of the multivalued analytic functions $f(\tau)^{r}$ and $(\overline{f(\tau)})^{r}$ to be $e^{r \log f(\tau)}$ and $e^{r \overline{\log f(\tau)}}$ and still denote them by $f(\tau)^{r}$ and $(\overline{f(\tau)})^{r}$. In particular, for $\alpha, \beta, \gamma, \delta \in \mathbb{R}$,

$$
\left(\frac{\alpha \tau+\beta}{\gamma \tau+\delta}\right)^{r}=e^{r \log \left(\frac{\alpha \tau+\beta}{\gamma \tau+\delta}\right)}
$$

and

$$
\begin{aligned}
\left(\frac{\alpha \bar{\tau}+\beta}{\gamma \bar{\tau}+\delta}\right)^{r} & =\left(\overline{\frac{\alpha \tau+\beta}{\gamma \tau+\delta}}\right)^{r} \\
& =e^{r \overline{\log \left(\frac{\alpha \tau+\beta}{\gamma \tau+\delta}\right)}}
\end{aligned}
$$

Definition 3.5. For $u_{1}, \ldots, u_{k} \in V$, the function (3.6) is invariant under the action of

$$
\left(\begin{array}{ll}
\alpha & \beta \\
\gamma & \delta
\end{array}\right) \in S L(2, \mathbb{Z})
$$

if

$$
\begin{gathered}
m_{k}^{(1)}\left(\left(\frac{1}{\gamma \tau+\delta}\right)^{L^{L}(0)}\left(\frac{1}{\gamma \bar{\tau}+\delta}\right)^{L^{R}(0)} u_{1}, \ldots,\left(\frac{1}{\gamma \tau+\delta}\right)^{L^{L}(0)}\left(\frac{1}{\gamma \bar{\tau}+\delta}\right)^{L^{R}(0)} u_{k}\right. \\
\left.\frac{z_{1}}{\gamma \tau+\delta}, \frac{\bar{z}_{1}}{\gamma \bar{\tau}+\delta}, \ldots, \frac{z_{k}}{\gamma \tau+\delta}, \frac{\bar{z}_{k}}{\gamma \bar{\tau}+\delta} ; \frac{\alpha \tau+\beta}{\gamma \tau+\delta}, \frac{\alpha \bar{\tau}+\beta}{\gamma \bar{\tau}+\delta}\right)
\end{gathered}
$$

$(3.11)=m_{k}^{(1)}\left(u_{1}, \ldots, u_{k} ; z_{1}, \bar{z}_{1}, \ldots, z_{k}, \bar{z}_{k} ; \tau, \bar{\tau}\right)$.

A conformal full field algebra over $V^{L} \otimes V^{R}$ is said to be invariant under the action of (3.10) if for all $u_{1}, \ldots, u_{k} \in V$, (3.11) holds. If (3.6) is invariant under the action of all elements of $S L(2, \mathbb{Z})$, we say that (3.6) is modular invariant. When the function (3.6) is modular invariant, we call it the genus-one correlation function associated to $u_{1}, \ldots, u_{k} \in V$. A conformal full field algebra over $V^{L} \otimes V^{R}$ is said to be modular invariant if it is invariant under the action of all elements of $S L(2, \mathbb{Z})$.

Since the modular group $S L(2, \mathbb{Z})$ is generated by the elements

$$
S=\left(\begin{array}{cc}
0 & 1 \\
-1 & 0
\end{array}\right)
$$

and

$$
T=\left(\begin{array}{ll}
1 & 1 \\
0 & 1
\end{array}\right)
$$

to see whether a conformal full field algebra over $V^{L} \otimes V^{R}$ is modular invariant, we need only discuss the invariance under these two particular elements. 
For the element $T$, we have:

Proposition 3.6. A conformal full field algebra over $V^{L} \otimes V^{R}$ is invariant under the action of $T$ if and only if $c^{L} \equiv c^{R} \bmod 24$.

Proof. In the region $1>\left|e^{2 \pi i z_{1}}\right|, \ldots,\left|e^{2 \pi i z_{k}}\right|>\left|q_{\tau}\right|>0$ and $z_{i} \neq z_{j}$ for $i \neq j$, for $u_{1}, \ldots, u_{k} \in V$, replace $\tau$ and $\bar{\tau}$ in (3.5) by $\tau+1$ and $\bar{\tau}+1$, respectively. Then by the single-valuedness property (1.9), the only change is an overall constant factor $e^{2 \pi i \frac{c^{L}-c^{R}}{24}}$. Hence, we obtain, in this region,

$$
\begin{aligned}
& m_{k}^{(1)}\left(u_{1}, \ldots, u_{k} ; z_{1}, \bar{z}_{1}, \ldots, z_{k}, \bar{z}_{k} ; \tau+1, \bar{\tau}+1\right) \\
& \quad=m_{k}^{(1)}\left(u_{1}, \ldots, u_{k} ; z_{1}, \bar{z}_{1}, \ldots, z_{k}, \bar{z}_{k} ; \tau, \bar{\tau}\right) e^{2 \pi i \frac{c^{L}-c^{R}}{24}} .
\end{aligned}
$$

From (3.12), the meaning of the invariance of $F$ under $T$ and the fact that (3.6) are not all 0 (for example, it is not 0 when $u_{1}=\cdots=u_{k}=1$ ), we see that $F$ is invariant under $T$ if and only if $e^{2 \pi i \frac{c^{L}-c^{R}}{24}}=1$, or equivalently, $c^{L} \equiv c^{R} \bmod 24$.

The invariance under $S$ is the most important. We need to first introduce matrix elements associated to the actions of $S$ on the chiral genus-one correlation functions. For $a^{L}, a_{1}^{L} \in \mathcal{A}^{L}$, let $\left\{\mathcal{Y}_{a^{L} a_{1}^{L} ; i}^{a_{1}^{L} ;(1)} \mid i=1, \ldots, N_{a^{L} a_{1}^{L}}^{a^{L}}\right\}$ and $\left\{\mathcal{Y}_{a^{L} a_{1}^{L} ; i}^{a_{1}^{L} ;(2)} \mid i=1, \ldots, N_{a^{L} a_{1}^{L}}^{a^{L}}\right\}$ be bases of the spaces $\mathcal{V}_{a^{L} a_{1}^{L}}^{a_{1}^{L}}$ of intertwining operators of types $\left(\begin{array}{c}W^{a_{1}^{L}} \\ W^{a^{L}} W^{a_{1}^{L}}\end{array}\right)$ and for $a^{R}, a_{1}^{R} \in \mathcal{A}^{R}$, let $\left\{Y_{a^{R} a_{1}^{R} ; i}^{a_{1}^{R} ;(1)} \mid i=1, \ldots, N_{a^{R} a_{1}^{R}}^{a_{R}^{R}}\right\},\left\{Y_{a^{R} a_{1}^{R} ; i}^{a_{1}^{R} ;(2)} \mid i=1, \ldots, N_{a^{R} a_{1}^{R}}^{a_{R}^{R}}\right\}$ be bases of the space $\mathcal{V}_{a^{R} a_{1}^{R}}^{a_{1}^{R}}$ of intertwining operators of types $\left(\begin{array}{c}W^{a_{1}^{R}} \\ W^{a^{R}} W^{a_{1}^{R}}\end{array}\right)$. From Theorem 2.4. we know that for $a^{L} \in \mathcal{A}^{L}$, there exist $S\left(\mathcal{Y}_{a^{L} a_{1}^{L} ; j}^{a_{1}^{L} ;(1)} ; \mathcal{Y}_{a^{L} a_{2}^{L} ; j}^{a_{2}^{L} ;(2)}\right)$ for $a_{1}^{L}, a_{2}^{L} \in \mathcal{A}^{L}$, $i=1, \ldots, N_{a^{L} a_{1}^{L}}^{a^{L}}, j=1, \ldots, N_{a^{L} a_{2}^{L}}^{a^{L}}$ and for $a^{R} \in \mathcal{A}^{R}$, there exist $S\left(\mathcal{Y}_{a^{R} a_{1}^{R} ; i^{R}}^{a_{1}^{R}(1)} \mathcal{Y}_{a^{R} a_{2}^{R} ; j}^{a_{2}^{R} ;(2)}\right)$ for $a_{1}^{R}, a_{2}^{R} \in \mathcal{A}^{R}, i=1, \ldots, N_{a^{R} a_{1}^{R}}^{a^{R}}, j=1, \ldots, N_{a^{R}}^{a_{2}^{R}} a_{2}^{R}$, such that

$$
\begin{aligned}
& \left(\Phi\left(\mathcal{Y}_{a^{L} a_{1}^{L} ; i}^{a_{1}^{L} ;(1)}\right)\right)\left(\left(-\frac{1}{\tau}\right)^{L^{L}(0)} w_{a^{L}} ;-\frac{z}{\tau} ;-\frac{1}{\tau}\right) \\
& \quad=\sum_{a_{2}^{L} \in \mathcal{A}^{L}} \sum_{j=1}^{N_{a^{L}}^{a_{a}^{L}}} S\left(\mathcal{Y}_{a^{L} a_{1}^{L} ; i}^{a_{1}^{L} ;(1)} ; \mathcal{Y}_{a^{L} a_{2}^{L} ; j}^{a_{2}^{L} ;(2)}\right)\left(\Phi\left(\mathcal{Y}_{a^{L} a_{2}^{L} ; j}^{a_{2}^{L} ;(2)}\right)\right)\left(w_{a^{L}}, z ; \tau\right)
\end{aligned}
$$

for $w_{a^{L}} \in W^{a^{L}}$ and

$$
\begin{aligned}
& \left(\Phi\left(\mathcal{Y}_{a^{R} a_{1}^{R} ; i}^{a_{1}^{R} ;(1)}\right)\right)\left(\left(-\frac{1}{\tau}\right)^{L^{R}(0)} w_{a^{R}} ;-\frac{z}{\tau} ;-\frac{1}{\tau}\right) \\
& =\sum_{a_{2}^{R} \in \mathcal{A}^{R}} \sum_{j=1}^{\substack{N_{a^{R}}^{a_{2}^{R}} \\
a_{2}^{R}}} S\left(\mathcal{Y}_{a^{R} a_{1}^{R} ; i}^{a_{1}^{R} ;(1)} ; \mathcal{Y}_{a^{R} a_{2}^{R} ; j}^{a_{2}^{R} ;(2)}\right)\left(\Phi\left(\mathcal{Y}_{a_{2}^{R} a_{2}^{R} ; j}^{a_{R}^{R} ;(2)}\right)\right)\left(w_{a^{R}} ; z ; \tau\right) \text {. }
\end{aligned}
$$

We need the following. 
Lemma 3.7. For $w_{a^{R}} \in W^{a^{R}}$,

$$
\begin{aligned}
& \left(\Phi\left(\mathcal{Y}_{a^{R} a_{1}^{R} ; i}^{a_{1}^{R} ;(1)}\right)\left(e^{-\pi i L^{R}(0)}\left(-\frac{1}{\bar{\tau}}\right)^{L^{R}(0)} w_{a} ; \frac{\bar{z}}{\bar{\tau}} ; \frac{1}{\bar{\tau}}\right)\right. \\
& (3.15)=\sum_{a_{2}^{R} \in \mathcal{A}^{R}} \sum_{j=1}^{N_{a_{2}^{R}}^{a_{2}^{R}}} S^{-1}\left(\mathcal{Y}_{a^{R} a_{1}^{R} ; i}^{a_{1}^{R} ;(1)} ; \mathcal{Y}_{a^{R} a_{2}^{R} ; j}^{a_{2}^{R} ;(2)}\right)\left(\Phi\left(\mathcal{Y}_{a^{R} a_{2}^{R} ; j}^{a_{2}^{R} ;(2)}\right)\right)\left(e^{-\pi i L^{R}(0)} w_{a^{R}} ;-\bar{z} ;-\bar{\tau}\right),
\end{aligned}
$$

where $S^{-1}\left(\mathcal{Y}_{a^{R} a_{1}^{R} ; i}^{a_{R}^{R} ;(1)} ; \mathcal{Y}_{a^{R} a_{2}^{R} ; j}^{a_{R}^{R} ;(2)}\right)$ are the matrix elements of the inverse of the action of $S$, that is,

$$
\sum_{a_{2}^{R} \in \mathcal{A}^{R}} \sum_{j=1}^{\substack{N_{a}^{a_{2}^{R}} \\ a_{a}{ }^{R}}} S^{-1}\left(\mathcal{Y}_{a^{R} a_{1}^{R} ; i}^{a_{1}^{R} ;(1)} ; \mathcal{Y}_{a^{R} a_{2}^{R} ; j}^{a_{2}^{R} ;(2)}\right) S\left(\mathcal{Y}_{a^{R} a_{2}^{R} ; j}^{a_{2}^{R} ;(2)} ; \mathcal{Y}_{a^{R} a_{3}^{R} ; k}^{a_{3}^{R} ;(1)}\right)=\delta_{a_{1}^{R} a_{3}^{R} \delta_{i k}}
$$

Proof. From ( $(3.14)$, we see that the action of $S$ maps $\left(\Phi\left(\mathcal{Y}_{a^{R} a_{1}^{R} ; j}^{a_{1}^{R} ;(1)}\right)\right)\left(w_{a^{R}} ; z ; \tau\right)$ to

$$
\left(\Phi\left(\mathcal{Y}_{a^{R} a_{1}^{R} ; i}^{a_{1}^{R} ;()}\right)\right)\left(\left(-\frac{1}{\tau}\right)^{L^{R}(0)} w_{a^{R}} ;-\frac{z}{\tau} ;-\frac{1}{\tau}\right)
$$

So the inverse of the action of $S$ maps $\Phi\left(\mathcal{Y}_{a^{R} a_{1}^{R} ; j}^{a_{R}^{R} ;(1)}\right)\left(w_{a^{R}} ; z ; \tau\right)$ to

$$
\left(\Phi\left(\mathcal{Y}_{a^{R} a_{1}^{R} ; i}^{a_{1}^{R} ;()}\right)\right)\left(e^{-\log \tau L^{R}(0)} w_{a^{R}} ; \frac{z}{\tau} ;-\frac{1}{\tau}\right)
$$

Thus we have

$$
\begin{aligned}
& \left(\Phi\left(\mathcal{Y}_{a^{R} a_{1}^{R} ; i}^{a_{1}^{R} ;(1)}\right)\right)\left(e^{-\log \tau L^{R}(0)} w_{a^{R}} ; \frac{z}{\tau} ;-\frac{1}{\tau}\right) \\
& \quad=\sum_{a_{2}^{R} \in \mathcal{A}^{R}} \sum_{j=1}^{N_{a_{2}^{R}}^{a_{2}^{R}}} S^{-1}\left(\mathcal{Y}_{a^{R} a_{1}^{R} ; i}^{a_{1}^{R} ;(1)} ; \mathcal{Y}_{a^{R} a_{2}^{R} ; j}^{a_{2}^{R} ;(2)}\right)\left(\Phi\left(\mathcal{Y}_{a^{R} a_{2}^{R} ; j}^{a_{2}^{R} ;(2)}\right)\right)\left(w_{a} ; z ; \tau\right) .
\end{aligned}
$$

Note that when $\tau$ is in the upper half-plane, so is $-\bar{\tau}$. So we can substitute $-\bar{\tau}$ for $\tau$ in (3.16) above. We also substitute $-\bar{z}$ for $z$ and $e^{-\pi i L^{R}(0)} w_{a^{R}}$ for $w_{a^{R}}$ in (3.16). We obtain

$$
\begin{aligned}
& \left(\Phi\left(\mathcal{Y}_{a^{R} a_{1}^{R} ; i}^{a^{R} ;(1)}\right)\right)\left(e^{-\log (-\bar{\tau}) L^{R}(0)} e^{-\pi i L^{R}(0)} w_{a} ; \frac{\bar{z}}{\bar{\tau}} ; \frac{1}{\bar{\tau}}\right) \\
& (3.17)=\sum_{a_{2}^{R} \in \mathcal{A}^{R}} \sum_{j=1}^{N_{a_{2}^{R}}^{a_{2}^{R}}} S^{-1}\left(\mathcal{Y}_{a^{R} a_{1}^{R} ; i}^{a_{1}^{R} ;(1)} ; \mathcal{Y}_{a^{R} a_{2}^{R} ; j}^{a_{2}^{R} ;(2)}\right)\left(\Phi\left(\mathcal{Y}_{a^{R} a_{2}^{R} ; j}^{a_{2}^{R} ;(2)}\right)\right)\left(e^{-\pi i L^{R}(0)} w_{a^{R}} ;-\bar{z} ;-\bar{\tau}\right) .
\end{aligned}
$$

Note that $e^{-\pi i L^{R}(0)}$ commutes with $e^{-\log (-\bar{\tau}) L^{R}(0)}$. Since $0<\arg \tau<\pi$, we have $\pi<\arg \bar{\tau}<2 \pi, 0<\arg -\bar{\tau}<\pi$ and $0<\arg -\frac{1}{\tau}<\pi$. So $\arg -\frac{1}{\tau}=\arg -\bar{\tau}$. Then 
by our convention, on $W^{a^{R}}$, we have

$$
\begin{aligned}
e^{-\log (-\bar{\tau}) L^{R}(0)} & =e^{(-\log |-\bar{\tau}|-i \arg (-\bar{\tau})) L^{R}(0)} \\
& =e^{\left(\log \left|-\frac{1}{\tau}\right|-i \arg \left(-\frac{1}{\tau}\right)\right) L^{R}(0)} \\
& =e^{\overline{\log \left(-\frac{1}{\tau}\right) L^{R}(0)}} \\
& =\left(-\frac{1}{\bar{\tau}}\right)^{L^{R}(0)} .
\end{aligned}
$$

Changing the order of $e^{-\log (-\bar{\tau}) L^{R}(0)}$ and $e^{-\pi i L^{R}(0)}$ and then using (3.18), we see that (3.17) gives (3.15).

We now have:

Theorem 3.8. A conformal full field algebra over $V^{L} \otimes V^{R}$ is invariant under the action of $S$ if and only if

$$
\begin{aligned}
& \sum_{n=1}^{N} \sum_{i=1}^{N_{r L}^{r^{L}(n)}} \sum_{j=1}^{r_{(m) r}(n)} d_{m n ; i j}^{N_{r R}^{r^{R}(n)}} \sum_{(m) r^{R}(n)}^{n ;(1,1)} S\left(\mathcal{Y}_{r^{L}(m) r^{L}(n) ; i}^{r^{L}(n) ;(1)} ; \mathcal{Y}_{r^{L}(m) a^{L} ; k}^{a^{L} ;(2)}\right) . \\
& \cdot S^{-1}\left(\mathcal{Y}_{r^{R}(m) r^{R}(n) ; j}^{r^{R}(n) ;(1)} ; \mathcal{Y}_{r^{R}(m) a^{R} ; l}^{a^{R}(2)}\right) \\
& =\sum_{p \in\left(r^{L}\right)^{-1}\left(a^{L}\right) \cap\left(r^{R}\right)^{-1}\left(a^{R}\right)} d_{m p ; k l}^{p ;(2,2)}
\end{aligned}
$$

for $m=1, \ldots, N, a^{L} \in \mathcal{A}^{L}, a^{R} \in \mathcal{A}^{R}, k=1, \ldots, N_{r^{L}(m) a^{L}}^{a^{L}}, l=1, \ldots, N_{r^{R}(m) a^{R}}^{a^{R}}$.

Proof. From the convergence property of the correlation function maps (see Definition 1.1), we see that all the functions of the form (3.6) are invariant under the action of $S$ if and only if all the functions of the form (3.6) with $k=1$ are invariant under the action of $S$. We now show that all the functions of the form (3.6) with $k=1$ are invariant under the action of $S$ if and only if (3.19) holds.

If (3.19) holds, then from (3.2), the definition of the $q-\bar{q}$-trace of a map from $F$ to $\bar{F},(3.13)$ and (3.15), we have

$$
\begin{gathered}
m_{1}^{(1)}\left(\left(-\frac{1}{\tau}\right)^{L^{L}(0)} w_{r^{L}(m)} \otimes\left(-\frac{1}{\bar{\tau}}\right)^{L^{R}(0)} w_{r^{R}(m)} ;-\frac{z}{\tau},-\frac{\bar{z}}{\bar{\tau}} ;-\frac{1}{\tau},-\frac{1}{\bar{\tau}}\right) \\
=\operatorname{Tr}_{F} \mathbb{Y}\left(\mathcal{U}^{L}\left(e^{-2 \pi i \frac{z}{\tau}}\right) \mathcal{U}^{R}\left(e^{2 \pi i \frac{\bar{z}}{\tau}}\right)\left(-\frac{1}{\tau}\right)^{L^{L}(0)} w_{r^{L}(m)}\right. \\
\left.\otimes\left(-\frac{1}{\bar{\tau}}\right)^{L^{R}(0)} w_{r^{R}(m)} ; e^{-2 \pi i \frac{z}{\tau}}, e^{2 \pi i \frac{\bar{z}}{\tau}}\right) q_{-\frac{1}{\tau}}^{L^{L}(0)-\frac{c^{L}}{24}} q_{\frac{1}{\bar{\tau}}}^{L^{R}(0)-\frac{c^{R}}{24}} \\
=\operatorname{Tr}_{F} \sum_{l, n=1}^{N} \sum_{i=1}^{N_{r L(m) r}^{r^{L}(l)} \sum_{j=1}^{N_{r}(l)} \sum_{j) r}^{r^{R}(n)}} d_{m n ; i j}^{l ;(1,1)} \cdot \\
\cdot\left(\mathcal{Y}_{r^{L}(m) r^{L}(n) ; i}^{r^{L}(l) ;(1)}\left(\mathcal{U}^{L}\left(e^{-2 \pi i \frac{z}{\tau}}\right)\left(-\frac{1}{\tau}\right)^{L^{L}(0)} w_{r^{L}(m)}, e^{-2 \pi i \frac{z}{\tau}}\right)\right.
\end{gathered}
$$




$$
\begin{aligned}
& \left.\otimes \mathcal{Y}_{r^{R}(m) r^{R}(n) ; j}^{r^{R}(l) ;(1)}\left(\mathcal{U}^{R}\left(e^{2 \pi i \frac{\bar{z}}{\tau}}\right) e^{-\pi i L^{R}(0)}\left(-\frac{1}{\bar{\tau}}\right)^{L^{R}(0)} w_{r^{R}(m)}, e^{2 \pi i \frac{\bar{z}}{\tau}}\right)\right) \\
& \cdot q_{-\frac{1}{\tau}}^{L^{L}(0)-\frac{c^{L}}{24}} q_{\frac{1}{\tau}}^{L^{R}(0)-\frac{c^{R}}{24}} \\
& =\sum_{n=1}^{N} \sum_{i=1}^{N_{r L}^{r^{L}(l)}} \sum_{j=1}^{N_{(m) r}^{L}(n)} d_{m n ; i j}^{n ;(1,1)}\left(\left(\operatorname{Tr}_{W^{r^{L}(n)}} \mathcal{Y}_{r^{L}(m) r^{L}(n) ; i}^{r^{L}(n) ;(1)}\right.\right. \\
& \left.\left(\mathcal{U}^{L}\left(e^{-2 \pi i \frac{z}{\tau}}\right)\left(-\frac{1}{\tau}\right)^{L^{L}(0)} w_{r^{L}(m)}, e^{-2 \pi i \frac{z}{\tau}}\right) q_{-\frac{1}{\tau}}^{L^{L}(0)-\frac{c^{L}}{24}}\right) \\
& \otimes\left(\operatorname{Tr}_{W^{r^{R}(n)}} \mathcal{Y}_{r^{R}(m) r^{R}(n) ; j}^{r^{R}(n)(1)}\right. \\
& \left.\left.\left(\mathcal{U}^{R}\left(e^{2 \pi i \frac{\overline{\bar{\tau}}}{\bar{\tau}}}\right) e^{-\pi i L^{R}(0)}\left(-\frac{1}{\bar{\tau}}\right)^{L^{R}(0)} w_{r^{R}(m)}, e^{2 \pi i \frac{\bar{z}}{\bar{\tau}}}\right) \cdot q_{\frac{1}{\bar{\tau}}}^{L^{R}(0)-\frac{c^{R}}{24}}\right)\right) \\
& =\sum_{n=1}^{N} \sum_{i=1}^{N_{r}^{r_{(}^{L}(l)}} \sum_{j=1}^{N_{(m) r}^{L}(n)} d_{m n ; i j}^{N_{r}^{R}(l)} \\
& \cdot\left(\left(\Phi\left(\mathcal{Y}_{r^{L}(m) r^{L}(n) ; i}^{r^{L}(n) ;(1)}\right)\right)\left(\left(-\frac{1}{\tau}\right)^{L^{L}(0)} w_{r^{L}(m)} ;-\frac{z}{\tau} ;-\frac{1}{\tau}\right)\right. \\
& \otimes\left(\Phi\left(\mathcal{Y}_{r^{R}(m) r^{R}(n) ; j}^{r^{R}(n) ;(1)}\right)\left(e^{-\pi i L^{R}(0)}\left(-\frac{1}{\bar{\tau}}\right)^{L^{R}(0)} w_{r^{R}(m)} ; \frac{\bar{z}}{\bar{\tau}} ; \frac{1}{\bar{\tau}}\right)\right)
\end{aligned}
$$

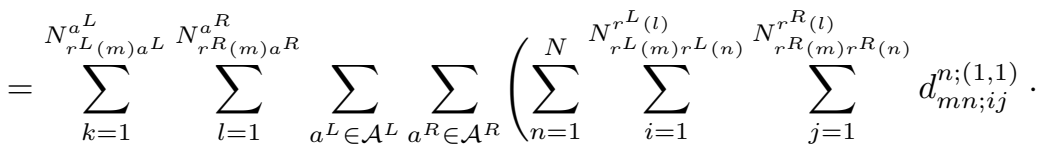

$$
\begin{aligned}
& \left.\cdot S\left(\mathcal{Y}_{r^{L}(m) r^{L}(n) ; i}^{r^{L}(n) ;(1)} ; \mathcal{Y}_{r^{L}(m) a^{L} ; k}^{a^{L} ;(2)}\right) S^{-1}\left(\mathcal{Y}_{r^{R}(m) r^{R}(n) ; j}^{r^{R}(n) ;(1)} ; \mathcal{Y}_{r^{R}(m) a^{R} ; l}^{a^{R} ;(2)}\right)\right) . \\
& \cdot\left(\left(\left(\Phi\left(\mathcal{Y}_{r^{L}(m) a^{L} ; k}^{a^{L} ;(2)}\right)\right)\left(w_{r^{L}(m)} ; z ; \tau\right)\right)\right. \\
& \otimes\left(\left(\Phi\left(\mathcal{Y}_{r^{R}(m) a^{R} ; l}^{a^{R} ;(2)}\right)\right)\left(e^{-\pi i L^{R}(0)} w_{r^{R}(m)} ;-\bar{z} ;-\bar{\tau}\right)\right)
\end{aligned}
$$

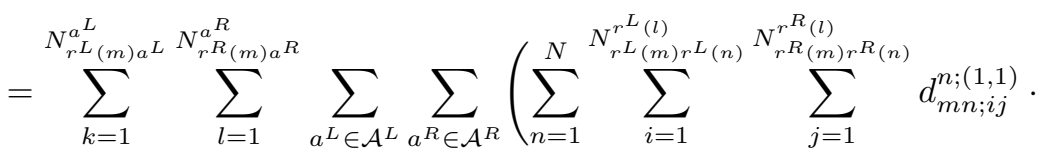

$$
\begin{aligned}
& \left.\cdot S\left(\mathcal{Y}_{r^{L}(m) r^{L}(n) ; i}^{r^{L}(n) ;(1)} ; \mathcal{Y}_{r^{L}(m) a^{L} ; k}^{a^{L} ;(2)}\right) S^{-1}\left(\mathcal{Y}_{r^{R}(m) r^{R}(n) ; j}^{r^{R}(n) ;(1)} ; \mathcal{Y}_{r^{R}(m) a^{R} ; l}^{a^{R} ;(2)}\right)\right) . \\
& \cdot\left(\left(\operatorname{Tr}_{W^{\alpha}{ }^{L}} \mathcal{Y}_{r^{L}(m) a^{L} ; k}^{a^{L} ;(2)}\left(\mathcal{U}^{L}\left(e^{2 \pi i z}\right) w_{r^{L}(m)}, e^{2 \pi i z}\right) q_{\tau}^{L^{L}(0)-\frac{c^{L}}{24}}\right)\right. \\
& \left.\otimes\left(\operatorname{Tr}_{W^{a} R} \mathcal{Y}_{r^{R}(m) a^{R} ; l}^{a^{R} ;(2)}\left(\mathcal{U}^{R}\left(e^{-2 \pi i \bar{z}}\right) e^{-\pi i L^{R}(0)} w_{r^{R}(m)}, e^{-2 \pi i \bar{z}}\right) q_{-\bar{\tau}}^{L^{R}(0)-\frac{c^{R}}{24}}\right)\right)
\end{aligned}
$$




$$
\begin{aligned}
& =\sum_{k=1}^{N_{r^{L}(m) a} a^{L}} \sum_{l=1}^{N_{r R(m) a^{R}}^{a^{R}}} \sum_{a^{L} \in \mathcal{A}^{L}} \sum_{a^{R} \in \mathcal{A}^{R}} \sum_{p \in\left(r^{L}\right)^{-1}} \sum_{\left(a^{L}\right) \cap\left(r^{R}\right)^{-1}\left(a^{R}\right)} d_{m p ; k l}^{p ;(2,2)} . \\
& \cdot\left(\left(\operatorname{Tr}_{W^{a}{ }^{L}} \mathcal{Y}_{r^{L}(m) a^{L} ; k}^{a^{L} ;(2)}\left(\mathcal{U}^{L}\left(e^{2 \pi i z}\right) w_{r^{L}(m)}, e^{2 \pi i z}\right) q_{\tau}^{L^{L}(0)-\frac{c^{L}}{24}}\right)\right. \\
& \left.\otimes\left(\operatorname{Tr}_{W^{a}{ }^{R}} \mathcal{Y}_{r^{R}(m) a^{R} ; l}^{a^{R} ;(2)}\left(\mathcal{U}^{R}\left(e^{-2 \pi i \bar{z}}\right) e^{-\pi i L^{R}(0)} w_{r^{R}(m)}, e^{-2 \pi i \bar{z}}\right) q_{-\bar{\tau}}^{L^{R}(0)-\frac{c^{R}}{24}}\right)\right) \\
& =\sum_{k=1}^{N_{r L(m) a}^{a^{L}}} \sum_{l=1}^{N_{r}^{a^{R}}} \sum_{p=1}^{a_{(m) a} R} d_{m p ; k l}^{p} . \\
& \cdot\left(\left(\operatorname{Tr}_{W^{a} L} \mathcal{Y}_{r^{L}(m) r^{L}(p) ; k}^{r^{L}(p)(2)}\left(\mathcal{U}^{L}\left(e^{2 \pi i z}\right) w_{r^{L}(m)}, e^{2 \pi i z}\right) q_{\tau}^{L^{L}(0)-\frac{c^{L}}{24}}\right)\right. \\
& \left.\otimes\left(\operatorname{Tr}_{W^{a}{ }^{R}} \mathcal{Y}_{r^{R}(m) r^{R}(p) ; l}^{r^{R}(p) ;(2)}\left(\mathcal{U}^{R}\left(e^{-2 \pi i \bar{z}}\right) e^{-\pi i L^{R}(0)} w_{r^{R}(m)}, e^{-2 \pi i \bar{z}}\right) q_{-\bar{\tau}}^{L^{R}(0)-\frac{c^{R}}{24}}\right)\right) \\
& =m_{1}^{(1)}\left(w_{r^{L}(m)} \otimes w_{r^{R}(m)} ; z, \bar{z} ; \tau, \bar{\tau}\right) .
\end{aligned}
$$

Conversely, the calculation (3.20) also shows that if $F$ is modular invariant, then (3.19) holds.

\section{S-Matrices ASSOCiated to IRREDUCiBle MODUleS AND MODULAR INVARIANCE}

We assume that $V$ is a vertex operator algebra satisfying Conditions 1.3, 1.4 and 1.5. We shall continue to use the notation in the preceding sections, in particular, that in Section 2.

For $a_{1}, a_{2} \in \mathcal{A}$ and $\mathcal{Y}$ an intertwining operator of type $\left(\begin{array}{c}W^{a_{1}} \\ W^{a_{2}} W^{a_{1}}\end{array}\right)$, we consider

$$
\begin{aligned}
\Psi(\mathcal{Y}): \coprod_{a \in \mathcal{A}} W^{a} & \rightarrow \mathbb{G}_{1 ; 1} \\
w_{a} & \mapsto \Psi(\mathcal{Y})\left(w_{a} ; \tau\right)
\end{aligned}
$$

for $a \in \mathcal{A}$ and $w_{a} \in W^{a}$, where for $w_{a} \in W^{a}$,

$$
\Psi(\mathcal{Y})\left(w_{a} ; \tau\right)=0
$$

when $a \neq a_{2}$ and

$$
\begin{aligned}
\Psi(\mathcal{Y})\left(w_{a_{2}} ; \tau\right) & =\Phi(\mathcal{Y})\left(w_{a_{2}} ; z ; \tau\right) \\
& =E\left(\operatorname{Tr}_{W^{a_{1}}} \mathcal{Y}\left(\mathcal{U}\left(e^{2 \pi i z}\right) w_{a_{2}}, e^{2 \pi i z}\right) q_{\tau}^{L(0)-\frac{c}{24}}\right)
\end{aligned}
$$

when $a=a_{2}$, and $\mathbb{G}_{1 ; 1}$ is the space spanned by functions of $\tau$ of the form $\Psi(\mathcal{Y})\left(w_{a} ; \tau\right)$ for $a, a_{1}, a_{2} \in \mathcal{A}$ and $\mathcal{Y}$ an intertwining operator of type $\left(\begin{array}{c}W^{a_{1}} \\ W^{a_{2}} W^{a_{1}}\end{array}\right)$. Here we use the notation $\Psi(\mathcal{Y})\left(w_{a} ; \tau\right)$ instead of $\Psi(\mathcal{Y})\left(w_{a} ; z ; \tau\right)$, because it was shown in the 
proof of Theorem 7.3 in [H7 that $\Psi(\mathcal{Y})\left(w_{a_{2}} ; \tau\right)$ is indeed independent of $z$. Let $\mathcal{F}_{1 ; 1}$ be the space of all maps of the form $\Psi(\mathcal{Y})$ for $a_{1}, a_{2} \in \mathcal{A}$ and $\mathcal{Y}$ an intertwining operator of type $\left(\begin{array}{c}W^{a_{1}} \\ W^{a_{2}} W^{a_{1}}\end{array}\right)$.

Let $\mathbb{G}_{1 ; 2}$ be the space of all single-valued analytic functions on the universal covering $\widetilde{M}_{1}^{2}$ of

$$
M_{1}^{2}=\left\{\left(z_{1}, z_{2}, \tau\right) \in \mathbb{C}^{3} \mid z_{1} \neq z_{2}+p \tau+q \text { for } p, q \in \mathbb{Z}, \tau \in \mathbb{H}\right\}
$$

spanned by functions of the form

$$
\left(\Phi\left(\mathcal{Y}_{1} \otimes \mathcal{Y}_{2}\right)\right)\left(w_{a_{1}}, w_{a_{2}} ; z_{1}, z_{2} ; \tau\right)
$$

for $a_{1}, a_{2}, a_{3}, a_{4} \in \mathcal{A}, w_{a_{1}} \in W^{a_{1}}, w_{a_{2}} \in W^{a_{2}}$, and $\mathcal{Y}_{1}$ and $\mathcal{Y}_{2}$ intertwining operators of types $\left(\begin{array}{c}W^{a_{4}} \\ W^{a_{1}} W^{a_{3}}\end{array}\right)$ and $\left(\begin{array}{c}W^{a_{3}} \\ W^{a_{2}} W^{a_{4}}\end{array}\right)$, respectively. This space is also spanned by the analytic extensions

$$
E\left(\operatorname{Tr}_{W^{a_{6}}} \mathcal{Y}_{3}\left(\mathcal{U}\left(e^{2 \pi i z_{1}}\right) \mathcal{Y}_{4}\left(w_{a_{1}}, z_{1}-z_{2}\right) w_{a_{2}}, e^{2 \pi i z_{2}}\right) q_{\tau}^{L(0)-\frac{c}{24}}\right)
$$

to the region given by $\Im(\tau)>0, z_{1} \neq z_{2}+k \tau+l$ for $k, l \in \mathbb{Z}$, of functions of the form

$$
\operatorname{Tr}_{W^{a_{6}}} \mathcal{Y}_{3}\left(\mathcal{U}\left(e^{2 \pi i z_{1}}\right) \mathcal{Y}_{4}\left(w_{a_{1}}, z_{1}-z_{2}\right) w_{a_{2}}, e^{2 \pi i z_{2}}\right) q_{\tau}^{L(0)-\frac{c}{24}}
$$

for $a_{1}, a_{2}, a_{3}, a_{4} \in \mathcal{A}, w_{a_{1}} \in W^{a_{1}}, w_{a_{2}} \in W^{a_{2}}$, and $\mathcal{Y}_{3}$ and $\mathcal{Y}_{4}$ intertwining operators of types $\left(\begin{array}{c}W^{a_{4}} \\ W^{a} W^{a_{4}}\end{array}\right)$ and $\left(\begin{array}{c}W^{a} \\ W^{a_{1}} W^{a_{2}}\end{array}\right)$, respectively. For $a_{3} \in \mathcal{A}$, let $\mathbb{G}_{1 ; 2}^{a_{3}}$ be the space of all single-valued analytic functions on the universal covering $\widetilde{M}_{1}^{2}$ of $M_{1}^{2}$ spanned by functions of the form (4.1) for $a_{1}, a_{2}, a_{4} \in \mathcal{A}, w_{a_{1}} \in W^{a_{1}}, w_{a_{2}} \in W^{a_{2}}$, and $\mathcal{Y}_{3}$ and $\mathcal{Y}_{4}$ intertwining operators of types $\left(\begin{array}{c}W^{a_{4}} \\ W^{a_{3}} W^{a_{4}}\end{array}\right)$ and $\left(\begin{array}{c}W^{a_{3}} \\ W^{a_{1}} W^{a_{2}}\end{array}\right)$, respectively.

Now for $a_{1}, a_{2}, a_{3} \in \mathcal{A}$, let $\left\{\mathcal{Y}_{a_{1} a_{2} ; i}^{a_{3}(1)} \mid i=1, \ldots, N_{a_{1} a_{2}}^{a_{2}}\right\}$ and $\left\{\mathcal{Y}_{a_{1} a_{2} ; i}^{a_{3} ;(2)} \mid i=\right.$ $\left.1, \ldots, N_{a_{1} a_{2}}^{a_{2}}\right\}$ be bases of the space $\mathcal{V}_{a_{1} a_{2}}^{a_{3}}$ of intertwining operators of type $\left(\begin{array}{c}W^{a_{3}} \\ W^{a_{1}} W^{a_{2}}\end{array}\right)$. We need the following result proved in [H6]:

Proposition 4.1. For $a_{1}, a_{2} \in \mathcal{A}$, the maps from $W^{a_{1}} \otimes W^{a_{2}}$ to $\mathbb{G}_{1 ; 2}$ given by

$$
\begin{aligned}
& w_{a_{1}} \otimes w_{a_{2}} \mapsto \\
& \quad E\left(\operatorname{Tr}_{W^{a_{4}}} \mathcal{Y}_{a_{1} a_{3} ; i}^{a_{4} ;(1)}\left(\mathcal{U}\left(e^{2 \pi i z_{1}}\right) w_{a_{1}}, e^{2 \pi i z_{1}}\right) \mathcal{Y}_{a_{2} a_{4} ; j}^{a_{3} ;(2)}\left(\mathcal{U}\left(e^{2 \pi i z_{2}}\right) w_{a_{2}}, e^{2 \pi i z_{2}}\right) q_{\tau}^{L(0)-\frac{c}{24}}\right),
\end{aligned}
$$

$a_{3}, a_{4} \in \mathcal{A}, i=1, \ldots, N_{a_{1} a_{3}}^{a_{4}}, j=1, \ldots, N_{a_{2} a_{4}}^{a_{3}}$, are linearly independent. Similarly, for $a_{1}, a_{2} \in \mathcal{A}$, the maps from $W^{a_{1}} \otimes W^{a_{2}}$ to $\mathbb{G}_{1 ; 2}$ given by

$$
w_{a_{1}} \otimes w_{a_{2}} \mapsto E\left(\operatorname{Tr}_{W^{a_{4}}} \mathcal{Y}_{a_{3} a_{4} ; k}^{a_{4} ;(3)}\left(\mathcal{U}\left(e^{2 \pi i z_{2}}\right) \mathcal{Y}_{a_{1} a_{2} ; l}^{a_{3} ;(4)}\left(w_{a_{1}}, z_{1}-z_{2}\right) w_{a_{2}}, e^{2 \pi i z_{2}}\right) q_{\tau}^{L(0)-\frac{c}{24}}\right),
$$

$a_{3}, a_{4} \in \mathcal{A}, k=1, \ldots, N_{a_{3} a_{4}}^{a_{3}}, l=1, \ldots, N_{a_{1} a_{2}}^{a_{3}}$, are linearly independent. 
For $a \in \mathcal{A}$ and intertwining operators $\mathcal{Y}_{1}$ and $\mathcal{Y}_{2}$ of types for intertwining operators of types $\left(\begin{array}{c}W^{a_{1}} \\ W^{a} W^{a_{1}}\end{array}\right)$ and $\left(\begin{array}{c}W^{a} \\ W^{a_{2}} W^{a_{2}^{\prime}}\end{array}\right)$, respectively, we consider the maps

$$
\begin{aligned}
\Psi\left(\mathcal{Y}_{1} \otimes \mathcal{Y}_{2}\right): \coprod_{a_{3} \in \mathcal{A}} W^{a_{3}} \otimes W^{a_{3}^{\prime}} & \rightarrow \mathbb{G}_{1 ; 2} \\
w_{a_{3}} \otimes w_{a_{3}^{\prime}} & \mapsto\left(\Psi\left(\mathcal{Y}_{1} \otimes \mathcal{Y}_{2}\right)\right)\left(w_{a_{3}} \otimes w_{a_{3}^{\prime}} ; z_{1}, z_{2} ; \tau\right)
\end{aligned}
$$

for $a_{1}, a_{2} \in \mathcal{A}, i=1, \ldots, N_{a a_{1}}^{a_{1}}, j=1, \ldots, N_{a_{2} a_{2}^{\prime}}^{a}$, where

$$
\left(\Psi\left(\mathcal{Y}_{1} \otimes \mathcal{Y}_{2}\right)\right)\left(w_{a_{3}} \otimes w_{a_{3}^{\prime}} ; z_{1}, z_{2} ; \tau\right)=0
$$

when $a_{3} \neq a_{2}$ and

$$
\begin{aligned}
& \left(\Psi\left(\mathcal{Y}_{1} \otimes \mathcal{Y}_{2}\right)\right)\left(w_{a_{3}} \otimes w_{a_{3}^{\prime}} ; z_{1}, z_{2} ; \tau\right) \\
& \quad=E\left(\operatorname{Tr}_{W^{a_{1}}} \mathcal{Y}_{1}\left(\mathcal{U}\left(e^{2 \pi i z_{2}}\right) \mathcal{Y}_{2}\left(w_{a_{2}}, z_{1}-z_{2}\right) w_{a_{2}^{\prime}}, e^{2 \pi i z_{2}}\right) q_{\tau}^{L(0)-\frac{c}{24}}\right) .
\end{aligned}
$$

For $a \in \mathcal{A}$, let $\mathcal{F}_{1 ; 2}^{a}$ be the space spanned by linear maps of the form $\Psi\left(\mathcal{Y}_{1} \otimes \mathcal{Y}_{2}\right)$ for $a_{1}, a_{2} \in \mathcal{A}$ and for intertwining operators $\mathcal{Y}_{1}$ and $\mathcal{Y}_{2}$ of types $\left(\begin{array}{c}W^{a_{1}} \\ W^{a} W^{a_{1}}\end{array}\right)$ and $\left(\begin{array}{c}W^{a} \\ W^{a_{2}} W^{a_{2}^{\prime}}\end{array}\right)$, respectively. For $a \in \mathcal{A}$, we also let $\mathcal{F}_{1 ; 2}^{n a}$ be the space spanned by linear maps of the form $\Psi\left(\mathcal{Y}_{1} \otimes \mathcal{Y}_{2}\right)$ for $a_{1}, a_{2}, a_{3} \in \mathcal{A}, a_{3} \neq a$, and for intertwining operators $\mathcal{Y}_{1}$ and $\mathcal{Y}_{2}$ of types $\left(\begin{array}{c}W^{a_{1}} \\ W^{a_{3}} W^{a_{1}}\end{array}\right)$ and $\left(\begin{array}{c}W^{a_{3}} \\ W^{a_{2}} W^{a_{2}^{\prime}}\end{array}\right)$, respectively. Let $\mathcal{F}_{1 ; 2}$ be the sum of $\mathcal{F}_{1 ; 2}^{a}$ for $a \in \mathcal{A}$. We have:

Proposition 4.2. For $a \in \mathcal{A}$, the intersection of $\mathcal{F}_{1 ; 2}^{a}$ and $\mathcal{F}_{1 ; 2}^{n a}$ is 0 . In particular,

$$
\mathcal{F}_{1 ; 2}=\mathcal{F}_{1 ; 2}^{a} \oplus \mathcal{F}_{1 ; 2}^{n a}
$$

and there exists a projection $\pi: \mathcal{F}_{1 ; 2} \rightarrow \mathcal{F}_{1 ; 2}^{a}$.

Proof. By Proposition 4.1 $\Psi\left(\mathcal{Y}_{a a_{1} ; k}^{a_{1} ;(1)} \otimes \mathcal{Y}_{a_{2} a_{2}^{\prime} ;}^{\left.a_{3} ; 2\right)}\right.$ for $a_{1}, a_{2}, a_{3} \in \mathcal{A}, k=1, \ldots, N_{a_{3} a_{1}}^{a_{1}}$ and $l=1, \ldots, N_{a_{2} a_{2}^{\prime}}^{a_{3}}$ are linearly independent. Thus the intersection of the space spanned by $\Psi\left(\mathcal{Y}_{a a_{1} ; k}^{a_{1} ;()} \otimes \mathcal{Y}_{a_{2} a_{2}^{\prime} ; l}^{a ;()}\right)$ for $a_{1}, a_{2} \in \mathcal{A}, k=1, \ldots, N_{a a_{1}}^{a_{1}}, l=1, \ldots, N_{a_{2} a_{2}^{\prime}}^{a}$, and the space spanned by $\Psi\left(\mathcal{Y}_{a_{3} a_{1} ; k}^{a_{1} ;(1)} \otimes \mathcal{Y}_{a_{2} a_{2}^{\prime} ; l}^{a_{3} ;(2)}\right)$ for $a_{1}, a_{2}, a_{3} \in \mathcal{A}, a_{3} \neq a, k=1, \ldots, N_{a_{3} a_{1}}^{a_{1}}$ and $l=1, \ldots, N_{a_{2} a_{2}^{\prime}}^{a_{3}}$ are 0 .

In the remaining part of this section, $\left\{\mathcal{Y}_{a_{1} a_{2} ; i}^{a_{3} ;(p)} \mid i=1, \ldots, N_{a_{1} a_{2}}^{a_{3}}\right\}$ for $p=$ $1,2,3,4,5,6$ are bases of the spaces of intertwining operators of type $\left(\begin{array}{c}W^{a_{3}} \\ W^{a_{1}} W^{a_{2}}\end{array}\right)$, $a_{1}, a_{2}, a_{3} \in \mathcal{A}$.

We have the following lemma, which is a generalization of Lemma 4.2 in [H10]. 
Lemma 4.3. For $a_{1}, a_{2}, a \in \mathcal{A}, w_{a_{2}} \in W^{a_{2}}$ and $w_{a_{2}^{\prime}} \in W^{a_{2}^{\prime}}$, we have

$$
\begin{aligned}
& \left(\Psi\left(\mathcal{Y}_{a a_{1} ; p}^{a_{1} ;(1)} \otimes \mathcal{Y}_{a_{2} a_{2}^{\prime} ; q}^{a ;(2)}\right)\right)\left(w_{a_{2}} \otimes w_{a_{2}^{\prime}} ; z_{1}, z_{2}-1 ; \tau\right) \\
& =\sum_{a \in \mathcal{A}} \sum_{i=1}^{N_{a_{2} a_{3}}^{a_{1}}} \sum_{j=1}^{N_{a_{2}^{\prime} a_{1}}^{a_{a_{3}}}} \sum_{a_{4} \in \mathcal{A}} \sum_{k=1}^{N_{a_{4} a_{1}}^{a_{1} a_{1}}} \sum_{l=1}^{N_{a_{2} a_{2}^{\prime}}^{a_{4}}} e^{-2 \pi i\left(h_{a_{3}}-h_{a_{1}}\right)} . \\
& \cdot F^{-1}\left(\mathcal{Y}_{a a_{1} ; p}^{a_{1} ;(1)} \otimes \mathcal{Y}_{a_{2} a_{2}^{\prime} ; q}^{a ;(2)} ; \mathcal{Y}_{a_{2} a_{3} ; i}^{a_{1} ;(3)} \otimes \mathcal{Y}_{a_{2}^{\prime} a_{1} ; j}^{a_{3} ;(4)}\right) \text {. } \\
& \cdot F\left(\mathcal{Y}_{a_{2} a_{3} ; i}^{a_{1} ;(3)} \otimes \mathcal{Y}_{a_{2}^{\prime} a_{1} ; j}^{a_{3} ;(4)} ; \mathcal{Y}_{a_{4} a_{1} ; k}^{a_{1} ;(5)} \otimes \mathcal{Y}_{a_{2} a_{2}^{\prime} ; l}^{a_{4} ;(6)}\right) \cdot \\
& \cdot E\left(\operatorname { T r } _ { W ^ { a _ { 1 } } } \mathcal { Y } _ { a _ { 4 } a _ { 1 } ; k } ^ { a _ { 1 } ; ( 5 ) } \left(\mathcal{U}\left(e^{2 \pi i z_{2}}\right)\right.\right. \text {. } \\
& \left.\left.\cdot \mathcal{Y}_{a_{2} a_{2}^{\prime} ; l}^{a_{4} ;(6)}\left(w_{a_{2}}, z_{1}-z_{2}\right) w_{a_{2}^{\prime}}, e^{2 \pi i z_{2}}\right) q_{\tau}^{L(0)-\frac{c}{24}}\right)
\end{aligned}
$$

and

$$
\begin{aligned}
& \left(\Psi\left(\mathcal{Y}_{a a_{1} ; i}^{a_{1} ;(1)} \otimes \mathcal{Y}_{a_{2} a_{2}^{\prime} ; j}^{a ;(2)}\right)\right)\left(w_{a_{2}} \otimes w_{a_{2}^{\prime}} ; z_{1}, z_{2}+\tau ; \tau\right) \\
& =\sum_{a_{3} \in \mathcal{A}} \sum_{i=1}^{N_{a_{2}}^{a_{1} a_{3}}} \sum_{j=1}^{N_{a_{2}^{\prime} a_{1}}^{a_{3}}} \sum_{a_{4} \in \mathcal{A}} \sum_{k=1}^{N_{a_{4} a_{3}}^{a_{3}}} \sum_{l=1}^{N_{a_{2} a_{2}}^{a_{4}}} e^{\pi i\left(-2 h_{a_{2}}+h_{a_{4}}\right) .} \\
& \cdot F^{-1}\left(\mathcal{Y}_{a a_{1} ; p}^{a_{1} ;(p)} \otimes \mathcal{Y}_{a_{2} a_{2}^{\prime} ; j}^{a ;(2)} ; \sigma_{23}\left(\mathcal{Y}_{a_{2} a_{1}^{\prime} ; i}^{\left.a_{3}^{\prime} ; 3\right)} \otimes \sigma_{13}\left(\mathcal{Y}_{a_{3}^{\prime} a_{1} ; j}^{a_{2} ;(4)}\right)\right)\right. \text {. } \\
& \cdot F\left(\mathcal{Y}_{a_{2} a_{1}^{\prime} ; i}^{a_{3}^{\prime} ;(3)} \otimes \sigma_{123}\left(\mathcal{Y}_{a_{3}^{\prime} a_{1} ; j}^{a_{2} ;(4)}\right) ; \mathcal{Y}_{a_{4} a_{3}^{\prime} ; k}^{a_{3}^{\prime} ;(5)} \otimes \mathcal{Y}_{a_{2} a_{2}^{\prime} ; l}^{a_{4} ;(6)}\right) \text {. } \\
& \cdot E\left(\operatorname{Tr}_{W^{a_{3}}} \mathcal{Y}_{a_{4} a_{3} ; k}^{a_{3} ;(5)}\left(\mathcal{U}\left(e^{2 \pi i z_{2}}\right) \mathcal{Y}_{a_{2} a_{2}^{\prime} ; l}^{a_{4} ;(6)}\left(w_{a_{2}}, z_{1}-z_{2}\right) w_{a_{2}^{\prime}}, e^{2 \pi i z_{2}}\right) q_{\tau}^{L(0)-\frac{c}{24}}\right) .
\end{aligned}
$$

In particular, for any $a_{1}, a_{2} \in \mathcal{A}$, the maps from $\coprod_{a_{3} \in \mathcal{A}} W^{a_{3}} \otimes W^{a_{3}^{\prime}}$ to the space of single-valued analytic functions on $\widetilde{M}_{1}^{2}$ given by

$$
\begin{aligned}
& w_{a_{3}} \otimes w_{a_{3}^{\prime}} \mapsto\left(\Psi\left(\mathcal{Y}_{a a_{1} ; p}^{a_{1} ;(1)} \otimes \mathcal{Y}_{a_{2} a_{2}^{\prime} ; q}^{a ;(2)}\right)\right)\left(w_{a_{3}} \otimes w_{a_{3}^{\prime}} ; z_{1}, z_{2}-1 ; \tau\right), \\
& w_{a_{3}} \otimes w_{a_{3}^{\prime}} \mapsto\left(\Psi\left(\mathcal{Y}_{a a_{1} ; i}^{\left.a_{1} ; 1\right)} \otimes \mathcal{Y}_{a_{2} a_{2}^{\prime} ; j}^{a ;(2)}\right)\left(w_{a_{3}} \otimes w_{a_{3}^{\prime}} ; z_{1}, z_{2}+\tau ; \tau\right)\right. \\
& \text { for } w_{a_{3}} \in W^{a_{3}} \text { and } w_{a_{3}^{\prime}} \in W^{a_{3}^{\prime}} \text { are in } \mathcal{F}_{1 ; 2} .
\end{aligned}
$$

Proof. Using the definition of $\Psi\left(\mathcal{Y}_{a a_{1} ; p}^{a_{1} ;(1)} \otimes \mathcal{Y}_{a_{2} a_{2}^{\prime} ; q}^{a ;(2)}\right)$, the genus-one associativity properties ((2.9) and (2.10) in [H10]) and

$$
\mathcal{Y}\left(w_{a_{1}}, x\right) w_{a_{2}} \in x^{\Delta(\mathcal{Y})} W^{a_{3}}\left[\left[x, x^{-1}\right]\right],
$$

where

we obtain

$$
\Delta(\mathcal{Y})=h_{a_{3}}-h_{a_{1}}-h_{a_{2}},
$$

$$
\begin{aligned}
& \left(\Psi\left(\mathcal{Y}_{a a_{1} ; p}^{a_{1} ;(1)} \otimes \mathcal{Y}_{a_{2} a_{2}^{\prime} ; q}^{a ;(2)}\right)\right)\left(w_{a_{2}} \otimes w_{a_{2}^{\prime}} ; z_{1}, z_{2}-1 ; \tau\right) \\
& =E\left(\operatorname { T r } _ { W ^ { a _ { 1 } } } \mathcal { Y } _ { a a _ { 1 } ; p } ^ { a _ { 1 } } \left(\mathcal{U}\left(e^{2 \pi i\left(z_{2}-1\right)}\right) \cdot\right.\right. \\
& \left.\left.\quad \mathcal{Y}_{a_{2} a_{2}^{\prime} ; q}^{a}\left(w_{a_{2}}, z_{1}-\left(z_{2}-1\right)\right) w_{a_{2}^{\prime}}, e^{2 \pi i\left(z_{2}-1\right)}\right) q_{\tau}^{L(0)-\frac{c}{24}}\right)
\end{aligned}
$$




$$
\begin{aligned}
& =\sum_{a_{3} \in \mathcal{A}} \sum_{i=1}^{N_{a_{2} a_{3}}^{a_{1}}} \sum_{j=1}^{N_{a_{2}^{\prime} a_{1}}^{a_{3}}} F^{-1}\left(\mathcal{Y}_{a a_{1} ; p}^{a_{1} ;(1)} \otimes \mathcal{Y}_{a_{2} a_{2}^{\prime} ; q}^{a ;(2)} ; \mathcal{Y}_{a_{2} a_{3} ; i}^{a_{1} ;(3)} \otimes \mathcal{Y}_{a_{2}^{\prime} a_{1} ; j}^{a_{3} ;(4)}\right) \\
& \cdot E\left(\operatorname{Tr}_{W^{a_{1}}} \mathcal{Y}_{a_{2} a_{3} ; i}^{a_{1} ;(3)}\left(\mathcal{U}\left(e^{2 \pi i z_{1}}\right) w_{a_{2}}, e^{2 \pi i z_{1}}\right) \cdot\right. \\
& \left.\cdot \mathcal{Y}_{a_{2}^{\prime} a_{1} ; j}^{a_{3} ;(4)}\left(\mathcal{U}\left(e^{2 \pi i\left(z_{2}-1\right)}\right) w_{a_{2}^{\prime}}, e^{2 \pi i\left(z_{2}-1\right)}\right) q_{\tau}^{L(0)-\frac{c}{24}}\right) \\
& =\sum_{a_{3} \in \mathcal{A}} \sum_{i=1}^{N_{a_{2} a_{3}}^{a_{1}}} \sum_{j=1}^{N_{a_{2}^{\prime} a_{1}}^{a_{3}}} F^{-1}\left(\mathcal{Y}_{a a_{1} ; p}^{a_{1} ;(1)} \otimes \mathcal{Y}_{a_{2} a_{2}^{\prime} ; q^{\prime}}^{a ;(2)} ; \mathcal{Y}_{a_{2} a_{3} ; i}^{a_{1} ;(3)} \otimes \mathcal{Y}_{a_{2}^{\prime} a_{1} ; j}^{a_{3} ;(4)}\right) \\
& \cdot E\left(\operatorname{Tr}_{W^{a_{1}}} \mathcal{Y}_{a_{2} a_{3} ; i}^{a_{1} ;(3)}\left(\mathcal{U}\left(e^{2 \pi i z_{1}}\right) w_{a_{2}}, e^{2 \pi i z_{1}}\right) \cdot\right. \\
& \left.\cdot \mathcal{Y}_{a_{2}^{\prime} a_{1} ; j}^{a_{3} ;(4)}\left(\mathcal{U}\left(e^{-2 \pi i} e^{2 \pi i z_{2}}\right) w_{a_{2}^{\prime}}, e^{-2 \pi i} e^{2 \pi i z_{2}}\right) q_{\tau}^{L(0)-\frac{c}{24}}\right)
\end{aligned}
$$

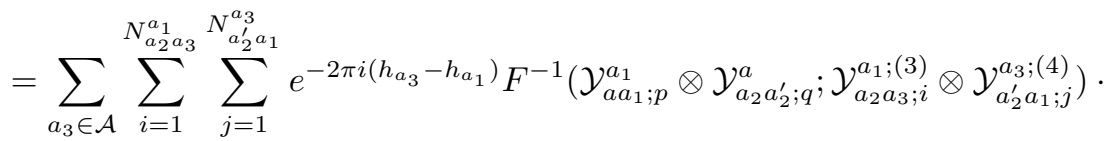

$$
\begin{aligned}
& \cdot E\left(\operatorname{Tr}_{W^{a_{1}}} \mathcal{Y}_{a_{2} a_{3} ; i}^{a_{1} ;(3)}\left(\mathcal{U}\left(e^{2 \pi i z_{1}}\right) w_{a_{2}}, e^{2 \pi i z_{1}}\right) \cdot\right. \\
& \left.\cdot \mathcal{Y}_{a_{2}^{\prime} a_{1} ; j}^{a_{3} ;(4)}\left(\mathcal{U}\left(e^{2 \pi i z_{2}}\right) w_{a_{2}^{\prime}}, e^{2 \pi i z_{2}}\right) q_{\tau}^{L(0)-\frac{c}{24}}\right)
\end{aligned}
$$

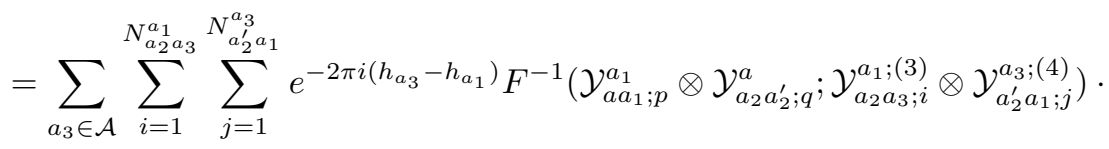

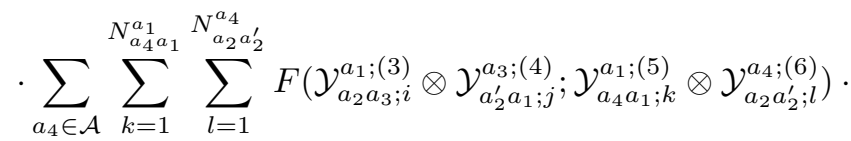

$$
\begin{aligned}
& \cdot E\left(\operatorname { T r } _ { W ^ { a _ { 1 } } } \mathcal { Y } _ { a _ { 4 } a _ { 1 } ; k } ^ { a _ { 1 } ; ( 5 ) } \left(\mathcal{U}\left(e^{2 \pi i z_{2}}\right) \cdot\right.\right. \\
& \left.\left.\cdot \mathcal{Y}_{a_{2} a_{2}^{\prime} ; l}^{a_{4} ;(6)}\left(w_{a_{2}}, z_{1}-z_{2}\right) w_{a_{2}^{\prime}}, e^{2 \pi i z_{2}}\right) q_{\tau}^{L(0)-\frac{c}{24}}\right),
\end{aligned}
$$

proving (4.2).

The proof of (4.3) is more complicated. Using the definition of $\Psi\left(\mathcal{Y}_{a a_{1} ; p}^{a_{1} ;(1)} \otimes\right.$ $\left.\mathcal{Y}_{a_{2} a_{2}^{\prime} ; q}^{a ;(2)}\right)$, the genus-one associativity properties $((2.9)$ and $(2.10)$ in [H10]), the $L(0)$ conjugation property, the property of traces, and (4.4),

$$
\begin{aligned}
& \left(\Psi\left(\mathcal{Y}_{a a_{1} ; p}^{a_{1} ;(1)} \otimes \mathcal{Y}_{a_{2} a_{2}^{\prime} ; q}^{a ;(2)}\right)\right)\left(w_{a_{2}} \otimes w_{a_{2}^{\prime}} ; z_{1}, z_{2}+\tau ; \tau\right) \\
& =E\left(\operatorname { T r } _ { W ^ { a _ { 1 } } } \mathcal { Y } _ { a a _ { 1 } ; p } ^ { a _ { 1 } ; ( 1 ) } \left(\mathcal{U}\left(e^{2 \pi i\left(z_{2}+\tau\right)}\right) \cdot\right.\right. \\
& \left.\left.\quad \mathcal{Y}_{a_{2} a_{2}^{\prime} ; q}^{a ;(2)}\left(w_{a_{2}}, z_{1}-\left(z_{2}+\tau\right)\right) w_{a_{2}^{\prime}}, e^{2 \pi i\left(z_{2}+\tau\right)}\right) q_{\tau}^{L(0)-\frac{c}{24}}\right)
\end{aligned}
$$




$$
\begin{aligned}
& =\sum_{a_{3} \in \mathcal{A}} \sum_{i=1}^{N_{a_{2} a_{3}}^{a_{1}}} \sum_{j=1}^{N_{a_{2}^{\prime} a_{1}}^{a_{3}}} F^{-1}\left(\mathcal{Y}_{a_{a 1} ; p}^{a_{1} ;(1)} \otimes \mathcal{Y}_{a_{2} a_{2}^{\prime} ; q}^{a ;(2)} ; \sigma_{23}\left(\mathcal{Y}_{a_{2} a_{1}^{\prime} ; i}^{a_{3}^{\prime} ;(3)}\right) \otimes \sigma_{13}\left(\mathcal{Y}_{a_{3}^{\prime} a_{1} ; j}^{a_{2} ;(4)}\right)\right) . \\
& \cdot E\left(\operatorname{Tr}_{W^{a_{1}}} \sigma_{23}\left(\mathcal{Y}_{a_{2} a_{1}^{\prime} ; i}^{a_{3}^{\prime} ;(3)}\right)\left(\mathcal{U}\left(e^{2 \pi i z_{1}}\right) w_{a_{2}}, e^{2 \pi i z_{1}}\right) .\right. \\
& \left.\cdot \sigma_{13}\left(\mathcal{Y}_{a_{3}^{\prime} a_{1} ; j}^{a_{2} ;(4)}\right)\left(\mathcal{U}\left(e^{2 \pi i\left(z_{2}+\tau\right)}\right) w_{a_{2}^{\prime}}, e^{2 \pi i\left(z_{2}+\tau\right)}\right) q_{\tau}^{L(0)-\frac{c}{24}}\right) \\
& =\sum_{a_{3} \in \mathcal{A}} \sum_{i=1}^{N_{a_{2} a_{3}}^{a_{1}}} \sum_{j=1}^{N_{a_{2}^{\prime} a_{1}}^{a_{3}}} F^{-1}\left(\mathcal{Y}_{a_{a} ; p}^{a_{1} ;(1)} \otimes \mathcal{Y}_{a_{2} a_{2}^{\prime} ; q}^{a ;(2)} ; \sigma_{23}\left(\mathcal{Y}_{a_{2} a_{1}^{\prime} ; i}^{a_{3}^{\prime} ;(3)}\right) \otimes \sigma_{13}\left(\mathcal{Y}_{a_{3}^{\prime} a_{1} ; j}^{a_{2} ;(4)}\right)\right) . \\
& \cdot E\left(\operatorname{Tr}_{W^{a_{1}}} \sigma_{23}\left(\mathcal{Y}_{a_{2} a_{1}^{\prime} ; i}^{a_{3}^{\prime} ;(3)}\right)\left(\mathcal{U}\left(e^{2 \pi i z_{1}}\right) w_{a_{2}}, e^{2 \pi i z_{1}}\right) \cdot\right. \\
& \left.\cdot \sigma_{13}\left(\mathcal{Y}_{a_{3}^{\prime} a_{1} ; j}^{a_{2} ;(4)}\right)\left(\mathcal{U}\left(q_{\tau} e^{2 \pi i z_{2}}\right) w_{a_{2}^{\prime}}, q_{\tau} e^{2 \pi i z_{2}}\right) q_{\tau}^{L(0)-\frac{c}{24}}\right) \\
& =\sum_{a_{3} \in \mathcal{A}} \sum_{i=1}^{N_{a_{2} a_{3}}^{a_{1}}} \sum_{j=1}^{N_{a_{2}^{\prime} a_{1}}^{a_{3}}} F^{-1}\left(\mathcal{Y}_{a a_{1} ; p}^{a_{1} ;(1)} \otimes \mathcal{Y}_{a_{2} a_{2}^{\prime} ; q}^{a ;(2)} ; \sigma_{23}\left(\mathcal{Y}_{a_{2} a_{1}^{\prime} ; i}^{a_{3}^{\prime} ;(3)}\right) \otimes \sigma_{13}\left(\mathcal{Y}_{a_{3}^{\prime} a_{1} ; j}^{a_{2} ;(4)}\right)\right) . \\
& \cdot E\left(\operatorname{Tr}_{W^{a_{1}}} \sigma_{23}\left(\mathcal{Y}_{a_{2} a_{1}^{\prime} ; i}^{a_{3}^{\prime} ;(3)}\right)\left(\mathcal{U}\left(e^{2 \pi i z_{1}}\right) w_{a_{2}}, e^{2 \pi i z_{1}}\right) .\right. \\
& \left.\cdot q_{\tau}^{L(0)-\frac{c}{24}} \sigma_{13}\left(\mathcal{Y}_{a_{3}^{\prime} a_{1} ; j}^{a_{2} ;(4)}\right)\left(\mathcal{U}\left(e^{2 \pi i z_{2}}\right) w_{a_{2}^{\prime}}, e^{2 \pi i z_{2}}\right)\right) \\
& =\sum_{a_{3} \in \mathcal{A}} \sum_{i=1}^{N_{a_{2} a_{3}}^{a_{1}}} \sum_{j=1}^{N_{a_{2}^{\prime} a_{1}}^{a_{3}}} F^{-1}\left(\mathcal{Y}_{a_{a} ; p}^{a_{1} ;(1)} \otimes \mathcal{Y}_{a_{2} a_{2}^{\prime} ; q}^{a ;(2)} ; \sigma_{23}\left(\mathcal{Y}_{a_{2} a_{1}^{\prime} ; i}^{a_{3}^{\prime} ;(3)} \otimes \sigma_{13}\left(\mathcal{Y}_{a_{3}^{\prime} a_{1} ; j}^{a_{2} ;(4)}\right)\right)\right. \\
& \cdot E\left(\operatorname{Tr}_{W^{a_{3}}} \sigma_{13}\left(\mathcal{Y}_{a_{3}^{\prime} a_{1} ; j}^{a_{2} ;(4)}\right)\left(\mathcal{U}\left(e^{2 \pi i z_{2}}\right) w_{a_{2}^{\prime}}, e^{2 \pi i z_{2}}\right) \cdot\right. \\
& \left.\cdot \sigma_{23}\left(\mathcal{Y}_{a_{2} a_{1}^{\prime} ; i}^{a_{3}^{\prime}(3)}\right)\left(\mathcal{U}\left(e^{2 \pi i z_{1}}\right) w_{a_{2}}, e^{2 \pi i z_{1}}\right) q_{\tau}^{L(0)-\frac{c}{24}}\right) .
\end{aligned}
$$

Using (2.22) in [H10, the relations $\sigma_{23}^{2}=1, \sigma_{23} \sigma_{13}=\sigma_{123}$ and the genus-one associativity, we have

$$
\begin{aligned}
& E\left(\operatorname{Tr}_{W^{a_{3}}} \sigma_{13}\left(\mathcal{Y}_{a_{3}^{\prime} a_{1} ; j}^{a_{2} ;(4)}\right)\left(\mathcal{U}\left(e^{2 \pi i z_{2}}\right) w_{a_{2}^{\prime}}, e^{2 \pi i z_{2}}\right) .\right. \\
& \left.\cdot \sigma_{23}\left(\mathcal{Y}_{a_{2} a_{1}^{\prime} ; i}^{a_{3}^{\prime} ;(3)}\right)\left(\mathcal{U}\left(e^{2 \pi i z_{1}}\right) w_{a_{2}}, e^{2 \pi i z_{1}}\right) q_{\tau}^{L(0)-\frac{c}{24}}\right) \\
& =E\left(\operatorname{Tr}_{W^{a_{3}}} \sigma_{23}^{2}\left(\sigma_{13}\left(\mathcal{Y}_{a_{3}^{\prime} a_{1} ; j}^{a_{2} ;(4)}\right)\right)\left(\mathcal{U}\left(e^{2 \pi i z_{2}}\right) w_{a_{2}^{\prime}}, e^{2 \pi i z_{2}}\right) .\right. \\
& \left.\cdot \sigma_{23}\left(\mathcal{Y}_{a_{2} a_{1}^{\prime} ; i}^{\left.a_{3}^{\prime} ; 3\right)}\right)\left(\mathcal{U}\left(e^{2 \pi i z_{1}}\right) w_{a_{2}}, e^{2 \pi i z_{1}}\right) q_{\tau}^{L(0)-\frac{c}{24}}\right) \\
& =e^{-2 \pi i h_{a_{2}}} E\left(\operatorname{Tr}_{W^{a_{3}^{\prime}}} \mathcal{Y}_{a_{2} a_{1}^{\prime} ; i}^{a_{3}^{\prime}(3)}\left(\mathcal{U}\left(e^{2 \pi i z_{1}}\right) e^{\pi i L(0)} w_{a_{2}}, e^{-2 \pi i z_{1}}\right)\right. \\
& \cdot \sigma_{23}\left(\sigma_{13}\left(\mathcal{Y}_{a_{3}^{\prime} a_{1} ; j}^{a_{2} ;(4)}\right)\left(\mathcal{U}\left(e^{2 \pi i z_{2}}\right) e^{\pi i L(0)} w_{a_{2}^{\prime}}, e^{-2 \pi i z_{2}}\right) q_{\tau}^{L(0)-\frac{c}{24}}\right)
\end{aligned}
$$




$$
\begin{aligned}
=e^{-2 \pi i h_{a_{2}}} E\left(\operatorname{Tr}_{W_{3}^{a_{3}^{\prime}}} \mathcal{Y}_{a_{2} a_{1}^{\prime} ; i}^{a_{3}^{\prime}(3)}\left(\mathcal{U}\left(e^{-2 \pi i z_{1}}\right) e^{\pi i L(0)} w_{a_{2}}, e^{-2 \pi i z_{1}}\right)\right. \\
\left.\cdot \sigma_{123}\left(\mathcal{Y}_{a_{3}^{\prime} a_{1} ; j}^{a_{2} ;(4)}\right)\left(\mathcal{U}\left(e^{-2 \pi i z_{2}}\right) e^{\pi i L(0)} w_{a_{2}^{\prime}}, e^{-2 \pi i z_{2}}\right) q_{\tau}^{L(0)-\frac{c}{24}}\right) .
\end{aligned}
$$

We now prove

$$
\begin{aligned}
& E\left(\operatorname{Tr}_{W^{a_{3}^{\prime}}} \mathcal{Y}_{a_{2} a_{1}^{\prime} ; i}^{a_{3}^{\prime}(3)}\left(\mathcal{U}\left(e^{-2 \pi i z_{1}}\right) e^{\pi i L(0)} w_{a_{2}}, e^{-2 \pi i z_{1}}\right) .\right. \\
& \left.\cdot \sigma_{123}\left(\mathcal{Y}_{a_{3}^{\prime} a_{1} ; j}^{a_{2} ;(4)}\right)\left(\mathcal{U}\left(e^{-2 \pi i z_{2}}\right) e^{\pi i L(0)} w_{a_{2}^{\prime}}, e^{-2 \pi i z_{2}}\right) q_{\tau}^{L(0)-\frac{c}{24}}\right)
\end{aligned}
$$

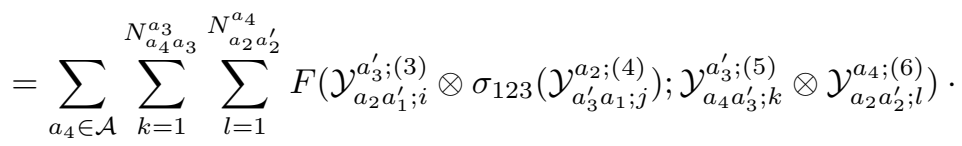

$$
\begin{aligned}
& \cdot E\left(\operatorname { T r } _ { W ^ { a _ { 3 } ^ { \prime } } } \mathcal { Y } _ { a _ { 4 } a _ { 3 } ^ { \prime } ; k } ^ { a _ { 3 } ^ { \prime } ; ( 5 ) } \left(\mathcal{U}\left(e^{-2 \pi i z_{2}}\right) \mathcal{Y}_{a_{2} a_{2}^{\prime} ; l}^{a_{4} ;(6)}\left(e^{\pi i L(0)} w_{a_{2}}, e^{\pi i}\left(z_{1}-z_{2}\right)\right) .\right.\right. \\
& \left.\left.\cdot e^{\pi i L(0)} w_{a_{2}^{\prime}}, e^{-2 \pi i z_{2}}\right) q_{\tau}^{L(0)-\frac{c}{24}}\right) .
\end{aligned}
$$

To prove (4.7), we need only prove that the restrictions of both sides to a subregion of $\widetilde{M}_{1}^{2}$ are equal. So we need only prove that

$$
\begin{aligned}
& \operatorname{Tr}_{W^{a_{3}^{\prime}}} \mathcal{Y}_{a_{2} a_{1}^{\prime} ; i}^{a_{3}^{\prime} ;(3)}\left(\mathcal{U}\left(e^{-2 \pi i z_{1}}\right) e^{\pi i L(0)} w_{a_{2}}, e^{-2 \pi i z_{1}}\right) . \\
& \cdot \sigma_{123}\left(\mathcal{Y}_{a_{3}^{\prime} a_{1} ; j}^{a_{2} ;(4)}\right)\left(\mathcal{U}\left(e^{-2 \pi i z_{2}}\right) e^{\pi i L(0)} w_{a_{2}^{\prime}}, e^{-2 \pi i z_{2}}\right) q_{\tau}^{L(0)-\frac{c}{24}}
\end{aligned}
$$

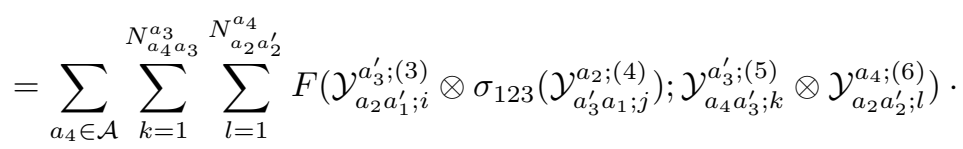

$$
\begin{aligned}
& \cdot \operatorname{Tr}_{W^{a_{3}^{\prime}}} \mathcal{Y}_{a_{4} a_{3}^{\prime} ; k}^{a_{3}^{\prime} ;(5)}\left(\mathcal{U}\left(e^{-2 \pi i z_{2}}\right) \mathcal{Y}_{a_{2} a_{2}^{\prime} ; l}^{\left.a_{4} ; 6\right)}\left(e^{\pi i L(0)} w_{a_{2}}, e^{\pi i}\left(z_{1}-z_{2}\right)\right) .\right. \\
& \left.\cdot e^{\pi i L(0)} w_{a_{2}^{\prime}}, e^{-2 \pi i z_{2}}\right) q_{\tau}^{L(0)-\frac{c}{24}}
\end{aligned}
$$

holds when $\left|q_{\tau}\right|<\left|e^{-2 \pi i z_{2}}\right|<\left|e^{-2 \pi i z_{1}}\right|<1$ and $0<\left|e^{2 \pi i\left(-z_{1}+z_{2}\right)}-1\right|<1$. From the genus-one associativity $((2.9)$ in $[\mathrm{H} 10])$, we see that in this region the left-hand side of (4.8) is equal to

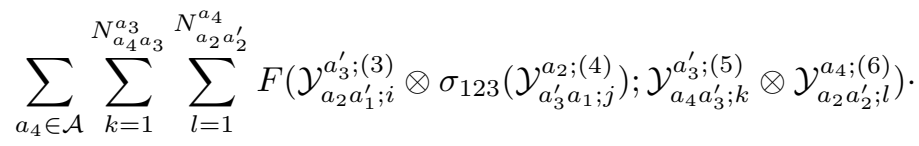

$$
\begin{aligned}
& \cdot \operatorname{Tr}_{W^{a_{3}^{\prime}}} \mathcal{Y}_{a_{4} a_{3}^{\prime} ; k}^{a_{3}^{\prime} ;(5)}\left(\mathcal{U}\left(e^{-2 \pi i z_{2}}\right) \mathcal{Y}_{a_{2} a_{2}^{\prime} ; l}^{a_{4} ;(6)}\left(e^{\pi i L(0)} w_{a_{2}},\left(-z_{1}+z_{2}\right)\right)\right. \\
& \left.\cdot e^{\pi i L(0)} w_{a_{2}^{\prime}}, e^{-2 \pi i z_{2}}\right) q_{\tau}^{L(0)-\frac{c}{24}} .
\end{aligned}
$$

Now in this region, because $\left|e^{-2 \pi i z_{2}}\right|<\left|e^{-2 \pi i z_{1}}\right|$, the imaginary part of $z_{1}$ must be greater than the imaginary part of $z_{2}$. Thus $z_{1}-z_{2}$ is in the upper half-plane. This 
means that $\arg \left(z_{1}-z_{2}\right)<\pi$ and $\arg \left(z_{1}-z_{2}\right)+\pi<2 \pi$. So we have $\arg \left(-\left(z_{1}-z_{2}\right)\right)=$ $\arg \left(z_{1}-z_{2}\right)+\pi$. Now for any $n \in \mathbb{C}$, by our convention,

$$
\begin{aligned}
\left(-z_{1}+z_{2}\right)^{n} & =e^{n \log \left(-z_{1}+z_{2}\right)} \\
& =e^{n \log \left(-\left(z_{1}-z_{2}\right)\right)} \\
& =e^{n\left(\log \left|-\left(z_{1}-z_{2}\right)\right|+i \arg \left(-\left(z_{1}-z_{2}\right)\right)\right)} \\
& =e^{n\left(\log \left|\left(z_{1}-z_{2}\right)\right|+i \arg \left(z_{1}-z_{2}\right)+\pi i\right)} \\
& =e^{n\left(\log \left(z_{1}-z_{2}\right)+\pi i\right)} \\
& =\left(e^{\pi i}\left(z_{1}-z_{2}\right)\right)^{n}
\end{aligned}
$$

This shows that indeed when $\left|q_{\tau}\right|<\left|e^{-2 \pi i z_{2}}\right|<\left|e^{-2 \pi i z_{1}}\right|<1$ and $0<\mid e^{2 \pi i\left(-z_{1}+z_{2}\right)}-$ $1 \mid<1$, (4.9) is equal to the right-hand side of (4.8) and (4.8) holds. Consequently, we obtain (4.7).

Using (2.22) in H10 and the $L(0)$-conjugation formula, we have

$$
\begin{gathered}
E\left(\operatorname { T r } _ { W ^ { a _ { 3 } ^ { \prime } } } \mathcal { Y } _ { a _ { 4 } a _ { 3 } ^ { \prime } ; k } ^ { a _ { 3 } ^ { \prime } ; ( 5 ) } \left(\mathcal{U}\left(e^{-2 \pi i z_{2}}\right) \mathcal{Y}_{a_{2} a_{2}^{\prime} ; l}^{a_{4} ;(6)}\left(e^{\pi i L(0)} w_{a_{2}}, e^{\pi i}\left(z_{1}-z_{2}\right)\right) \cdot\right.\right. \\
\left.\left.\cdot e^{\pi i L(0)} w_{a_{2}^{\prime}}, e^{-2 \pi i z_{2}}\right) q_{\tau}^{L(0)-\frac{c}{24}}\right) \\
=e^{\pi i h_{a_{4}}} E\left(\operatorname { T r } _ { W ^ { a _ { 3 } } } \mathcal { Y } _ { a _ { 4 } a _ { 3 } ; k } ^ { a _ { 3 } ; 5 ) } \left(\mathcal{U}\left(e^{2 \pi i z_{2}}\right) e^{-\pi i L(0)} \cdot\right.\right. \\
\left.\left.\qquad \mathcal{Y}_{a_{2} a_{2}^{\prime} ; l}^{a_{4} ;(6)}\left(e^{\pi i L(0)} w_{a_{2}}, e^{\pi i}\left(z_{1}-z_{2}\right)\right) e^{\pi i L(0)} w_{a_{2}^{\prime}}, e^{2 \pi i z_{2}}\right) q_{\tau}^{L(0)-\frac{c}{24}}\right) \\
=e^{\pi i h_{a_{4}}} E\left(\operatorname{Tr}_{W^{a_{3}}} \mathcal{Y}_{a_{4} a_{3} ; k}^{a_{3} ;(5)}\left(\mathcal{U}\left(e^{2 \pi i z_{2}}\right) \mathcal{Y}_{a_{2} a_{2}^{\prime} ; l}^{a_{4} ;(6)}\left(w_{a_{2}}, z_{1}-z_{2}\right) w_{a_{2}^{\prime}}, e^{2 \pi i z_{2}}\right) q_{\tau}^{L(0)-\frac{c}{24}}\right)
\end{gathered}
$$

Combining (4.5), (4.6), (4.7) and (4.10), we obtain (4.3).

For $a_{1}, a_{2}, a \in \mathcal{A}$, we define

$$
\begin{aligned}
& \alpha\left(\Psi\left(\mathcal{Y}_{a a_{1} ; p}^{a_{1} ;(1)} \otimes \mathcal{Y}_{a_{2} a_{2}^{\prime} ; q}^{a ;(2)}\right)\right): \coprod_{a_{3} \in \mathcal{A}} W^{a_{3}} \otimes W^{a_{3}^{\prime}} \rightarrow \mathbb{G}_{1 ; 2}, \\
& \beta\left(\Psi\left(\mathcal{Y}_{a a_{1} ; p}^{a_{1} ;(1)} \otimes \mathcal{Y}_{a_{2} a_{2}^{\prime} ; q}^{a ;(2)}\right)\right): \coprod_{a_{3} \in \mathcal{A}} W^{a_{3}} \otimes W^{a_{3}^{\prime}} \rightarrow \mathbb{G}_{1 ; 2},
\end{aligned}
$$

by

$$
\begin{aligned}
& \left(\alpha\left(\Psi\left(\mathcal{Y}_{a a_{1} ; p}^{a_{1} ;(1)} \otimes \mathcal{Y}_{a_{2} a_{2}^{\prime} ; q}^{a ;(2)}\right)\right)\right)\left(w_{a_{3}} \otimes w_{a_{3}^{\prime}}\right) \\
& \quad= \begin{cases}\left(\Psi\left(\mathcal{Y}_{a a_{1} ; p}^{a_{1} ;(1)} \otimes \mathcal{Y}_{a_{2} a_{2}^{\prime} ; q}^{a ;(2)}\right)\right)\left(w_{a_{2}} \otimes w_{a_{2}^{\prime}} ; z_{1}, z_{2}-1 ; \tau\right) & a_{3}=a_{2}, \\
0 & a_{3} \neq a_{2},\end{cases} \\
& \left(\beta\left(\Psi\left(\mathcal{Y}_{a a_{1} ; p}^{a_{1} ;(1)} \otimes \mathcal{Y}_{a_{2} a_{2}^{\prime} ; q}^{a ;(2)}\right)\right)\right)\left(w_{a_{3}} \otimes w_{a_{3}^{\prime}}\right) \\
& \quad= \begin{cases}\left(\Psi\left(\mathcal{Y}_{a a_{1} ; p}^{a_{1} ;(1)} \otimes \mathcal{Y}_{a_{2} a_{2}^{\prime} ; q}^{a ;(2)}\right)\right)\left(w_{a_{2}} \otimes w_{a_{2}^{\prime}} ; z_{1}, z_{2}+\tau ; \tau\right) & a_{3}=a_{2} \\
0 & a_{3} \neq a_{2},\end{cases}
\end{aligned}
$$

for $a_{3} \in \mathcal{A}, w_{a_{3}} \in W^{a}$ and $w_{a_{3}^{\prime}} \in W^{a^{\prime}}$. 
Proposition 4.4. For $a_{1}, a_{2} \in \mathcal{A}$, we have

$$
\begin{aligned}
& \alpha\left(\Psi\left(\mathcal{Y}_{a a_{1} ; p}^{a_{1} ;(1)} \otimes \mathcal{Y}_{a_{2} a_{2}^{\prime} ; q}^{a ;(2)}\right)\right) \\
& =e^{-2 \pi i h_{a_{2}}} \sum_{a_{4} \in \mathcal{A}} \sum_{k=1}^{N_{a_{4} a_{1}}^{a_{1}}} \sum_{l=1}^{N_{a_{2} a_{2}^{\prime}}^{a_{4}}}\left(B^{(-1)}\right)^{2} . \\
& \cdot\left(\sigma_{12}\left(\mathcal{Y}_{a a_{1} ; p}^{a_{1} ;(1)}\right) \otimes \sigma_{12}\left(\mathcal{Y}_{a_{2} a_{2}^{\prime} ; q}^{a ;(2)}\right) ; \sigma_{12}\left(\mathcal{Y}_{a_{4} a_{1} ; k}^{a_{1} ;(5)}\right) \otimes \sigma_{12}\left(\mathcal{Y}_{a_{2} a_{2}^{\prime} ; l}^{a_{4} ;(6)}\right)\right) . \\
& \cdot\left(\Psi\left(\mathcal{Y}_{a_{4} a_{1} ; k}^{a_{1} ;(5)} \otimes \mathcal{Y}_{a_{2} a_{2}^{\prime} ; l}^{a_{4} ;(6)}\right)\right)\left(w_{a_{2}} \otimes w_{a_{2}^{\prime}} ; z_{1}, z_{2} ; \tau\right)
\end{aligned}
$$

and

$$
\begin{gathered}
\beta\left(\Psi\left(\mathcal{Y}_{a a_{1} ; p}^{a_{1} ;(1)} \otimes \mathcal{Y}_{a_{2} a_{2}^{\prime} ; q}^{a ;(2)}\right)\right) \\
=e^{-2 \pi i h_{a_{2}}} \sum_{a_{3} \in \mathcal{A}} \sum_{i=1}^{N_{a_{2} a_{3}}^{a_{1} a_{3}}} \sum_{j=1}^{N_{a_{2}^{\prime} a_{1}}^{a_{3}}} \sum_{a_{4} \in \mathcal{A}} \sum_{k=1}^{N_{a_{4} a_{3}}^{a_{3}}} \sum_{l=1}^{N_{a_{2} a_{2}^{\prime}}^{a_{4}}} e^{\pi i h_{a_{4}}} . \\
\cdot F\left(\sigma_{12}\left(\mathcal{Y}_{a a_{1} ; p}^{a_{1} ;(p)}\right) \otimes \sigma_{12}\left(\mathcal{Y}_{a_{2} a_{2}^{\prime} ; j}^{a ;(2)}\right) ; \sigma_{123}\left(\mathcal{Y}_{a_{2} a_{1}^{\prime} ; i}^{a_{3}^{\prime} ;(3)}\right) \otimes \sigma_{132}\left(\mathcal{Y}_{a_{3}^{\prime} a_{1} ; j}^{a_{2} ;(4)}\right)\right) . \\
\cdot F\left(\mathcal{Y}_{a_{2} a_{1}^{\prime} ; i}^{a_{3}^{\prime} ;(3)} \otimes \sigma_{123}\left(\mathcal{Y}_{a_{3}^{\prime} a_{1} ; j}^{a_{2} ;(4)}\right) ; \mathcal{Y}_{a_{4} a_{3}^{\prime} ; k}^{a_{3}^{\prime} ;(5)} \otimes \mathcal{Y}_{a_{2} a_{2}^{\prime} ;(;)}^{a_{4} ;(6)}\right) . \\
\cdot\left(\Psi\left(\mathcal{Y}_{a_{4} ; a_{3} ; k}^{a_{3} ;(5)} \otimes \mathcal{Y}_{a_{2} a_{2}^{\prime} ; l}^{a_{4} ;(6)}\right)\left(w_{a_{2}} \otimes w_{a_{2}^{\prime}} ; z_{1}, z_{2} ; \tau\right) .\right.
\end{gathered}
$$

Proof. Using the definitions of $\alpha$ and

$$
\left(\Psi\left(\mathcal{Y}_{a a_{1} ; p}^{a_{1} ;(1)} \otimes \mathcal{Y}_{a_{2} a_{2}^{\prime} ; q}^{a ;(2)}\right)\right)\left(w_{a_{2}} \otimes w_{a_{2}^{\prime}} ; z_{1}, z_{2} ; \tau\right),
$$

(4.2) and Proposition 3.1 in H10, we have

$$
\begin{aligned}
& \left(\alpha\left(\Psi\left(\mathcal{Y}_{a a_{1} ; p}^{a_{1} ;(1)} \otimes \mathcal{Y}_{a_{2} a_{2}^{\prime} ; q}^{a ;(2)}\right)\right)\right)\left(w_{a_{2}} \otimes w_{a_{2}^{\prime}} ; z_{1}, z_{2} ; \tau\right) \\
& =\left(\Psi\left(\mathcal{Y}_{a a_{1} ; p}^{a_{1} ;(1)} \otimes \mathcal{Y}_{a_{2} a_{2}^{\prime} ; q}^{a ;(2)}\right)\left(w_{a_{2}} \otimes w_{a_{2}^{\prime}} ; z_{1}, z_{2}-1 ; \tau\right)\right. \\
& =\sum_{a \in \mathcal{A}} \sum_{i=1}^{N_{a_{2} a_{3}}^{a_{1}}} \sum_{j=1}^{N_{a_{2}^{\prime} a_{1}}^{a_{3}}} \sum_{a_{4} \in \mathcal{A}} \sum_{k=1}^{N_{a_{4} a_{1}}^{a_{1}}} \sum_{l=1}^{N_{a_{2} a_{2}^{\prime}}^{a_{4}}} e^{-2 \pi i\left(h_{a_{3}}-h_{a_{1}}\right)} . \\
& \cdot F^{-1}\left(\mathcal{Y}_{a a_{1} ; p}^{a_{1} ;(1)} \otimes \mathcal{Y}_{a_{2} a_{2}^{\prime} ; q}^{a ;(2)} ; \mathcal{Y}_{a_{2} a_{3} ; i}^{a_{1} ;(3)} \otimes \mathcal{Y}_{a_{2}^{\prime} a_{1} ; j}^{a_{3}(4)}\right) \cdot \\
& \cdot F\left(\mathcal{Y}_{a_{2} a_{3} ; i}^{a_{1} ;(3)} \otimes \mathcal{Y}_{a_{2}^{\prime} a_{1} ; j}^{a_{3} ;(4)} ; \mathcal{Y}_{a_{4} a_{1} ; k}^{a_{1} ;(5)} \otimes \mathcal{Y}_{a_{2} a_{2}^{\prime} ; l}^{a_{4} ;(6)}\right) \text {. } \\
& \cdot E\left(\operatorname { T r } _ { W ^ { a _ { 1 } } } \mathcal { Y } _ { a _ { 4 } a _ { 1 } ; k } ^ { a _ { 1 } ; ( 5 ) } \left(\mathcal{U}\left(e^{2 \pi i z_{2}}\right) \cdot\right.\right. \\
& \left.\left.\cdot \mathcal{Y}_{a_{2} a_{2}^{\prime} ; l}^{a_{4} ;(6)}\left(w_{a_{2}}, z_{1}-z_{2}\right) w_{a_{2}^{\prime}}, e^{2 \pi i z_{2}}\right) q_{\tau}^{L(0)-\frac{c}{24}}\right) \\
& =e^{-2 \pi i h_{a_{2}}} \sum_{a \in \mathcal{A}} \sum_{i=1}^{N_{a_{2} a_{3}}^{a_{1}}} \sum_{j=1}^{N_{a_{2}^{\prime} a_{1}}^{a_{3}}} \sum_{a_{4} \in \mathcal{A}} \sum_{k=1}^{N_{a_{4} a_{1}}^{a_{1}}} \sum_{l=1}^{N_{a_{2} a_{2}^{\prime}}^{a_{4}}} . \\
& \cdot F\left(\sigma_{12}\left(\mathcal{Y}_{a a_{1} ; p}^{a_{1} ;(1)}\right) \otimes \sigma_{12}\left(\mathcal{Y}_{a_{2} a_{2}^{\prime} ; q}^{a ;(2)}\right) ; \sigma_{12}\left(\mathcal{Y}_{a_{2} a_{3} ; i}^{a_{1} ;(3)}\right) \otimes \sigma_{12}\left(\mathcal{Y}_{a_{2}^{\prime} a_{1} ; j}^{a_{3} ;(4)}\right)\right) . \\
& \cdot e^{-2 \pi i\left(h_{a_{3}}-h_{a_{1}}-h_{a_{2}}\right)} \text {. } \\
& \cdot F^{-1}\left(\sigma_{12}\left(\mathcal{Y}_{a_{2} a_{3} ; i}^{a_{1} ;(3)}\right) \otimes \sigma_{12}\left(\mathcal{Y}_{a_{2}^{\prime} a_{1} ; j}^{a_{3} ;(4)}\right) ; \sigma_{12}\left(\mathcal{Y}_{a_{4} a_{1} ; k}^{a_{1} ;(5)}\right) \otimes \sigma_{12}\left(\mathcal{Y}_{a_{2} a_{2}^{\prime} ; l}^{a_{4} ;(6)}\right)\right) \cdot
\end{aligned}
$$




$$
\begin{gathered}
\cdot E\left(\operatorname { T r } _ { W ^ { a _ { 1 } } } \mathcal { Y } _ { a _ { 4 } a _ { 1 } ; k } ^ { a _ { 1 } ; ( 5 ) } \left(\mathcal{U}\left(e^{2 \pi i z_{2}}\right) \cdot\right.\right. \\
\left.\left.\mathcal{Y}_{a_{2} a_{2}^{\prime} ; l}^{a_{4} ;(6)}\left(w_{a_{2}}, z_{1}-z_{2}\right) w_{a_{2}^{\prime}}, e^{2 \pi i z_{2}}\right) q_{\tau}^{L(0)-\frac{c}{24}}\right) \\
=e^{-2 \pi i h_{a_{2}}} \sum_{a_{4} \in \mathcal{A}} \sum_{k=1}^{N_{a_{4} a_{1}}^{a_{1}}} \sum_{l=1}^{N_{a_{2} a_{2}^{\prime}}^{a_{4}}}\left(B^{(-1)}\right)^{2} \cdot \\
\cdot\left(\sigma_{12}\left(\mathcal{Y}_{a a_{1} ; p}^{a_{1} ;(1)}\right) \otimes \sigma_{12}\left(\mathcal{Y}_{a_{2} a_{2}^{\prime} ; q}^{a ;(2)}\right) ; \sigma_{12}\left(\mathcal{Y}_{a_{4} a_{1} ; k}^{a_{1} ;(5)}\right) \otimes \sigma_{12}\left(\mathcal{Y}_{a_{2} a_{2} ; l}^{a_{4} ;(6)}\right)\right) \\
\cdot\left(\Psi\left(\mathcal{Y}_{a_{4} a_{1} ; k}^{a_{1} ;(5)} \otimes \mathcal{Y}_{a_{2} a_{2}^{\prime} ; l}^{a_{4} ;(6)}\right)\right)\left(w_{a_{2}} \otimes w_{a_{2}^{\prime}} ; z_{1}, z_{2} ; \tau\right),
\end{gathered}
$$

proving (4.13).

Using the definitions of $\beta$,

$$
\Psi\left(\mathcal{Y}_{a a_{1} ; p}^{a_{1} ;(1)} \otimes \mathcal{Y}_{a_{2} a_{2}^{\prime} ; q}^{a ;(2)}\right)\left(w_{a_{2}} \otimes w_{a_{2}^{\prime}} ; z_{1}, z_{2} ; \tau\right),
$$

and (4.3), we have

$$
\begin{aligned}
& \left(\beta\left(\Psi\left(\mathcal{Y}_{a a_{1} ; p}^{a_{1} ;(1)} \otimes \mathcal{Y}_{a_{2} a_{2}^{\prime} ; q}^{a ;(2)}\right)\right)\left(w_{a_{2}} \otimes w_{a_{2}^{\prime}} ; z_{1}, z_{2} ; \tau\right)\right)\left(w_{a_{2}}, w_{a_{2}^{\prime}} ; z_{1}, z_{2} ; \tau\right) \\
& =\Psi\left(\mathcal{Y}_{a a_{1} ; p}^{a_{1} ;(1)} \otimes \mathcal{Y}_{a_{2} a_{2}^{\prime} ; q}^{a ;(2)}\right)\left(w_{a_{2}} \otimes w_{a_{2}^{\prime}} ; z_{1}, z_{2}+\tau ; \tau\right) \\
& =\sum_{a_{3} \in \mathcal{A}} \sum_{i=1}^{N_{a_{2} a_{3}}^{a_{1}}} \sum_{j=1}^{N_{a_{2}^{\prime} a_{1}}^{a_{3}}} \sum_{a_{4} \in \mathcal{A}} \sum_{k=1}^{N_{a_{4} a_{3}}^{a_{3}}} \sum_{l=1}^{N_{a_{2} a_{2}^{\prime}}^{a_{4}}} e^{\pi i\left(-2 h_{a_{2}}+h_{a_{4}}\right)} \text {. } \\
& \cdot F^{-1}\left(\mathcal{Y}_{a a_{1} ; p}^{a_{1} ;(1)} \otimes \mathcal{Y}_{a_{2} a_{2}^{\prime} ; j}^{a ;(2)} ; \sigma_{23}\left(\mathcal{Y}_{a_{2} a_{1}^{\prime} ; i}^{a_{3}^{\prime} ;(3)} \otimes \sigma_{13}\left(\mathcal{Y}_{a_{3}^{\prime} a_{1} ; j}^{a_{2} ;(4)}\right)\right) .\right. \\
& \cdot F\left(\mathcal{Y}_{a_{2} a_{1}^{\prime} ; i}^{a_{3}^{\prime} ;(3)} \otimes \sigma_{123}\left(\mathcal{Y}_{a_{3}^{\prime} a_{1} ; j}^{a_{2} ;(4)}\right) ; \mathcal{Y}_{a_{4} a_{3}^{\prime} ; k}^{a_{3}^{\prime} ;(5)} \otimes \mathcal{Y}_{a_{2} a_{2}^{\prime} ; l}^{a_{4} ;(6)}\right) \\
& \cdot E\left(\operatorname{Tr}_{W^{a_{3}}} \mathcal{Y}_{a_{4} a_{3} ; k}^{a_{3} ;(5)}\left(\mathcal{U}\left(e^{2 \pi i z_{2}}\right) \mathcal{Y}_{a_{2} a_{2}^{\prime} ; l}^{a_{4} ;(6)}\left(w_{a_{2}}, z_{1}-z_{2}\right) w_{a_{2}^{\prime}}, e^{2 \pi i z_{2}}\right) q_{\tau}^{L(0)-\frac{c}{24}}\right) \\
& =e^{-2 \pi i h_{a_{2}}} \sum_{a_{3} \in \mathcal{A}} \sum_{i=1}^{N_{a_{2} a_{3}}^{a_{1}}} \sum_{j=1}^{N_{a_{2}^{\prime} a_{1}}^{a_{3}}} \sum_{a_{4} \in \mathcal{A}} \sum_{k=1}^{N_{a_{4} a_{3}}^{a_{3}}} \sum_{l=1}^{N_{a_{2} a_{2}}^{a_{4}}} e^{\pi i h_{a_{4}}} . \\
& \cdot F\left(\sigma_{12}\left(\mathcal{Y}_{a a_{1} ; p}^{a_{1} ;(1)}\right) \otimes \sigma_{12}\left(\mathcal{Y}_{a_{2} a_{2}^{\prime} ; j}^{a ;(2)}\right) ; \sigma_{123}\left(\mathcal{Y}_{a_{2} a_{1}^{\prime} ; i}^{a_{3}^{\prime} ;(3)}\right) \otimes \sigma_{132}\left(\mathcal{Y}_{a_{3}^{\prime} a_{1} ; j}^{a_{2} ;(4)}\right)\right) \text {. } \\
& \cdot F\left(\mathcal{Y}_{a_{2} a_{1}^{\prime} ; i}^{a_{3}^{\prime}(3)} \otimes \sigma_{123}\left(\mathcal{Y}_{a_{3}^{\prime} a_{1} ; j}^{a_{2} ;(4)}\right) ; \mathcal{Y}_{a_{4} a_{3}^{\prime} ; k}^{a_{3}^{\prime} ;(5)} \otimes \mathcal{Y}_{a_{2} a_{2}^{\prime} ; l}^{a_{4} ;(6)}\right) \text {. } \\
& \cdot\left(\Psi\left(\mathcal{Y}_{a_{4} a_{3} ; k}^{\left.a_{3} ; 5\right)} \otimes \mathcal{Y}_{a_{2} a_{2}^{\prime} ; l}^{a_{4} ;(6)}\right)\right)\left(w_{a_{2}} \otimes w_{a_{2}^{\prime}} ; z_{1}, z_{2} ; \tau\right),
\end{aligned}
$$

proving (4.14).

By Proposition 2.2 in [H10, for any bases $\left\{\mathcal{Y}_{a a_{1} ; p}^{a_{1} ;(1)} \mid p=1, \ldots, N_{a a_{1}}^{a_{1}}\right\}$ and $\left.\left\{\mathcal{Y}_{a_{2} a_{2}^{\prime} ; q}^{a ;(2)}\right) \mid q=1, \ldots, N_{a_{2} a_{2}^{\prime}}^{a}\right\}$,

$$
\left(\Psi\left(\mathcal{Y}_{a a_{1} ; p}^{a_{1} ;(1)} \otimes \mathcal{Y}_{a_{2} a_{2}^{\prime} ; q}^{a ;(2)}\right)\right)\left(w_{a_{2}} \otimes w_{a_{2}^{\prime}} ; z_{1}, z_{2} ; \tau\right)
$$

for $a_{1}, a_{2}, a \in \mathcal{A}, p=1, \ldots, N_{a a_{1}}^{a_{1}}$ and $q=1, \ldots, N_{a_{2} a_{2}^{\prime}}^{a}$ form a basis of $\mathcal{F}_{1 ; 2}$. We use

$$
\alpha\left(\Psi\left(\mathcal{Y}_{a a_{1} ; p}^{a_{1} ;(1)} \otimes \mathcal{Y}_{a_{2} a_{2}^{\prime} ; q}^{a ;(2)}\right) ; \Psi\left(\mathcal{Y}_{a_{4} a_{3} ; k}^{a_{3} ;(5)} \otimes \mathcal{Y}_{a_{2} a_{2}^{\prime} ; l}^{a_{4} ;(6)}\right)\right)
$$


and

$$
\beta\left(\Psi\left(\mathcal{Y}_{a a_{1} ; p}^{a_{1} ;(1)} \otimes \mathcal{Y}_{a_{2} a_{2}^{\prime} ; q}^{a ;(2)}\right) ; \Psi\left(\mathcal{Y}_{a_{4} a_{3} ; k}^{a_{3} ;(5)} \otimes \mathcal{Y}_{a_{2} a_{2} ; l}^{a_{4} ;(6)}\right)\right)
$$

to denote the matrix elements of $\alpha$ and $\beta$, respectively, under the bases

$$
\Psi\left(\mathcal{Y}_{a a_{1} ; p}^{a_{1} ;(1)} \otimes \mathcal{Y}_{a_{2} a_{2}^{\prime} ; q}^{a ;(2)}\right)
$$

and

$$
\Psi\left(\mathcal{Y}_{a_{4} a_{3} ; k}^{a_{3} ;(5)} \otimes \mathcal{Y}_{a_{2} a_{2}^{\prime} ; l}^{a_{4} ;(6)}\right) .
$$

Corollary 4.5. The matrix elements of $\alpha$ and $\beta$ are given by

$$
\begin{aligned}
& \alpha\left(\Psi\left(\mathcal{Y}_{a a_{1} ; p}^{a_{1} ;(1)} \otimes \mathcal{Y}_{a_{2} a_{2}^{\prime} ; q}^{a ;(2)}\right) ; \Psi\left(\mathcal{Y}_{a_{4} a_{3} ; k}^{a_{3} ;(5)} \otimes \mathcal{Y}_{a_{2} a_{2}^{\prime} ; l}^{a_{4} ;(6)}\right)\right) \\
& =\delta_{a_{3} a_{1}} e^{-2 \pi i h_{a_{2}}} \\
& \quad \cdot\left(B^{(-1)}\right)^{2}\left(\sigma_{12}\left(\mathcal{Y}_{a a_{1} ; p}^{a_{1} ;(1)}\right) \otimes \sigma_{12}\left(\mathcal{Y}_{a_{2} a_{2}^{\prime} ; q}^{a ;(2)}\right) ; \sigma_{12}\left(\mathcal{Y}_{a_{4} a_{1} ; k}^{a_{1} ;(5)}\right) \otimes \sigma_{12}\left(\mathcal{Y}_{a_{2} a_{2}^{\prime} ; l}^{a_{4} ;(6)}\right)\right)
\end{aligned}
$$

and

$$
\begin{aligned}
& \beta\left(\Psi\left(\mathcal{Y}_{a a_{1} ; p}^{a_{1} ;(1)} \otimes \mathcal{Y}_{a_{2} a_{2}^{\prime} ; q}^{a ;(2)}\right) ; \Psi\left(\mathcal{Y}_{a_{4} a_{3} ; k}^{a_{3} ;(5)} \otimes \mathcal{Y}_{a_{2} a_{2}^{\prime} ; l}^{a_{4} ;(6)}\right)\right) \\
& =e^{-2 \pi i h_{a_{2}}} \sum_{i=1}^{N_{a_{2} a_{3}}^{a_{1}}} \sum_{j=1}^{N_{a_{2}^{\prime} a_{1}}^{a_{3}}} e^{\pi i h_{a_{4}}} . \\
& \cdot F\left(\sigma_{12}\left(\mathcal{Y}_{a a_{1} ; p}^{a_{1} ;(1)}\right) \otimes \sigma_{12}\left(\mathcal{Y}_{a_{2} a_{2}^{\prime} ; q}^{a ;(2)}\right) ; \sigma_{123}\left(\mathcal{Y}_{a_{2} a_{1}^{\prime} ; i}^{a_{3}^{\prime} ;(3)}\right) \otimes \sigma_{132}\left(\mathcal{Y}_{a_{3}^{\prime} a_{1} ; j}^{a_{2} ;(4)}\right)\right) . \\
& \cdot F\left(\mathcal{Y}_{a_{2} a_{1}^{\prime} ; i}^{a_{3}^{\prime} ;(3)} \otimes \sigma_{123}\left(\mathcal{Y}_{a_{3}^{\prime} a_{1} ; j}^{a_{2} ;(4)}\right) ; \mathcal{Y}_{a_{4} a_{3}^{\prime} ; k}^{a_{3}^{\prime} ;(5)} \otimes \mathcal{Y}_{a_{2} a_{2}^{\prime} ; l}^{a_{1} ;(6)}\right) .
\end{aligned}
$$

Proof. This corollary follows directly from the definitions of the matrix elements and the formulas (4.13) and (4.14).

For $a \in \mathcal{A}$, we define an action of a modular transformation $S$ on $\mathcal{F}_{1 ; 1}$ as follows: for $a_{1} \in \mathcal{A}, k=1, \ldots, N_{a a_{1}}^{a_{1}}$ and $w_{a} \in W^{a}$, let

$$
\begin{aligned}
S\left(\Psi(\mathcal{Y})\left(w_{a} ; \tau\right)\right) & =\Psi(\mathcal{Y})\left(\left(-\frac{1}{\tau}\right)^{L(0)} w_{a} ;-\frac{1}{\tau}\right) \\
& =\operatorname{Tr}_{W^{a_{1}}} \mathcal{Y}\left(\mathcal{U}\left(e^{-2 \pi i \frac{z}{\tau}}\right)\left(-\frac{1}{\tau}\right)^{L(0)} w_{a}, e^{-2 \pi i \frac{z}{\tau}}\right) q_{-\frac{1}{\tau}}^{L(0)-\frac{c}{24}}
\end{aligned}
$$

Here we have used our convention

$$
\left(-\frac{1}{\tau}\right)^{L(0)}=e^{\left(\log \left(-\frac{1}{\tau}\right)\right) L(0)}
$$


Note that by the modular invariance of genus-one one-point functions proved in [H7] (see Theorem 2.4), $S\left(\Psi(\mathcal{Y})(u ; \tau)\right.$ ) is indeed in $\mathcal{F}_{1 ; 1}$. Thus we do obtain maps $S: \mathcal{F}_{1 ; 1} \rightarrow \mathcal{F}_{1 ; 1}$.

For $a \in \mathcal{A}$, this action of $S$ on $\mathcal{F}_{1 ; 1}$ can be extended to an action of $S$ on $\mathcal{F}_{1 ; 2}^{a}$. For intertwining operators $\mathcal{Y}_{1}$ and $\mathcal{Y}_{2}$ of types for intertwining operators of types $\left(\begin{array}{c}W^{a_{1}} \\ W^{a} W^{a_{1}}\end{array}\right)$ and $\left(\begin{array}{c}W^{a} \\ W^{a_{2}} W^{a_{2}^{\prime}}\end{array}\right)$, respectively, we define

$$
\left(S\left(\Psi\left(\mathcal{Y}_{1} \otimes \mathcal{Y}_{2}\right)\right)\right)\left(w_{a}, w_{a^{\prime}} ; z_{1}, z_{2} ; \tau\right)=0
$$

when $a \neq a_{2}$ and

$$
\begin{aligned}
&((S)\left.\left.\Psi\left(\mathcal{Y}_{1} \otimes \mathcal{Y}_{2}\right)\right)\right)\left(w_{a_{2}}, w_{a_{2}^{\prime}} ; z_{1}, z_{2} ; \tau\right) \\
&= E\left(\operatorname { T r } _ { W ^ { a _ { 1 } } } \mathcal { Y } _ { 1 } \left(\mathcal{U}\left(e^{-2 \pi i \frac{z_{2}}{\tau}}\right)\left(-\frac{1}{\tau}\right)^{L(0)} \cdot\right.\right. \\
&\left.\left.\cdot \mathcal{Y}_{2}\left(w_{a_{2}}, z_{1}-z_{2}\right) w_{a_{2}^{\prime}}, e^{-2 \pi i \frac{z_{2}}{\tau}}\right) q_{-\frac{1}{\tau}}^{L(0)-\frac{c}{24}}\right) \\
&=E\left(\operatorname { T r } _ { W ^ { a _ { 1 } } } \mathcal { Y } _ { 1 } \left(\mathcal{U}\left(e^{-2 \pi i \frac{z_{2}}{\tau}}\right) \cdot\right.\right. \\
&\left.\quad \mathcal{Y}_{2}\left(\left(-\frac{1}{\tau}\right)^{L(0)} w_{a_{2}},-\frac{1}{\tau} z_{1}-\left(-\frac{1}{\tau} z_{2}\right)\right)\left(-\frac{1}{\tau}\right)^{L(0)} w_{a_{2}^{\prime}}, e^{-2 \pi i \frac{z_{2}}{\tau}}\right) . \\
&\left.\quad q_{-\frac{1}{\tau}}^{L(0)-\frac{c}{24}}\right) \\
&=\Psi\left(\mathcal{Y}_{1} \otimes \mathcal{Y}_{2}\right)\left(\left(-\frac{1}{\tau}\right)^{L(0)} w_{a},\left(-\frac{1}{\tau}\right)^{L(0)} w_{a^{\prime}} ;-\frac{1}{\tau} z_{1},-\frac{1}{\tau} z_{2} ;-\frac{1}{\tau}\right) .
\end{aligned}
$$

Proposition 4.6. We have the following formula:

$$
S \alpha=\beta S \text {. }
$$

Proof. We have

$$
\begin{aligned}
& \left(\beta\left(S\left(\Psi\left(\mathcal{Y}_{a a_{1} ; p}^{a_{1} ;(1)} \otimes \mathcal{Y}_{a_{2} a_{2}^{\prime} ; q}^{a ;(2)}\right)\right)\right)\right)\left(w_{a_{2}} \otimes w_{a_{2}^{\prime}} ; z_{1}, z_{2} ; \tau\right) \\
& =\left(S\left(\Psi\left(\mathcal{Y}_{a a_{1} ; p}^{a_{1} ;(1)} \otimes \mathcal{Y}_{a_{2} a_{2}^{\prime} ; q}^{a ;(2)}\right)\right)\left(w_{a_{2}} \otimes w_{a_{2}^{\prime}} ; z_{1}, z_{2}+\tau ; \tau\right)\right. \\
& =E\left(\operatorname { T r } _ { W ^ { a _ { 1 } } } \mathcal { Y } _ { a a _ { 1 } ; p } ^ { a _ { 1 } } \left(\mathcal{U}\left(e^{-2 \pi i \frac{z_{2}+\tau}{\tau}}\right)\left(-\frac{1}{\tau}\right)^{L(0)}\right.\right. \text {. } \\
& \left.\left.\mathcal{Y}_{a_{2} a_{2}^{\prime} ; q}^{a}\left(w_{a_{2}}, z_{1}-\left(z_{2}+\tau\right)\right) w_{a_{2}^{\prime}}, e^{-2 \pi i \frac{z_{2}+\tau}{\tau}}\right) q_{-\frac{1}{\tau}}^{L(0)-\frac{c}{24}}\right) \\
& =E\left(\operatorname { T r } _ { W ^ { a _ { 1 } } } \mathcal { Y } _ { a a _ { 1 } ; p } ^ { a _ { 1 } } \left(\mathcal{U}\left(e^{2 \pi i\left(-\frac{z_{2}}{\tau}-1\right)}\right) .\right.\right. \\
& \mathcal{Y}_{a_{2} a_{2}^{\prime} ; q}^{a}\left(\left(-\frac{1}{\tau}\right)^{L(0)} w_{a_{2}},-\frac{1}{\tau} z_{1}-\left(-\frac{1}{\tau} z_{2}-1\right)\right) . \\
& \left.\left.\cdot\left(-\frac{1}{\tau}\right)^{L(0)} w_{a_{2}^{\prime}}, e^{2 \pi i\left(-\frac{z_{2}}{\tau}-1\right)}\right) q_{-\frac{1}{\tau}}^{L(0)-\frac{c}{24}}\right)
\end{aligned}
$$




$$
\begin{array}{r}
=\left(\Psi\left(\mathcal{Y}_{a a_{1} ; p}^{\left.\left.a_{1 ;(1)} \otimes \mathcal{Y}_{a_{2} a_{2}^{\prime} ; q}^{a ;(2)}\right)\right)\left(\left(-\frac{1}{\tau}\right)^{L(0)} w_{a_{2}}\right) \otimes(}\left(-\frac{1}{\tau}\right)^{L(0)} w_{a_{2}^{\prime}}\right) ;\right. \\
\left.-\frac{1}{\tau} z_{1},-\frac{1}{\tau} z_{2}-1 ;-\frac{1}{\tau}\right) \\
=\left(\alpha ( \Psi ( \mathcal { Y } _ { a a _ { 1 } ; p } ^ { a _ { 1 } ; ( 1 ) } \otimes \mathcal { Y } _ { a _ { 2 } a _ { 2 } ^ { \prime } ; q } ^ { a ; ( 2 ) } ) ) \left(\left(\left(-\frac{1}{\tau}\right)^{L(0)} w_{a_{2}}\right) \otimes\left(\left(-\frac{1}{\tau}\right)^{L(0)} w_{a_{2}^{\prime}}\right) ;\right.\right. \\
=\left(S\left(\alpha\left(\Psi_{a_{1}, a_{2}, e}^{1,1}\right)\right)\right)\left(w_{a_{2}}, w_{a_{2}^{\prime}} ; z_{1}, z_{2} ; \tau\right) .
\end{array}
$$

Thus we obtain (4.17).

For fixed $a \in \mathcal{A}$, we know that $\Psi\left(\mathcal{Y}_{a a_{1} ; p}^{a_{1} ;(1)}\right)$ for $a_{1} \in \mathcal{A}$ form a basis of $\mathcal{F}_{1 ; 1}^{a}$, and there exist unique $S\left(\mathcal{Y}_{a a_{1} ; p}^{a_{1} ;(1)} ; \mathcal{Y}_{a a_{2} ; k}^{a_{2} ;(2)}\right)$ in $\mathbb{C}$ for $a_{1}, a_{2} \in \mathcal{A}, p=1, \ldots, N_{a a_{1}}^{a_{1}}$ and $k=1, \ldots, N_{a a_{2}}^{a_{2}}$ such that

$$
S\left(\Psi\left(\mathcal{Y}_{a a_{1} ; p}^{a_{1} ;(1)}\right)\right)=\sum_{a_{2} \in \mathcal{A}} \sum_{k=1}^{N_{a a_{2}}^{a_{2}}} S\left(\mathcal{Y}_{a a_{1} ; p}^{a_{1} ;(1)} ; \mathcal{Y}_{a a_{2} ; k}^{a_{2} ;(2)}\right) \Psi\left(\mathcal{Y}_{a a_{2} ; k}^{a_{2} ;(2)}\right)
$$

Clearly we also have

$$
S\left(\Psi\left(\mathcal{Y}_{a a_{1} ; p}^{a_{1} ;(1)} \otimes \mathcal{Y}_{a_{2} a_{2}^{\prime} ; q}^{a ;(2)}\right)\right)=\sum_{a_{3} \in \mathcal{A}} \sum_{k=1}^{N_{a a_{3}}^{a_{3}}} S\left(\mathcal{Y}_{a a_{1} ; p}^{a_{1} ;(1)} ; \mathcal{Y}_{a a_{3} ; k}^{a_{3} ;(3)}\right) \Psi\left(\mathcal{Y}_{a a_{3} ; k}^{a_{3} ;(3)} \otimes \mathcal{Y}_{a_{2} a_{2}^{\prime} ; q}^{a ;(2)}\right)
$$

for $a_{1}, a_{2}, a_{3} \in \mathcal{A}$.

The following theorem is a generalization of Theorem 4.6 in [H10]:

Theorem 4.7. For $a_{1}, a_{2}, a_{3} \in \mathcal{A}$, we have

$$
\begin{aligned}
& \sum_{k=1}^{N_{a_{4} a_{1}}^{a_{1}}} S\left(\mathcal{Y}_{a_{4} a_{1} ; k}^{a_{1} ;(3)} ; \mathcal{Y}_{a_{4} a_{5} ; m}^{a_{5} ;(5)}\right) . \\
& \cdot\left(B^{(-1)}\right)^{2}\left(\sigma_{12}\left(\mathcal{Y}_{a_{3} a_{1} ; p}^{a_{1} ;(1)}\right) \otimes \sigma_{12}\left(\mathcal{Y}_{a_{2} a_{2}^{\prime} ; q}^{a_{3} ;(2)}\right) ; \sigma_{12}\left(\mathcal{Y}_{a_{4} a_{1} ; k}^{a_{1} ;(3)}\right) \otimes \sigma_{12}\left(\mathcal{Y}_{a_{2} a_{2}^{\prime} ; l}^{a_{4} ;(4)}\right)\right) \\
& =\sum_{a_{6} \in \mathcal{A}} \sum_{r=1}^{N_{a_{3} a_{6}}^{a_{6}}} \sum_{i=1}^{N_{a_{2} a_{5}}^{a_{6}}} \sum_{j=1}^{N_{a_{2}^{\prime} a_{6}}^{a_{5}}} e^{\pi i h_{a_{4}}} S\left(\mathcal{Y}_{a_{3} a_{1} ; p}^{a_{1} ;(1)} ; \mathcal{Y}_{a_{3} a_{6} ; r}^{a_{6} ;(6)}\right) \text {. } \\
& \cdot F\left(\sigma_{12}\left(\mathcal{Y}_{a_{3} a_{6} ; r}^{a_{6} ;(6)}\right) \otimes \sigma_{12}\left(\mathcal{Y}_{a_{2} a_{2}^{\prime} ; q}^{a_{3} ;(2)}\right) ; \sigma_{123}\left(\mathcal{Y}_{a_{2} a_{6}^{\prime} ; i}^{a_{5}^{\prime} ;(7)}\right) \otimes \sigma_{132}\left(\mathcal{Y}_{a_{5}^{\prime} a_{6} ; j}^{a_{2} ;(8)}\right)\right) . \\
& \cdot F\left(\mathcal{Y}_{a_{2} a_{6}^{\prime} ; i}^{a_{5}^{\prime} ;(7)} \otimes \sigma_{123}\left(\mathcal{Y}_{a_{5}^{\prime} a_{6} ; j}^{a_{2} ;(8)}\right) ; \mathcal{Y}_{a_{4} a_{5}^{\prime} ; k}^{a_{5}^{\prime} ;(5)} \otimes \mathcal{Y}_{a_{2} a_{2}^{\prime} ; l}^{a_{4} ;(4)}\right) \text {. }
\end{aligned}
$$

Proof. This follows from (4.17), (4.15) and (4.16) immediately. 
Corollary 4.8. For $a_{1}, a_{2}, a_{3} \in \mathcal{A}$, we have

$$
\begin{aligned}
& S_{e}^{a_{1}}\left(B^{(-1)}\right)^{2}\left(\sigma_{12}\left(\mathcal{Y}_{a_{3} a_{1} ; p}^{a_{1} ;(1)}\right) \otimes \sigma_{12}\left(\mathcal{Y}_{a_{2} a_{2}^{\prime} ; q}^{a_{3} ;(2)}\right) ; \mathcal{Y}_{a_{1} e ; 1}^{a_{1}} \otimes \mathcal{Y}_{a_{2}^{\prime} a_{2} ; l}^{e}\right) \\
& =\sum_{r=1}^{N_{a_{3} a_{2}}^{a_{2}}} S\left(\mathcal{Y}_{a_{3} a_{1} ; p}^{a_{1} ;(1)} ; \mathcal{Y}_{a_{3} a_{2} ; r}^{a_{2} ;(6)}\right) . \\
& \quad \cdot F\left(\sigma_{12}\left(\mathcal{Y}_{a_{3} a_{2} ; r}^{a_{2} ;(6)}\right) \otimes \sigma_{12}\left(\mathcal{Y}_{a_{2} a_{2} ; ;}^{a_{3} ;(2)}\right) ; \mathcal{Y}_{e a_{2} ; 1}^{a_{2}} \otimes \mathcal{Y}_{a_{2} a_{2}^{\prime} ; 1}^{e}\right) \\
& \quad \cdot F\left(\mathcal{Y}_{a_{2} a_{2}^{\prime} ; 1}^{e} \otimes \mathcal{Y}_{a_{2}^{\prime} e ; 1}^{a_{2}^{\prime}} ; \mathcal{Y}_{e e ; 1}^{e} \otimes \mathcal{Y}_{a_{2} a_{2}^{\prime} ; 1}^{e}\right) .
\end{aligned}
$$

Proof. This follows immediately from (4.19) by taking $a_{4}=a_{5}=e$.

Lemma 4.9. For any $a \in \mathcal{A}$,

$$
F\left(\mathcal{Y}_{a a^{\prime} ; 1}^{e} \otimes \mathcal{Y}_{a^{\prime} e ; 1}^{a^{\prime}} ; \mathcal{Y}_{e e ; 1}^{e} \otimes \mathcal{Y}_{a a^{\prime} ; 1}^{e}\right)=1
$$

Proof. Note that $\mathcal{Y}_{e e ; 1}^{e}=Y$ is the vertex operator map for the vertex operator algebra and $\mathcal{Y}_{e a ; 1}^{a}=Y_{W^{a}}$ is the vertex operator map for the $V$-module $W^{a}$. For $u, v \in V, w_{a} \in W^{a}$ and $w_{a^{\prime}} \in W^{a^{\prime}}$, we have

$$
\begin{aligned}
& \left(u, \mathcal{Y}_{e e ; 1}^{e}\left(\mathcal{Y}_{a a^{\prime} ; 1}^{e}\left(w_{a}, z_{1}-z_{2}\right) w_{a^{\prime}}, z_{2}\right) v\right) \\
& \quad=\left(u, Y\left(\mathcal{Y}_{a a^{\prime} ; 1}^{e}\left(w_{a}, z_{1}-z_{2}\right) w_{a^{\prime}}, z_{2}\right) v\right) \\
& \quad=\left(e^{z_{2} L(1)} u, Y\left(v,-z_{2}\right) \mathcal{Y}_{a a^{\prime} ; 1}^{e}\left(w_{a}, z_{1}-z_{2}\right) w_{a^{\prime}}\right)
\end{aligned}
$$

in the region $\left|z_{2}\right|>\left|z_{1}-z_{2}\right|>0$. Since $\mathcal{Y}_{a a^{\prime} ; 1}^{e}$ is an intertwining operator, we know that the product of $Y$ and $\mathcal{Y}_{a a^{\prime} ; 1}^{e}$ satisfy commutativity. Thus the analytic extension of the right-hand side of (4.21) to the region $\left|z_{1}-z_{2}\right|>\left|z_{2}\right|>0$ is equal to

$$
\begin{aligned}
& \left(e^{z_{2} L(1)} u, \mathcal{Y}_{a a^{\prime} ; 1}^{e}\left(w_{a}, z_{1}-z_{2}\right) Y_{W^{a^{\prime}}}\left(v,-z_{2}\right) w_{a^{\prime}}\right) \\
& \quad=\left(u, e^{z_{2} L(-1)} \mathcal{Y}_{a a^{\prime} ; 1}^{e}\left(w_{a}, z_{1}-z_{2}\right) Y_{W^{a^{\prime}}}\left(v,-z_{2}\right) w_{a^{\prime}}\right) .
\end{aligned}
$$

Using the $L(-1)$-conjugation formula for intertwining operators and the definition of the intertwining operator $\mathcal{Y}_{a^{\prime} e ; 1}^{a^{\prime}}$, we see that in the region given by $\left|z_{1}-z_{2}\right|>$ $\left|z_{2}\right|>0$ and $\left|z_{1}\right|>\left|z_{2}\right|>0$, the right-hand side of (4.22) is equal to

$$
\begin{gathered}
\left(u, \mathcal{Y}_{a a^{\prime} ; 1}^{e}\left(w_{a}, z_{1}\right) e^{z_{2} L(-1)} Y_{W^{a^{\prime}}}\left(v,-z_{2}\right) w_{a^{\prime}}\right) \\
=\left(u, \mathcal{Y}_{a a^{\prime} ; 1}^{e}\left(w_{a}, z_{1}\right) \mathcal{Y}_{a^{\prime} e ; 1}^{a^{\prime}}\left(w_{a^{\prime}}, z_{2}\right) v\right) .
\end{gathered}
$$

Thus we see that the left-hand side of (4.21) can be analytically extended to the right-hand side of (4.23). Since the left-hand side of (4.21) and the right-hand side of (4.23) are well defined in the regions $\left|z_{2}\right|>\left|z_{1}-z_{2}\right|>0$ and $\left|z_{1}\right|>\left|z_{2}\right|>0$, respectively, they are equal in the intersection $\left|z_{1}\right|>\left|z_{2}\right|>\left|z_{1}-z_{2}\right|>0$. By the definition of the fusing matrix element $F\left(\mathcal{Y}_{a a^{\prime} ; 1}^{e} \otimes \mathcal{Y}_{a^{\prime} e ; 1}^{a^{\prime}} ; \mathcal{Y}_{e e ; 1}^{e} \otimes \mathcal{Y}_{a a^{\prime} ; 1}^{e}\right)$, we see that it is equal to 1 .

We also have:

Lemma 4.10. For any $a_{2}, a_{3} \in \mathcal{A}$, we have

$F\left(\sigma_{12}\left(\mathcal{Y}_{a_{3} a_{2} ; r}^{a_{2} ;(6)}\right) \otimes \sigma_{12}\left(\mathcal{Y}_{a_{2} a_{2}^{\prime} ; q}^{a_{3} ;(2)}\right) ; \mathcal{Y}_{e a_{2} ; 1}^{a_{2}} \otimes \mathcal{Y}_{a_{2} a_{2}^{\prime} ; 1}^{e}\right)=\left\langle\sigma_{132}\left(\mathcal{Y}_{a_{3} a_{2} ; r}^{a_{2} ;(6)}\right), \sigma_{12}\left(\mathcal{Y}_{a_{2} a_{2}^{\prime} ; q^{\prime}}^{a_{3} ;(2)}\right)_{\mathcal{V}_{a_{2} a_{2}^{\prime}}^{a_{3}^{\prime}}}\right.$.

Proof. This follows directly from (3.3) in [HK]. 
For $a_{1}, a_{2}, a_{3} \in \mathcal{A}$, let

$$
(\cdot, \cdot)_{\mathcal{V}_{a_{1} a_{2}}^{a_{3}}}=\frac{\sqrt{F_{a_{3}}}}{\sqrt{F_{a_{1}}} \sqrt{F_{a_{2}}}}\langle\cdot, \cdot\rangle_{\mathcal{V}_{a_{1} a_{2}}^{a_{3}}},
$$

where $\langle\cdot, \cdot\rangle_{\mathcal{V}_{a_{1} a_{2}}^{a_{3}}}$ is the bilinear form constructed in [HK]. Then Proposition 3.7 in [HK] actually says that $(\cdot, \cdot)_{\mathcal{V}_{a_{1} a_{2}}^{a_{3}}}$ is invariant under the action of $S_{3}$.

Now for $a_{2}, a_{3} \in \mathcal{A}$, we choose

$$
\mathcal{Y}_{a_{2} a_{2}^{\prime} ; i}^{a_{3} ;(2)}=\sigma_{13}\left(\mathcal{Y}_{a_{3}^{\prime} a_{2}^{\prime} ; i}^{\prime ; a_{2}^{\prime} ;(6)}\right)
$$

for $i=1, \ldots, N_{a_{3}^{\prime} a_{2}^{\prime}}^{a^{\prime}}$, where $\left\{\mathcal{Y}_{a_{1}^{\prime} a_{2}^{\prime} ; i}^{\prime} \mid i=1, \ldots, N_{a_{1}^{\prime} a_{2}^{\prime}}^{a_{3}^{\prime}}\right\}$ is the dual basis of $\left\{\mathcal{Y}_{a_{1} a_{2} ; i}^{a_{3}} \mid\right.$ $\left.i=1, \ldots, N_{a_{1} a_{2}}^{a_{3}}\right\}$ with respect to the bilinear form $\langle\cdot, \cdot\rangle_{\mathcal{V}_{a_{1} a_{2}}^{a_{3}} \text {. }}$.

The following result generalizes (5.15) in [H10, which, as was shown in H11, is equivalent to the nondegeneracy of the semisimple ribbon (tensor) category of modules for $V$ :

Theorem 4.11. For $a_{1}, a_{2}, a_{3} \in \mathcal{A}$, we have

$$
\begin{aligned}
& S\left(\mathcal{Y}_{a_{3} a_{1} ; p}^{a_{1} ;(1)} ; \mathcal{Y}_{a_{3} ; a_{2} ; r}^{a_{2} ;(6)}\right) \\
& \quad=\frac{F_{a_{3}} S_{e}^{e}}{F_{a_{1}} F_{a_{2}}}\left(B^{(-1)}\right)^{2}\left(\sigma_{12}\left(\mathcal{Y}_{a_{3} a_{1} ; p}^{a_{1} ;(1)}\right) \otimes \sigma_{132}\left(\mathcal{Y}_{a_{3}^{\prime} a_{2}^{\prime} ; r}^{\prime ;} ;(6) ; \mathcal{Y}_{a_{1} ; 1}^{a_{1}} \otimes \mathcal{Y}_{a_{2}^{\prime} a_{2} ; 1}^{e}\right) .\right.
\end{aligned}
$$

Proof. By Lemma 4.10, we have

$$
\begin{aligned}
& F\left(\sigma_{12}\left(\mathcal{Y}_{a_{3} a_{2} ; r}^{a_{2} ;(6)}\right) \otimes \sigma_{12}\left(\mathcal{Y}_{a_{2} a_{2}^{\prime} ; q}^{a_{3} ;(2)}\right) ; \mathcal{Y}_{e a_{2} ; 1}^{a_{2}} \otimes \mathcal{Y}_{a_{2} a_{2}^{\prime} ; 1}^{e}\right) \\
& =\left\langle\sigma_{132}\left(\mathcal{Y}_{a_{3} a_{2} ; r}^{a_{2} ;(6)}\right), \sigma_{12}\left(\mathcal{Y}_{a_{2} a_{2}^{\prime} ; q^{\prime}}^{a_{3} ;(2)}\right\rangle_{\mathcal{V}_{a_{2} a_{2}^{\prime}}^{a^{\prime}}}\right. \\
& =\frac{\sqrt{F_{a_{2}}} \sqrt{F_{a_{2}^{\prime}}}}{\sqrt{F_{a_{3}^{\prime}}}}\left(\sigma_{132}\left(\mathcal{Y}_{a_{3} a_{2} ; r}^{a_{2} ;(6)}\right), \sigma_{12}\left(\mathcal{Y}_{a_{2} a_{2}^{\prime} ; q}^{a_{3}^{\prime} ;(2)}\right)_{\mathcal{V}_{a_{2} a_{2}^{\prime}}^{a_{3}^{\prime}}}\right. \\
& =\frac{\sqrt{F_{a_{2}}} \sqrt{F_{a_{2}^{\prime}}}}{\sqrt{F_{a_{3}^{\prime}}}}\left(\sigma_{13}\left(\mathcal{Y}_{a_{3} a_{2} ; r}^{a_{2} ;(6)}\right), \mathcal{Y}_{a_{2}^{\prime} a_{2} ; q}^{a_{3}^{\prime}(2)}\right)_{\mathcal{V}_{a_{2}^{\prime} a_{2}}^{a_{3}^{\prime}}} \\
& =\frac{\sqrt{F_{a_{2}}} \sqrt{F_{a_{2}^{\prime}}}}{\sqrt{F_{a_{3}^{\prime}}}}\left(\sigma_{13}\left(\mathcal{Y}_{a_{3} a_{2} ; r}^{a_{2} ;(6)}\right), \sigma_{13}\left(\mathcal{Y}_{a_{3}^{\prime} a_{2}^{\prime} ; a^{\prime} ;(6)}^{a^{\prime}}\right)\right)_{\mathcal{V}_{a_{2}^{\prime} a_{2}}^{a_{3}^{\prime}}} \\
& =\frac{\sqrt{F_{a_{2}}} \sqrt{F_{a_{2}^{\prime}}}}{\sqrt{F_{a_{3}^{\prime}}}}\left(\mathcal{Y}_{a_{3} a_{2} ; r}^{a_{2} ;(6)}, \mathcal{Y}_{a_{3}^{\prime} ; a_{2}^{\prime} ; q}^{\prime} ;\right)_{a_{3} a_{2}}^{a_{2}} \\
& =\frac{\sqrt{F_{a_{2}}} \sqrt{F_{a_{2}^{\prime}}}}{\sqrt{F_{a_{3}^{\prime}}}} \frac{\sqrt{F_{a_{2}}}}{\sqrt{F_{a_{3}}} \sqrt{F_{a_{2}}}}\left\langle\mathcal{Y}_{a_{3} a_{2} ; r}^{a_{2} ;(6)}, \mathcal{Y}_{a_{3}^{\prime} a_{2}^{\prime} ; q}^{\prime} ;\right\rangle_{\mathcal{V}_{3} a_{2}}^{a_{2}} \\
& =\frac{F_{a_{2}}}{F_{a_{3}}} \delta_{r q},
\end{aligned}
$$

where in the last step, we have used the fact that $F_{a}=F_{a^{\prime}}$ for $a \in \mathcal{A}$. Using Lemma 4.9, (4.25), the formula $S_{e}^{a_{1}}=\frac{S_{e}^{e}}{F_{a_{1}}}$ and (4.20), we obtain (4.24).

Lemma 4.12. For $a_{1}, a_{2}, a_{3} \in \mathcal{A}$, we have

$$
S\left(\sigma_{23}\left(\mathcal{Y}_{a_{3} a_{1} ; p}^{a_{1} ;(1)}\right) ; \sigma_{23}\left(\mathcal{Y}_{a_{3} a_{2} ; r}^{a_{2} ;(6)}\right)\right)=S\left(\mathcal{Y}_{a_{3} a_{1} ; p}^{a_{1} ;(1)} ; \mathcal{Y}_{a_{3} a_{2} ; r}^{a_{2} ;(6)}\right) .
$$


Proof. By (4.18) and the definition of $\Psi_{a_{1}, a_{3}}^{k}$, we have

$$
\begin{gathered}
\operatorname{Tr}_{W^{a_{1}}} \mathcal{Y}_{a_{3} a_{1} ; p}^{a_{1} ;(1)}\left(\mathcal{U}\left(e^{-2 \pi i \frac{z}{\tau}}\right)\left(-\frac{1}{\tau}\right)^{L(0)} w_{a_{3}}, e^{-2 \pi i \frac{z}{\tau}}\right) q_{-\frac{1}{\tau}}^{L(0)-\frac{c}{24}} \\
(4.27)=\sum_{a_{2} \in \mathcal{A}} \sum_{r=1}^{N_{a_{3} a_{2}}^{a_{2}}} S\left(\mathcal{Y}_{a_{3} a_{1} ; p}^{a_{1} ;(1)} ; \mathcal{Y}_{a_{3} a_{2} ; r}^{a_{2} ;(6)}\right) \operatorname{Tr}_{W^{a_{2}}} \mathcal{Y}_{a_{3} a_{2} ; r}^{a_{2} ;(6)}\left(\mathcal{U}\left(e^{2 \pi i z}\right) w_{a_{3}}, e^{2 \pi i z}\right) q_{\tau}^{L(0)-\frac{c}{24}} .
\end{gathered}
$$

On the other hand, we have

$$
\begin{aligned}
& \operatorname{Tr}_{W^{a_{1}}} \mathcal{Y}_{a_{3} a_{1} ; p}^{a_{1} ;(1)}\left(\mathcal{U}\left(e^{-2 \pi i \frac{z}{\tau}}\right)\left(-\frac{1}{\tau}\right)^{L(0)} w_{a_{3}}, e^{-2 \pi i \frac{z}{\tau}}\right) q_{-\frac{1}{\tau}}^{L(0)-\frac{c}{24}} \\
& =\operatorname{Tr}_{W^{a_{1}}} \sigma_{23}^{2}\left(\mathcal{Y}_{a_{3} a_{1} ; p}^{a_{1} ;(1)}\right)\left(\mathcal{U}\left(e^{-2 \pi i \frac{z}{\tau}}\right)\left(-\frac{1}{\tau}\right)^{L(0)} w_{a_{3}}, e^{-2 \pi i \frac{z}{\tau}}\right) q_{-\frac{1}{\tau}}^{L(0)-\frac{c}{24}} \\
& =\operatorname{Tr}_{\left(W^{a_{1}}\right)^{\prime}} \sigma_{23}\left(\mathcal{Y}_{a_{3} a_{1} ; p}^{a_{1} ;(1)}\right)\left(\mathcal{U}\left(e^{2 \pi i \frac{z}{\tau}}\right) e^{-\pi i L(0)}\left(-\frac{1}{\tau}\right)^{L(0)} w_{a_{3}}, e^{2 \pi i \frac{z}{\tau}}\right) q_{-\frac{1}{\tau}}^{L(0)-\frac{c}{24}} \\
& =\sum_{a_{2} \in \mathcal{A}} \sum_{r=1}^{N_{a_{3} a_{2}}^{a_{2}}} S\left(\sigma_{23}\left(\mathcal{Y}_{a_{3} a_{1} ; p}^{a_{1} ;(1)}\right) ; \sigma_{23}\left(\mathcal{Y}_{a_{3} a_{2} ; r}^{a_{2} ;(6)}\right)\right) \text {. } \\
& \cdot \operatorname{Tr}_{\left(W^{a_{2}}\right)^{\prime}} \sigma_{23}\left(\mathcal{Y}_{a_{3} a_{2} ; r}^{a_{2} ;(6)}\right)\left(\mathcal{U}\left(e^{-2 \pi i z}\right) e^{-\pi i L(0)} w_{a_{3}}, e^{-2 \pi i z}\right) q_{\tau}^{L(0)-\frac{c}{24}} \\
& =\sum_{a_{2} \in \mathcal{A}} \sum_{r=1}^{N_{a_{3} a_{2}}^{a_{2}}} S\left(\sigma_{23}\left(\mathcal{Y}_{a_{3} a_{1} ; p}^{a_{1} ;(1)}\right) ; \sigma_{23}\left(\mathcal{Y}_{a_{3} a_{2} ; r}^{a_{2} ;(6)}\right)\right) \text {. } \\
& \cdot \operatorname{Tr}_{W^{a_{2}}} \mathcal{Y}_{a_{3} a_{2} ; r}^{a_{2} ;(6)}\left(\mathcal{U}\left(e^{2 \pi i z}\right) w_{a_{3}}, e^{2 \pi i z}\right) q_{\tau}^{L(0)-\frac{c}{24}} .
\end{aligned}
$$

From (4.27) and (4.28), we obtain (4.26).

The following result is a generalization of Theorem 5.6 in [H10]:

Theorem 4.13. For $a_{1}, a_{2}, a_{3} \in \mathcal{A}$, we have

$$
S\left(\mathcal{Y}_{a_{3}^{\prime} a_{2}^{\prime} ; r}^{\prime a} ; \mathcal{Y}_{a_{3}^{\prime} a_{1}^{\prime} ; p}^{\prime ; a^{\prime} ;(1)}\right)=S\left(\mathcal{Y}_{a_{3} a_{1} ; p}^{a_{1} ;(1)} ; \mathcal{Y}_{a_{3} a_{2} ; r}^{a_{2} ;(6)}\right)
$$

Proof. From (4.24), we obtain

$$
\begin{aligned}
& S\left(\mathcal{Y}_{a_{3}^{\prime} a_{2}^{\prime} ; r}^{\prime a_{2}^{\prime} ;(6)} ; \mathcal{Y}_{a_{3}^{\prime} a_{1}^{\prime} ; p}^{\prime ; a_{1}^{\prime} ;(1)}\right)
\end{aligned}
$$

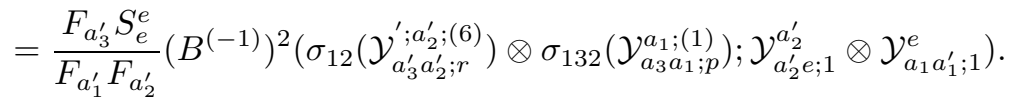

Using (3.1), (3.5), (3.12) in [H10, the relations $\sigma_{132}^{2}=\sigma_{123}=\sigma_{12} \sigma_{23}, \sigma_{123} \sigma_{12}=$ $\sigma_{132} \sigma_{23}, \sigma_{12} \sigma_{132} \sigma_{12}=\sigma_{123}, \sigma_{12} \sigma_{123} \sigma_{12}=\sigma_{132}$ and $h_{a}=h_{a^{\prime}}$ for $a \in \mathcal{A}$, we obtain

$$
\begin{aligned}
& \left(B^{(-1)}\right)^{2}\left(\sigma_{12}\left(\mathcal{Y}_{a_{3}^{\prime} a_{2}^{\prime} ; r}^{\left.\prime a_{2}^{\prime} ; 6\right)} \otimes \sigma_{132}\left(\mathcal{Y}_{a_{3} a_{1} ; p}^{a_{1} ;(1)}\right) ; \mathcal{Y}_{a_{2}^{\prime} e ; 1}^{a_{2}^{\prime}} \otimes \mathcal{Y}_{a_{1} a_{1}^{\prime} ; 1}^{e}\right)\right.
\end{aligned}
$$

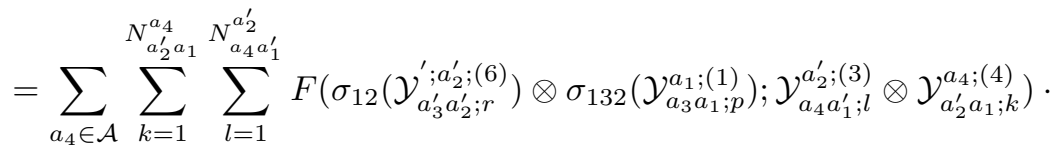




$$
\begin{aligned}
& \cdot e^{-2 \pi i\left(h_{a_{4}}-h_{a_{2}^{\prime}}-h_{a_{1}}\right)} F^{-1}\left(\mathcal{Y}_{a_{4} a_{1}^{\prime} ; l}^{a_{2}^{\prime} ;(3)} \otimes \mathcal{Y}_{a_{2}^{\prime} a_{1} ; k}^{a_{4} ;(4)} ; \mathcal{Y}_{a_{2}^{\prime} ; 1}^{a_{2}^{\prime}} \otimes \mathcal{Y}_{a_{1} a_{1}^{\prime} ; 1}^{e}\right) \\
& =\sum_{a_{4} \in \mathcal{A}} \sum_{k=1}^{N_{a_{2}^{\prime} a_{1}}^{a_{4}}} \sum_{l=1}^{N_{a_{4} a_{1}^{\prime}}^{a_{2}^{\prime}}} e^{-2 \pi i\left(h_{a_{4}}-h_{a_{2}^{\prime}}-h_{a_{1}}\right)} \text {. } \\
& \cdot F\left(\sigma_{132}^{2}\left(\mathcal{Y}_{a_{3} a_{1} ; p}^{a_{1} ;(1)}\right) \otimes \sigma_{123}\left(\sigma_{12}\left(\mathcal{Y}_{a_{3}^{\prime} a_{2}^{\prime} ; r}^{\prime ; a_{2}^{\prime} ;(6)}\right)\right) ; \sigma_{123}\left(\mathcal{Y}_{a_{2}^{\prime} a_{1} ; k}^{a_{4} ;(4)}\right) \otimes \sigma_{132}\left(\mathcal{Y}_{a_{4} a_{1}^{\prime} ; l}^{a_{2}^{\prime} ;(3)}\right)\right) . \\
& \cdot F\left(\sigma_{12}\left(\mathcal{Y}_{a_{4} a_{1}^{\prime} ; l}^{a_{2}^{\prime} ;(3)}\right) \otimes \sigma_{12}\left(\mathcal{Y}_{a_{2}^{\prime} a_{1} ; k}^{a_{4} ;(4)}\right) ; \sigma_{12}\left(\mathcal{Y}_{a_{2}^{\prime} ; 1}^{a_{2}^{\prime}}\right) \otimes \sigma_{12}\left(\mathcal{Y}_{a_{1} a_{1}^{\prime} ; 1}^{e}\right)\right) \\
& =\sum_{a_{4} \in \mathcal{A}} \sum_{k=1}^{N_{a_{2}^{\prime} a_{1}}^{a_{4}}} \sum_{l=1}^{N_{a_{4} a_{1}^{\prime}}^{a^{\prime}}} e^{-2 \pi i\left(h_{a_{4}}-h_{a_{2}^{\prime}}-h_{a_{1}}\right)} \\
& \cdot F\left(\sigma_{132}^{2}\left(\mathcal{Y}_{a_{3} a_{1} ; p}^{a_{1} ;(1)}\right) \otimes \sigma_{123}\left(\sigma_{12}\left(\mathcal{Y}_{a_{3}^{\prime} a_{2}^{\prime} ; r}^{\prime} ; a_{2}^{\prime} ;(6)\right) ; \sigma_{123}\left(\mathcal{Y}_{a_{2}^{\prime} a_{1} ; k}^{a_{4} ;(4)}\right) \otimes \sigma_{132}\left(\mathcal{Y}_{a_{4} a_{1}^{\prime} ; l}^{a_{2}^{\prime} ;(3)}\right)\right)\right. \text {. } \\
& \cdot F\left(\sigma _ { 1 3 2 } \left(\sigma_{12}\left(\mathcal{Y}_{a_{2}^{\prime} a_{1} ; k}^{\left.a_{4} ; 4\right)}\right) \otimes \sigma_{123}\left(\sigma_{12}\left(\mathcal{Y}_{a_{4} a_{1}^{\prime} ; l}^{a_{2}^{\prime} ;(3)}\right)\right)\right.\right. \\
& \left.\sigma_{123}\left(\sigma_{12}\left(\mathcal{Y}_{a_{1} a_{1}^{\prime} ; 1}^{e}\right)\right) \otimes \sigma_{132}\left(\sigma_{12}\left(\mathcal{Y}_{a_{2}^{\prime} e ; 1}^{a_{2}^{\prime}}\right)\right)\right) \\
& =\sum_{a_{4} \in \mathcal{A}} \sum_{k=1}^{N_{a_{2}^{\prime} a_{1}}^{a_{4}}} \sum_{l=1}^{N_{a_{4} a_{1}^{\prime}}^{a_{2}^{\prime}}} e^{-2 \pi i\left(h_{a_{4}}-h_{a_{2}^{\prime}}-h_{a_{1}}\right)} . \\
& \cdot F\left(\sigma_{132}^{2}\left(\mathcal{Y}_{a_{3} a_{1} ; p}^{a_{1} ;(1)}\right) \otimes \sigma_{123}\left(\sigma_{12}\left(\mathcal{Y}_{a_{3}^{\prime} a_{2}^{\prime} ; r}^{\prime} a^{\prime} ;(6)\right) ; \sigma_{123}\left(\mathcal{Y}_{a_{2}^{\prime} a_{1} ; k}^{a_{4} ;(4)}\right) \otimes \sigma_{132}\left(\mathcal{Y}_{a_{4} a_{1}^{\prime} ; l}^{a_{2}^{\prime} ;(3)}\right)\right)\right. \text {. } \\
& \cdot F^{-1}\left(\sigma _ { 1 2 } \left(\sigma _ { 1 3 2 } ( \sigma _ { 1 2 } ( \mathcal { Y } _ { a _ { 2 } ^ { \prime } a _ { 1 } ; k } ^ { a _ { 4 } ; 4 ) } ) ) \otimes \sigma _ { 1 2 } \left(\sigma_{123}\left(\sigma_{12}\left(\mathcal{Y}_{a_{4} a_{1}^{\prime} ; l}^{\left.a_{2}^{\prime} ; 3\right)}\right)\right)\right.\right.\right. \text {; } \\
& \left.\sigma_{12}\left(\sigma_{123}\left(\sigma_{12}\left(\mathcal{Y}_{a_{1} a_{1}^{\prime} ; 1}^{e}\right)\right)\right) \otimes \sigma_{12}\left(\sigma_{132}\left(\sigma_{12}\left(\mathcal{Y}_{a_{2}^{\prime} e ; 1}^{a_{2}^{\prime}}\right)\right)\right)\right)
\end{aligned}
$$

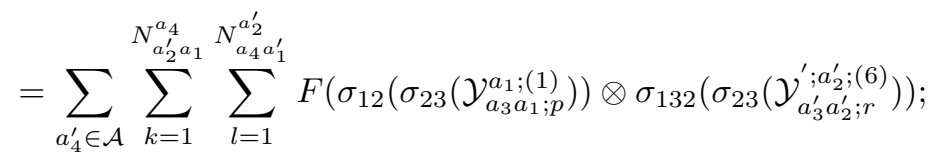

$$
\begin{aligned}
& \left.\sigma_{123}\left(\mathcal{Y}_{a_{2}^{\prime} a_{1} ; k}^{a_{4} ;()}\right) \otimes \sigma_{132}\left(\mathcal{Y}_{a_{4} a_{1}^{\prime} ; l}^{a_{2}^{\prime} ;(3)}\right)\right) \\
& \cdot e^{-2 \pi i\left(h_{a_{4}^{\prime}}-h_{a_{1}^{\prime}}-h_{a_{2}}\right)} \text {. } \\
& \cdot F^{-1}\left(\sigma_{123}\left(\mathcal{Y}_{a_{2}^{\prime} a_{1} ; k}^{a_{4} ;(4)}\right) \otimes \sigma_{132}\left(\mathcal{Y}_{a_{4} a_{1}^{\prime} ; l}^{a_{2}^{\prime} ;(3)}\right) ; \mathcal{Y}_{a_{1}^{\prime} e ; 1}^{a_{1}^{\prime}} \otimes \mathcal{Y}_{a_{2} a_{2}^{\prime} ; 1}^{e}\right) \\
& (4.31)=\left(B^{(-1)}\right)^{2}\left(\sigma_{12}\left(\sigma_{23}\left(\mathcal{Y}_{a_{3} a_{1} ; p}^{a_{1} ;(1)}\right)\right) \otimes \sigma_{132}\left(\sigma_{23}\left(\mathcal{Y}_{a_{3}^{\prime} a_{2}^{\prime} ; r}^{\prime} ; a_{2}^{\prime} ;(6)\right) ; \mathcal{Y}_{a_{1}^{\prime} \ell ; 1}^{a_{1}^{\prime}} \otimes \mathcal{Y}_{a_{2} a_{2}^{\prime} ; 1}^{e}\right)\right. \text {. }
\end{aligned}
$$

Substituting the right-hand side of (4.31) into the right-hand side of (4.30) and then using (4.24) and (4.26), we obtain

$$
\begin{aligned}
& S\left(\mathcal{Y}_{a_{3}^{\prime} a_{2}^{\prime} ; r}^{\prime} ; a^{\prime} ;(6) ; \mathcal{Y}_{a_{3}^{\prime} a_{1}^{\prime} ; p}^{\prime} a_{1}^{\prime} ;(1)\right. \\
& =\frac{F_{a_{3}^{\prime}} S_{e}^{e}}{F_{a_{1}^{\prime}} F_{a_{2}^{\prime}}}\left(B^{(-1)}\right)^{2}\left(\sigma_{12}\left(\sigma_{23}\left(\mathcal{Y}_{a_{3} a_{1} ; p}^{a_{1} ;(1)}\right)\right) \otimes \sigma_{132}\left(\sigma_{23}\left(\mathcal{Y}_{a_{3}^{\prime} a_{2}^{\prime} ; r}^{\prime} a_{2}^{\prime} ;(6)\right) ; \mathcal{Y}_{a_{1}^{\prime} ; 1}^{a_{1}^{\prime}} \otimes \mathcal{Y}_{a_{2} a_{2}^{\prime} ; 1}^{e}\right)\right. \\
& =S\left(\sigma_{23}\left(\mathcal{Y}_{a_{3} a_{1} ; p}^{a_{1} ;(1)}\right) ; \sigma_{23}\left(\mathcal{Y}_{a_{3} a_{2} ; r}^{a_{2} ;(6)}\right)\right) \\
& =S\left(\mathcal{Y}_{a_{3} a_{1} ; p}^{a_{1} ;(1)} ; \mathcal{Y}_{a_{3} a_{2} ; r}^{a_{2} ;(6)}\right) \text {. }
\end{aligned}
$$


We now prove the modular invariance of the conformal full field algebras over $V \otimes V$ constructed in $[\mathrm{HK}]$ (see Section 2) for $V$ satisfying the conditions in the preceding two sections.

Theorem 4.14. The conformal full field algebra over $V \otimes V$ given in Theorem 1.8 is modular invariant.

Proof. By Theorem 3.8, we need only verify (3.19). In this case, $\mathcal{A}^{L}=\mathcal{A}^{R}=\mathcal{A}$ and we can identify the set $\{1, \ldots, N\}$ with $\mathcal{A}$. The map $r^{L}$ as a map from $\mathcal{A}$ to $\mathcal{A}$ is the identity map and the map ${ }^{R}$ as a map from $\mathcal{A}$ to $\mathcal{A}$ is the map '. Using the basis of the intertwining operators that we choose in the construction of the conformal full field algebra over $V \otimes V$, for $a_{1}, a_{2} \in \mathcal{A}$, we have $d_{a_{1}, a_{2} ; i, j}^{\left.a_{2} ; 1,1\right)}=\delta_{i j}$. So in this case the left-hand side of (3.19) is equal to

$$
\begin{aligned}
\sum_{a_{1} \in \mathcal{A}} & \sum_{i=1}^{N_{a a_{1}}^{a_{1}}} \sum_{j=1}^{N_{a^{\prime} a_{1}^{\prime}}^{a_{1}^{\prime}}} \delta_{i j} S\left(\mathcal{Y}_{a a_{1} ; i}^{a_{1}} ; \mathcal{Y}_{a a_{2} ; k}^{a_{2}}\right) S^{-1}\left(\mathcal{Y}_{a^{\prime} a_{1}^{\prime} ; j}^{a_{1}^{\prime}} ; \mathcal{Y}_{a^{\prime} a_{3}^{\prime} ; l}^{a_{3}^{\prime}}\right) \\
= & \sum_{a_{1} \in \mathcal{A}} \sum_{i=1}^{N_{a a_{1}}^{a_{1}}} S\left(\mathcal{Y}_{a^{\prime} a_{2}^{\prime} ; k}^{a_{2}^{\prime}} ; \mathcal{Y}_{a^{\prime} a_{1}^{\prime} ; i}^{a_{1}^{\prime}}\right) S^{-1}\left(\mathcal{Y}_{a^{\prime} a_{1}^{\prime} ;}^{a_{1}^{\prime}} ; \mathcal{Y}_{a^{\prime} a_{3}^{\prime} ; l}^{a_{3}^{\prime}}\right) \\
= & \delta_{a_{2}^{\prime} a_{3}^{\prime}} \delta_{k l} \\
= & \sum_{a_{4} \in\left(r^{L}\right)^{-1}\left(a_{2}\right) \cap\left(r^{R}\right)^{-1}\left(a_{3}^{\prime}\right)} \delta_{k l},
\end{aligned}
$$

which is indeed equal to the right-hand side of (3.19). By Theorem 3.8, the conformal full field algebra over $V \otimes V$ is modular invariant.

\section{ACKNOWLEDGMENT}

The first author was partially supported by NSF grant DMS-0401302.

\section{REFERENCES}

[BK] B. Bakalov and A. Kirillov, Jr., Lectures on tensor categories and modular functors, University Lecture Series, Vol. 21, Amer. Math. Soc., Providence, RI, 2001. MR1797619 (2002d:18003)

[BPZ] A. A. Belavin, A. M. Polyakov and A. B. Zamolodchikov, Infinite conformal symmetries in two-dimensional quantum field theory, Nucl. Phys. B241 (1984), 333-380. MR757857 $(86 \mathrm{~m}: 81097)$

[B] R. Borcherds, Monstrous moonshine and monstrous Lie superalgebras, Invent. Math. 109 (1992), 405-444. MR 1172696 (94f:11030)

[FFFS] G. Felder, J. Fröhlich, J. Fuchs and C. Schweigert, Correlation functions and boundary conditions in rational conformal field theory and three-dimensional topology, Compositio Math. 131 (2002), 189-237. MR1898435 (2003e:57051)

[FHL] I. Frenkel, Y.-Z. Huang and J. Lepowsky, On axiomatic approaches to vertex operator algebras and modules, Memoirs Amer. Math. Soc. 104, 1993. MR1142494 (94a:17007)

[FLM] I. Frenkel, J. Lepowsky, and A. Meurman, Vertex operator algebras and the Monster, Pure and Appl. Math., Vol. 134, Academic Press, New York, 1988. MR996026 (90h:17026)

[FjFRS1] J. Fjelstad, J. Fuchs, I. Runkel and C. Schweigert, TFT construction of RCFT correlators V: Proof of modular invariance and factorisation, Theory and Applications of Categories 16 (2006), 342-433. MR2259258 (2008a:81199)

[FjFRS2] I. Runkel, J. Fjelstad, J. Fuchs and C. Schweigert, Topological and conformal field theory as Frobenius algebras, in: Proceedings of the Streetfest (Canberra, July, 2005), Contemp. Math. Vol. 431, Amer. Math. Soc., Providence, RI, 2007. MR2342831 
[FrFRS] J. Fröhlich, J. Fuchs, I. Runkel and C. Schweigert, Correspondences of ribbon categories, Adv. Math. 199 (2006), 192-329. MR2187404 (2007b:18007)

[FRS1] J. Fuchs, I. Runkel and C. Schweigert, Conformal correlation functions, Frobenius algebras and triangulations, Nucl. Phys. B624 (2002), 452-468. MR.1882479 (2002j:81215)

[FRS2] J. Fuchs, I. Runkel and C. Schweigert, TFT construction of RCFT correlators I: Partition functions, Nucl. Phys. B646 (2002), 353-497. MR.1940282(2004c:81244)

[FRS3] J. Fuchs, I. Runkel and C. Schweigert, TFT construction of RCFT correlators II: Unoriented world sheets Nucl.Phys. B678 (2004), 511-637. MR2026879(2005a:81197)

[FRS4] J. Fuchs, I. Runkel and C. Schweigert, TFT construction of RCFT correlators III: Simple currents Nucl.Phys. B694 (2004), 277-353. MR2076134(2005e:81209)

[FRS5] J. Fuchs, I. Runkel and C. Schweigert, TFT construction of RCFT correlators, IV: Structure constants and correlation functions, Nucl. Phys. B715 (2005), 539-638. MR2137114 (2006g:81161)

[H1] Y.-Z. Huang, A theory of tensor products for module categories for a vertex operator algebra, IV, J. Pure Appl. Alg. 100 (1995), 173-216. MR1344849(98a:17050)

[H2] Y.-Z. Huang, Virasoro vertex operator algebras, (nonmeromorphic) operator product expansion and the tensor product theory, J. Alg. 182 (1996), 201-234. MR1388864 (97h:17029)

[H3] Y.-Z. Huang, Intertwining operator algebras, genus-zero modular functors and genuszero conformal field theories, in: Operads: Proceedings of Renaissance Conferences, ed. J.-L. Loday, J. Stasheff, and A. A. Voronov, Contemporary Math., Vol. 202, Amer. Math. Soc., Providence, 1997, 335-355. MR1436926 (98a:17051)

[H4] Y.-Z. Huang, Genus-zero modular functors and intertwining operator algebras, Internat. J. Math. 9 (1998), 845-863. MR.1651049 (99i:17031)

[H5] Y.-Z. Huang, Generalized rationality and a "Jacobi identity" for intertwining operator algebras, Selecta Math. (N.S.), 6 (2000), 225-267. MR1817614 (2002c:17041)

[H6] Y.-Z. Huang, Differential equations and intertwining operators, Comm. Contemp. Math. 7 (2005), 375-400. MR2151865 (2006e:17037)

[H7] Y.-Z. Huang, Differential equations, duality and modular invariance, Comm. Contemp. Math. 7 (2005), 649-706. MR2175093(2007a:11057)

[H8] Y.-Z. Huang, Vertex operator algebras, the Verlinde conjecture and modular tensor categories, Proc. Natl. Acad. Sci. USA 102 (2005), 5352-5356. MR2140309 (2006a:17026)

[H9] Y.-Z. Huang, Vertex operator algebras, fusion rules and modular transformations, in: Noncommutative Geometry and Representation Theory in Mathematical Physics, ed. J. Fuchs, J. Mickelsson, G. Rozenblioum and A. Stolin, Contemporary Math. Vol. 391, Amer. Math. Soc., Providence, 2005, 135-148. MR2184018 (2006j:17027)

[H10] Y.-Z. Huang, Vertex operator algebras and the Verlinde conjecture, Commun. Contemp. Math. 10 (2008), 103-154. MR2387861

[H11] Y.-Z. Huang, Rigidity and modularity of vertex tensor categories, Commun. Contemp. Math. 10 (2008), 871-911. MR2468370

[HK] Y.-Z. Huang and L. Kong, Full field algebras, Comm. Math. Phys. 272 (2007), 345-396. MR 2300247 (2008g:81102)

[KO] A. Kapustin and D. Orlov, Vertex algebras, mirror symmetry, and D-branes: The case of complex tori, Comm. Math. Phys. 233 (2003), 79-136. MR.1957733 (2004b:14073)

[Kong1] L. Kong, A mathematical study of open-closed conformal field theories, Ph.D. thesis, Rutgers University, 2005.

[Kong2] L. Kong, Full field algebras, operads and tensor categories, Adv. Math. 213 (2007), 271-340. MR2331245 (2008i:17036)

[Kont] M. Kontsevich, Rational conformal field theory and invariants of 3-dimensional manifolds, preprint CPT-88/P.2189, University of Marseille, 1988.

[MS1] G. Moore and N. Seiberg, Polynomial equations for rational conformal field theories, Phys. Lett. B212 (1988), 451-460. MR 962600 (89m:81155)

[MS2] G. Moore and N. Seiberg, Classical and quantum conformal field theory, Comm. Math. Phys. 123 (1989), 177-254. MR.1002038(90e:81216)

[MS3] G. Moore and N. Seiberg, Lectures on RCFT, in: Physics, geometry, and topology (Banff, AB, 1989), ed. H.C. Lee, NATO Adv. Sci. Inst. Ser. B Phys., 238, Plenum, New York, 1990, 263-361. MR1153682 (93m:81133b) 
[S1] G. Segal, The definition of conformal field theory, in: Differential geometrical methods in theoretical physics (Como, 1987), NATO Adv. Sci. Inst. Ser. C Math. Phys. Sci., 250, Kluwer Acad. Publ., Dordrecht, 1988, 165-171. MR981378 (90d:58026)

[S2] G. Segal, Two-dimensional conformal field theories and modular functors, in: Proceedings of the IXth International Congress on Mathematical Physics, Swansea, 1988, Hilger, Bristol, 1989, 22-37. MR.1033753 (92b:81192)

[S3] G. Segal, The definition of conformal field theory, preprint, 1988; also in: Topology, geometry and quantum field theory, ed. U. Tillmann, London Math. Soc. Lect. Note Ser., Vol. 308. Cambridge University Press, Cambridge, 2004, 421-577. MR2079383 (2005h:81334)

[Ts] H. Tsukada, String path integral realization of vertex operator algebras, Mem. Amer. Math. Soc. 91, 1991. MR.1052556 (91m:17044)

[Tu] V. G. Turaev, Quantum invariants of knots and 3-manifolds, de Gruyter Studies in Math., Vol. 18, Walter de Gruyter, Berlin, 1994. MR1292673 (95k:57014)

[V] E. Verlinde, Fusion rules and modular transformations in 2D conformal field theory, Nucl. Phys. B300 (1988), 360-376. MR.954762 (89h:81238)

[W] E. Witten, Three-dimensional gravity reconsidered, to appear; arXiv:0706.3359.

Department of Mathematics, Rutgers University, 110 Frelinghuysen Rd., Piscataway, NEW JERSEY 08854-8019

E-mail address: yzhuang@math.rutgers.edu

Max Planck Institute for Mathematics in the Sciences, Inselstrasse 22, D-04103, Leipzig, Germany - and - Institut Des Hautes Études Scientifiques, Le Bois-Marie, 35 , Route De Chartres, F-91440 Bures-Sur-Yvette, France

Current address: Institute for Advanced Study, Tsinghua University, Beijing 100084, People's Republic of China

E-mail address: kong.fan.liang@gmail.com 